\title{
ASYMPTOTIC ANALYSIS AND CONTROL OF A HYBRID SYSTEM COMPOSED BY TWO VIBRATING STRINGS CONNECTED BY A POINT MASS
}

\author{
C. CASTRO
}

\begin{abstract}
We consider a hybrid, one-dimensional, linear system consisting on two flexible strings connected by a point mass. It is known that this system presents two interesting features. First, it is well posed in an asymmetric space in which solutions have one more degree of regularity to one side of the point mass. Second, that the spectral gap vanishes asymptotically. We prove that the first property is a consequence of the second one. We also consider a system in which the point mass is replaced by a string of length $2 \varepsilon$ and density $1 / 2 \varepsilon$. We show that, as $\varepsilon \rightarrow 0$, the solutions of this system converge to those of the original one. We also analyze the convergence of the spectrum and obtain the well-posedness of the limit system in the asymmetric space as a consequence of non-standard uniform bounds of solutions of the approximate problems. Finally we consider the controllability problem. It is well known that the limit system with $L^{2}$-controls on one end is exactly controllable in an asymmetric space. We show how this result can be obtained as the limit when $\varepsilon \rightarrow 0$ of partial controllability results for the approximate systems in which the number of controlled frequencies converges to infinity as $\varepsilon \rightarrow 0$. This is done by means of some new results on non-harmonic Fourier series.
\end{abstract}

\section{INTRODUCTION}

In this paper we consider a linear hybrid system composed by two vibrating strings connected by a point mass. Assume that the strings occupy the intervals $(-1,0)$ and $(0,1)$ of the real line and are connected at $x=0$ by a point mass. Let us consider a function $u=u(x, t)$ describing the vertical displacements of the strings and denote by $z=z(t)$ the displacement of the point mass. Assuming that the strings are fixed at the extremes $x= \pm 1$, the equations modelling the vibrations of this hybrid system are as follows:

$$
\begin{cases}u_{t t}=u_{x x} & \text { for }-1<x<0, t>0, \\ u_{t t}=u_{x x} & \text { for } 0<x<1, t>0, \\ u\left(0^{+}, t\right)=u\left(0^{-}, t\right)=z(t) & \text { for } t>0 \\ M z_{t t}(t)=u_{x}\left(0^{+}, t\right)-u_{x}\left(0^{-}, t\right) & \text { for } t>0 \\ u(-1, t)=u(1, t)=0 & \text { for } t>0 .\end{cases}
$$

Departamento de Matemática Aplicada, Universidad Complutense, 28040 Madrid, Spain. E-mail: ccastro@sunma4.mat.ucm.es.

Received by the journal November 26, 1996. Revised January 3, 1997. Accepted for publication February 19, 1997.

This work was supported by grants PB93-1203 of the DGICYT (Spain) and SC1*CT91-0732 and CHRX-CT94-0471 of the European Union.

(C) Société de Mathématiques Appliquées et Industrielles. Typeset by AATEX. 
In (1.1) the first two equations are the one-dimensional wave equation. The third equation imposes the continuity of the three components of the mechanical system at $x=0$. The fourth equation describes the dynamics of the point mass. The parameter $M>0$ represents the mass concentrated at the point $x=0$. The last equation is due to the fixed end conditions at $x= \pm 1$. In these equations $v\left(0^{ \pm}\right)$denote the right and left lateral limits of the function $v$ at $x=0$.

REMARK 1.1. When $M=0$ we recover the continuity condition of $u_{x}$ at $x=$ 0 and the classical equations for the motion of a vibrating string occupying the interval $(-1,1)$ without point mass, i.e. with unit constant density everywhere in $(-1,1)$.

In the sequel, to simplify the notation we will assume that $M=1$. This system was studied by S. Hansen and E. Zuazua [4] from a control theoretical point of view. It was observed that the system is well posed in an asymmetric space in which solutions have one more degree of regularity to one side of the point mass. It was conjectured that this phenomenon is due to the lack of spectral gap that the presence of the point mass produces on the spectrum of the system. In this paper we prove that this is indeed true and we explain this singular phenomenon by means of an asymptotic analysis which consists, roughly, on viewing system (1.1) as the limit as $\varepsilon \rightarrow 0$ of a system connecting three strings occupying the intervals $(-1,-\varepsilon),(-\varepsilon, \varepsilon)$ and $(\varepsilon, 1)$, the middle one having a density of the order of $1 / 2 \varepsilon$. In this case the equations of motion are as follows:

$$
\left\{\begin{array}{lll}
u_{t t}=u_{x x}, & \text { for }-1<x<-\varepsilon, t>0 \\
\frac{1}{2 \varepsilon} u_{t t}=u_{x x}, & \text { for }-\varepsilon<x<\varepsilon, \quad t>0 \\
u_{t t}=u_{x x}, & \text { for } \varepsilon<x<1, \quad t>0 \\
u\left( \pm \varepsilon^{-}, t\right)=u\left( \pm \varepsilon^{+}, t\right), & \text { for } t>0 \\
u_{x}\left( \pm \varepsilon^{-}, t\right)=u_{x}\left( \pm \varepsilon^{+}, t\right), & \text { for } t>0 \\
u(-1, t)=u(1, t)=0, & \text { for } t>0 .
\end{array}\right.
$$

System (1.2) is well posed in the energy space

$$
X=H_{0}^{1}(-1,1) \times L^{2}(-1,1) .
$$

More precisely, for any $\left(u_{0}, u_{1}\right) \in X$ there exists an unique solution $u \in C\left([0, \infty) ; H_{0}^{1}(-1,1)\right) \cap C^{1}\left([0, \infty) ; L^{2}(-1,1)\right)$ of $(1.2)$ taking the initial data

$$
u(x, 0)=u_{0}(x), u_{t}(x, 0)=u_{1}(x) \text { in }(-1,1) .
$$

On the other hand, the energy

$$
\begin{aligned}
& E^{\varepsilon}(t)=\frac{1}{2} \int_{-1}^{-\varepsilon}\left[\left|u_{t}\right|^{2}+\left|u_{x}\right|^{2}\right] d x+\frac{1}{2} \int_{-\varepsilon}^{\varepsilon}\left[\frac{1}{2 \varepsilon}\left|u_{t}\right|^{2}+\left|u_{x}\right|^{2}\right] d x \\
& \quad+\frac{1}{2} \int_{\varepsilon}^{1}\left[\left|u_{t}\right|^{2}+\left|u_{x}\right|^{2}\right] d x
\end{aligned}
$$

remains constant in time.

REMARK 1.2. System (1.2) can also be viewed as the equations of motion of a string of density $1+\frac{1}{2 \varepsilon} \chi_{(-\varepsilon, \varepsilon)}, \chi_{(-\varepsilon, \varepsilon)}$ being the characteristic function of the interval $(-\varepsilon, \varepsilon)$. 
However, it is easy to see that sytem (1.2) is not well-posed in asymmetric spaces in which solutions have one more degree of regularity to one side of $x=0$. System (1.1) is well posed in the Hilbert space $Y=X \times \mathbb{R}$ in the sense that for any $\left(u_{0}, u_{1}, z_{1}\right) \in Y$ there exists an unique solution $(u, z) \in\left(C\left([0, \infty) ; H_{0}^{1}(-1,1)\right) \cap C^{1}\left([0, \infty) ; L^{2}(-1,1)\right), C^{1}([0, \infty))\right)$ of $(1.1)$ taking the initial data

$$
\left\{\begin{array}{l}
u(x, 0)=u_{0}(x), u_{t}(x, 0)=u_{1}(x) \quad \text { in }(-1,1) \\
z(0)=u_{0}(0), z_{t}(0)=z_{1} .
\end{array}\right.
$$

On the other hand, the energy

$$
E(t)=\frac{1}{2} \int_{-1}^{1}\left[\left|u_{t}(x, t)\right|^{2}+\left|u_{x}(x, t)\right|^{2}\right] d x+\frac{1}{2}\left|z_{t}(t)\right|^{2}
$$

remains constant for every solution of (1.1).

However, as we have said above, system (1.1) is also well-posed in an asymmetric space in which solutions have one more degree of regularity to one side of the point mass. For instance, this holds in the space of finiteenergy solutions such that their restriction to $(-1,0)$ belongs to $H^{2}(-1,0) \times$ $H^{1}(-1,1)$ and satisfying some further compatibility conditions.

In [4] the spectrum of (1.1) was analyzed and it was seen that the distance between consecutive eigenvalues tends to zero as the frequency increases. In this paper we analyze the spectrum of the approximate system (1.2). It is shown that its spectral gap is of the order of $\sqrt{\varepsilon}$. This provides an explanation of the fact that the spectral gap vanishes in the limit.

Classical results in spectral theory prove that the spectrum of (1.2) converges to the spectrum of (1.1) in the sense that the $k$-th eigen pair depends continuously on $\varepsilon$ even at $\varepsilon=0$. However, in order to explain the wellposedness of (1.1) we need some uniform convergence results for high frequencies too. Using classical asymptotic methods we show that, roughly, the eigenpairs converge uniformly with a rate of the order $\sqrt{\varepsilon}$ and for all the frequencies $k \leq \varepsilon^{-1 / 6}$. This uniform convergence result allows us to obtain all the singular phenomena related to the limit system when $\varepsilon \rightarrow 0$ from some uniform properties of the approximated systems.

For the approximate system (1.2) we construct finite-dimensional Fourier asymmetric spaces involving the frequencies $k \leq \varepsilon^{-1 / 6}$ in which systems (1.2) are uniformly well-posed. Passing to the limit as $\varepsilon \rightarrow 0$ we obtain the well-posedness of system (1.1) in an asymmetric Fourier space that we characterize as being constituted by finite-energy functions having one more degree of regularity to one side of the point mass.

Finally, we address the problem of controllability. In [4] it was shown that, if we act on system (1.1) by means of one $L^{2}$-control we may control exactly the initial data being, roughly, in $L^{2} \times H^{-1}$ to the right of $x=0$ and $H^{1} \times L^{2}$ to the left. The controllability space is therefore smaller than in the approximate systems (1.2) (in that case the space of controllable data is $\left.L^{2}(-1,1) \times H^{-1}(-1,1)\right)$ and differs by one degree of regularity in $(-1,0)$. As conjectured in [4] this fact can also be interpreted in terms of the vanishing of the spectral gap with the aid of the results by [12] on nonharmonic Fourier series. We prove a new result in this context showing that the results of [12] are stable under small perturbations of the spectrum. This 
allows us to prove that in the approximate system the frequencies $k \leq \varepsilon^{-1 / 6}$ of the asymmetric space are uniformly controllable. This shows that the controllability result of the limit system can be obtained as the limit when $\varepsilon \rightarrow 0$ of some uniform controllability property of the approximate systems.

In Remark 8.9 of section 8.3 we formulate an interesting open problem that, in our opinion, needs significant progresses in the theory of nonharmonic Fourier series. The question is roughly, whether the controls are uniformly bounded in $L^{2}(0, T)$ if the initial data are uniformly bounded in the asymmetric norms $\|\cdot\|_{\varepsilon}$ defined in (8.22) without the restrictions of having zero Fourier coefficients for the frequencies $|k| \geq K(\varepsilon)$.

The rest of the paper is organized as follows:

In section 2, we show how solutions of (1.1) can be obtained as limit of solutions of $(1.2)$ when $\varepsilon \rightarrow 0$. In section 3 we analyze the eigenvalue problems corresponding to (1.1) and (1.2). In particular, we show how the spectral gap associated to (1.2) is of the order of $\sqrt{\varepsilon}$ and tends to zero as $\varepsilon \rightarrow 0$. In section 4 we prove the convergence of the eigenvalues of (1.2) to those of (1.1). First we obtain some rough estimates by means of Rayleigh quotients. Later on we obtain precise convergence results by means of a careful asymptotic analysis. In section 5 we introduce an asymmetric space for system (1.1) in terms of Fourier series and we show how the wellposedness of (1.1) on it can be proved. In section 6 we recover this result as the limit when $\varepsilon \rightarrow 0$ of non-standard uniform estimates of solutions of the approximate system (1.2). In section 7 we obtain some further convergence results of solutions of (1.2) towards the solutions of (1.1). Finally, in section 8 we address to the control problem. First we recall the controllability result of [4] for the limit system (1.1). Then we obtain it as the limit as $\varepsilon \rightarrow 0$ of uniform partial controllability results for solutions of (1.2) in which the number of controlled frequencies converges to infinity as $\varepsilon \rightarrow 0$. This requires the obtention of some new results in the theory of non-harmonic Fourier series.

\section{Convergence of solutions}

Let us consider a family $\left\{u^{\varepsilon}\right\}_{\varepsilon>0}$ of solutions of (1.2) corresponding to the set of initial data $\left\{\left(u_{0}^{\varepsilon}, u_{1}^{\varepsilon}\right)\right\}_{\varepsilon>0}$ in $H_{0}^{1}(-1,1) \times L^{2}(-1,1)$. Assume that

$$
\left\{\begin{array}{c}
\left(u_{0}^{\varepsilon}, u_{1}^{\varepsilon}\right) \text { is bounded in } H_{0}^{1}(-1,1) \times L^{2}(-1,1), \\
\int_{-\varepsilon}^{\varepsilon}\left|u_{1}^{\varepsilon}\right|^{2} d x \leq C \varepsilon, \text { for some } C>0 .
\end{array}\right.
$$

Under these conditions, and as a consequence of the conservation of the energies $E^{\varepsilon}$ in (1.5) we deduce that the family of solutions $u^{\varepsilon}$ is bounded in

$$
\mathcal{Y}=L^{\infty}\left(0, \infty ; H_{0}^{1}(-1,1)\right) \cap W^{1, \infty}\left(0, \infty ; L^{2}(-1,1)\right) .
$$

We have the following weak convergence result:

THEOREM 2.1. Let us further assume that

$$
\left\{\begin{array}{l}
\left(u_{0}^{\varepsilon}, u_{1}^{\varepsilon}\right) \rightarrow\left(u_{0}, u_{1}\right) \text { weakly in } H_{0}^{1}(-1,1) \times L^{2}(-1,1) \text { as } \varepsilon \rightarrow 0 \\
\frac{1}{2 \varepsilon} \int_{-\varepsilon}^{\varepsilon} u_{1}^{\varepsilon}(x) d x \rightarrow z_{1} \text { as } \varepsilon \rightarrow 0
\end{array}\right.
$$


Then, the family $u^{\varepsilon}$ of solutions of (1.2) satisfies

$$
u^{\varepsilon} \rightarrow u \text { weakly }-* \text { in } \mathcal{Y}
$$

and

$$
\frac{1}{2 \varepsilon} \int_{-\varepsilon}^{\varepsilon} u_{t}^{\varepsilon}(x, t) d x \rightarrow u_{t}(0, t) \text { weakly }-* \text { in } L^{\infty}(0, \infty)
$$

where $u$ is the solution of the limit problem (1.1), (1.6) with initial data $\left(u_{0}, u_{1}, z_{1}\right)$.

REMARK 2.2. In view of the second condition in (2.1) we deduce that the quantities $\frac{1}{2 \varepsilon} \int_{-\varepsilon}^{\varepsilon} u_{1}^{\varepsilon}(x) d x$ are bounded. Indeed,

$$
\begin{aligned}
\left|\frac{1}{2 \varepsilon} \int_{-\varepsilon}^{\varepsilon} u_{1}^{\varepsilon}(x) d x\right| & \leq \frac{1}{2 \varepsilon} \int_{-\varepsilon}^{\varepsilon}\left|u_{1}^{\varepsilon}(x)\right| d x \leq \frac{1}{2 \varepsilon}\left(\int_{-\varepsilon}^{\varepsilon}\left|u_{1}^{\varepsilon}(x)\right|^{2} d x\right)^{\frac{1}{2}}(2 \varepsilon)^{1 / 2} \\
& =\left(\frac{1}{2 \varepsilon} \int_{-\varepsilon}^{\varepsilon}\left|u_{1}^{\varepsilon}(x)\right|^{2} d x\right)^{1 / 2} .
\end{aligned}
$$

To pass to the limit in (1.2) it is natural to assume that the limit of these quantities exists. This limit provides the initial velocity of the point mass in the limit system.

Proof. As we said above, the conservation of the energies provides an uniform bound for the solutions $u^{\varepsilon}$ in $\mathcal{Y}$. By extracting subsequences (that we still denote by the index $\varepsilon$ to simplify the notation) we have

$$
u^{\varepsilon} \rightarrow u \text { weakly }-* \text { in } \mathcal{Y} \text {. }
$$

Let us see that the limit $u$ satisfies (1.1).

Solutions of (1.2) are characterized by the following weak formulation:

$$
\left\{\begin{aligned}
\int_{0}^{\infty} & \int_{-1}^{-\varepsilon} u_{t}^{\varepsilon} \phi_{t} d x d t+\int_{0}^{\infty} \int_{\varepsilon}^{1} u_{t}^{\varepsilon} \phi_{t} d x d t+\frac{1}{2 \varepsilon} \int_{0}^{\infty} \int_{-\varepsilon}^{\varepsilon} u_{t}^{\varepsilon} \phi_{t} d x d t \\
& -\int_{0}^{\infty} \int_{-1}^{1} u_{x}^{\varepsilon} \phi_{x} d x d t+\int_{-1}^{\varepsilon} u_{1}^{\varepsilon} \phi(x, 0) d x+\frac{1}{2 \varepsilon} \int_{-\varepsilon}^{\varepsilon} u_{1}^{\varepsilon} \phi(x, 0) d x \\
& +\int_{\varepsilon}^{1} u_{1}^{\varepsilon} \phi(x, 0) d x=0, \quad \forall \phi \in C_{0}^{1}((-1,1) \times[0, \infty))
\end{aligned}\right.
$$

and the further initial condition

$$
u^{\varepsilon}(x, 0)=u_{0}^{\varepsilon}(x) \text { in }(-1,1) .
$$

The embedding from $\mathcal{Y}$ into $C\left([0, T] ; L^{2}(-1,1)\right)$ is compact for any $0<$ $T<\infty$. Therefore, passing to the limit in (2.8), the limit $u$ must satisfy the initial condition

$$
u(x, 0)=u_{0}(x) \text { in }(-1,1) .
$$

In order to pass to the limit in $(2.7)$ the only term that requires some careful analysis is

$$
I_{\varepsilon}=\frac{1}{2 \varepsilon} \int_{0}^{\infty} \int_{-\varepsilon}^{\varepsilon} u_{t}^{\varepsilon} \phi_{t} d x d t
$$


First we observe that

$$
\frac{1}{2 \varepsilon} \int_{-\varepsilon}^{\varepsilon} u_{t}^{\varepsilon}(x, t) d x \text { is bounded in } L^{\infty}(0, \infty) .
$$

Indeed, arguying as in Remark 2.2 we see that

$$
\left|\frac{1}{2 \varepsilon} \int_{-\varepsilon}^{\varepsilon} u_{t}^{\varepsilon}(x, t) d x\right| \leq\left[\frac{1}{2 \varepsilon} \int_{-\varepsilon}^{\varepsilon}\left|u_{t}^{\varepsilon}(x, t)\right|^{2} d x\right]^{1 / 2}
$$

and the latter is bounded by the conservation of the energies $E^{\varepsilon}$. Therefore, by extracting subsequences (that we still denote by the index $\varepsilon$ ), we deduce that

$$
\frac{1}{2 \varepsilon} \int_{-\varepsilon}^{\varepsilon} u_{t}^{\varepsilon}(x, t) d x \rightarrow w \text { weakly }-* \text { in } L^{\infty}(0, \infty)
$$

Let us see that $w(t)=u_{t}(0, t)$. For any $f \in C_{0}^{1}(0, \infty)$ we have

$$
\int_{0}^{\infty} \frac{1}{2 \varepsilon} \int_{-\varepsilon}^{\varepsilon} u_{t}^{\varepsilon}(x, t) d x f(t) d t=-\int_{0}^{\infty} \frac{1}{2 \varepsilon} \int_{-\varepsilon}^{\varepsilon} u^{\varepsilon}(x, t) f^{\prime}(t) d x d t
$$

The embedding from $\mathcal{Y}$ into $C([-1,1] \times[0, T])$ is compact for any $0<T<$ $\infty$. Therefore, in view of (2.6) the right hand side in (2.12) converges. More precisely,

$-\int_{0}^{\infty} \frac{1}{2 \varepsilon} \int_{-\varepsilon}^{\varepsilon} u^{\varepsilon}(x, t) f^{\prime}(t) d x d t \rightarrow-\int_{0}^{\infty} u(0, t) f^{\prime}(t) d t=\int_{0}^{\infty} u_{t}(0, t) f(t) d t$.

This shows that $w(t)=u_{t}(0, t)$ and concludes the proof of $(2.5)$.

Let us go back to the term $I_{\varepsilon}$. We claim that

$$
I_{\varepsilon}=\frac{1}{2 \varepsilon} \int_{0}^{\infty} \int_{-\varepsilon}^{\varepsilon} u_{t}^{\varepsilon} \phi_{t} d x d t \rightarrow \int_{0}^{\infty} u_{t}(0, t) \phi_{t}(0, t) d t .
$$

Indeed,

$$
\begin{aligned}
& \left|\frac{1}{2 \varepsilon} \int_{0}^{\infty} \int_{-\varepsilon}^{\varepsilon} u_{t}^{\varepsilon} \phi_{t} d x d t-\int_{0}^{\infty} u_{t}(0, t) \phi_{t}(0, t) d t\right| \\
& \leq\left|\int_{0}^{\infty} \frac{1}{2 \varepsilon} \int_{-\varepsilon}^{\varepsilon} u_{t}^{\varepsilon}(x, t)\left(\phi_{t}(x, t)-\phi_{t}(0, t)\right) d x d t\right| \\
& \quad+\left|\int_{0}^{\infty} \frac{1}{2 \varepsilon} \int_{-\varepsilon}^{\varepsilon}\left(u_{t}^{\varepsilon}-u_{t}(0, t)\right) \phi_{t}(0, t) d x d t\right|
\end{aligned}
$$

By (2.5) the last term converges to zero. For the other one we have:

$$
\begin{aligned}
& \left|\int_{0}^{\infty} \frac{1}{2 \varepsilon} \int_{-\varepsilon}^{\varepsilon} u_{t}^{\varepsilon}(x, t)\left(\phi_{t}(x, t)-\phi_{t}(0, t)\right) d x d t\right| \\
& \quad \leq \int_{0}^{\infty}\left(\frac{1}{2 \varepsilon} \int_{-\varepsilon}^{\varepsilon}\left|u_{t}^{\varepsilon}(x, t)\right|^{2} d x\right)^{1 / 2}\left(\frac{1}{2 \varepsilon} \int_{-\varepsilon}^{\varepsilon}\left(\phi_{t}(x, t)-\phi_{t}(0, t)\right)^{2} d x\right)^{1 / 2} d t \\
& \quad \leq\left(2 E^{\varepsilon}(0)\right)^{1 / 2} \int_{0}^{\infty}\left(\frac{1}{2 \varepsilon} \int_{-\varepsilon}^{\varepsilon}\left(\phi_{t}(x, t)-\phi_{t}(0, t)\right)^{2} d x\right)^{1 / 2} d t
\end{aligned}
$$

The initial energies $E^{\varepsilon}(0)$ are bounded and the last term in this inequality converges to zero since $\phi \in C^{1}$. This concludes the proof of (2.13).

Esaim: CoCV, July 1997, Vol.2, PP. 231-280 
We can now pass to the limit in (2.7). We easily get that the limit $u$, in addition to (2.9), satisfies

$$
\left\{\begin{aligned}
\int_{0}^{\infty} & \int_{-1}^{1}\left[u_{t} \phi_{t}-u_{x} \phi_{x}\right] d x d t+\int_{0}^{\infty} u_{t}(0, t) \phi_{t}(0, t) d t \\
& +\int_{-1}^{1} u_{1} \phi(x, 0) d x+z_{1} \phi(0,0)=0, \forall \phi \in C_{0}^{1}((-1,1) \times[0, \infty)) .
\end{aligned}\right.
$$

The variational equation (2.14) with (2.9) characterizes the solution of the limit system (1.1), (1.6) with initial data $\left(u_{0}, u_{1}, z_{1}\right)$. The solution $u$ of (1.1), (1.6) being unique we deduce that the whole family $u^{\varepsilon}$ converges as $\varepsilon \rightarrow 0$. This concludes the proof of Theorem 2.1.

The following theorem provides a strong convergence result:

Theorem 2.3. Let us assume that the initial data are such that

$$
\left\{\begin{array}{c}
\left(u_{0}^{\varepsilon}, u_{1}^{\varepsilon}\right) \rightarrow\left(u_{0}, u_{1}\right) \text { strongly in } H_{0}^{1}(-1,1) \times L^{2}(-1,1), \text { as } \varepsilon \rightarrow 0 \\
\frac{1}{2 \varepsilon} \int_{-\varepsilon}^{\varepsilon}\left|u_{1}^{\varepsilon}(x)\right|^{2} d x \rightarrow z_{1}^{2} \text {, as } \varepsilon \rightarrow 0 .
\end{array}\right.
$$

Then, the solutions $u^{\varepsilon}$ of (1.2) with data $\left(u_{0}^{\varepsilon}, u_{1}^{\varepsilon}\right)$ satisfy

$$
\left\{\begin{array}{c}
u^{\varepsilon} \rightarrow u \text { strongly in } H^{1}((-1,1) \times(0, T)) \text {, as } \varepsilon \rightarrow 0 \\
\frac{1}{2 \varepsilon} \int_{-\varepsilon}^{\varepsilon} u_{t}^{\varepsilon} d x \rightarrow u_{t}(0, t)=z^{\prime}(t) \text { strongly in } L^{2}(0, T) \text {, as } \varepsilon \rightarrow 0
\end{array}\right.
$$

for every $0<T<\infty$, where $u$ is the solution of (1.1), (1.6) with data $\left(u_{0}, u_{1}, z_{1}\right)$.

Proof. From Theorem 2.1 we know that convergences (2.15) hold in the weak topologies.

On the other hand, from (2.15) the initial energies $E^{\varepsilon}(0)$ converge to $E(0)$ as $\varepsilon \rightarrow 0$, and in view of the conservation of energies we deduce that $E^{\varepsilon}(t) \rightarrow E(t)$ for all $t>0$. Furthermore, by the dominated convergence Theorem,

$$
E^{\varepsilon} \rightarrow E \text { in } L^{2}(0, T)
$$

The strong convergences (2.16) are a direct consequence of the weak convergence and the convergence of the energies (2.17).

REMARK 2.4. In section 7 we will show that the strong convergence holds uniformly in time, i.e. in the space $\mathcal{Y}$.

\section{SPECTRAL ANALYSIS}

In this section we describe the main properties of the spectra of systems (1.1) and (1.2). First we analyze the approximate system (1.2) and then the limit system (1.1). 


\subsection{The approximate System}

The spectrum of system (1.2) consists of the positive real numbers $\nu>0$ such that the equation

$$
\left\{\begin{array}{l}
-u^{\prime \prime}=\nu\left(1+\frac{1}{2 \varepsilon} \chi_{(-\varepsilon, \varepsilon)}\right) u \quad \text { in }(-1,1) \\
u(-1)=u(1)=0
\end{array}\right.
$$

admits a non-trivial solution. Here and in the sequel' denotes derivation with respect to $x$.

The weak formulation of (3.1) is as follows:

$$
\left\{\begin{array}{l}
u \in H_{0}^{1}(-1,1) \\
\int_{-1}^{1} u^{\prime} v^{\prime} d x=\nu\left[\int_{-1}^{-\varepsilon} u v d x+\frac{1}{2 \varepsilon} \int_{-\varepsilon}^{\varepsilon} u v d x+\int_{\varepsilon}^{1} u v d x\right], \forall v \in H_{0}^{1}(-1,1) .
\end{array}\right.
$$

It is easy to see that $\nu$ is an eigenvalue of (3.1) if and only if $\nu=\frac{1}{\mu}, \mu$ being an eigenvalue of the compact, self-adjoint operator $T_{\varepsilon}: H_{0}^{1}(-1,1) \rightarrow$ $H_{0}^{1}(-1,1)$ such that $T_{\varepsilon} f=u$, where $u$ is the unique solution of the problem

$$
\left\{\begin{array}{l}
u \in H_{0}^{1}(-1,1) \\
\int_{-1}^{1} u^{\prime} v^{\prime} d x=\int_{-1}^{1}\left(1+\frac{1}{2 \varepsilon} \chi_{(-\varepsilon, \varepsilon)}\right) \text { fvdx } \quad \forall v \in H_{0}^{1}(-1,1) .
\end{array}\right.
$$

We deduce that the eigenvalues $\left\{\nu_{k}^{\varepsilon}\right\}_{k \geq 1}$ constitute an increasing sequence of distinct positive numbers

$$
0<\nu_{1}^{\varepsilon}<\nu_{2}^{\varepsilon}<\cdots<\nu_{k}^{\varepsilon}<\cdots
$$

and that the corresponding eigenfunctions $\left\{\phi_{k}^{\varepsilon}\right\}_{k \geq 1}$ form an orthonormal basis of $H_{0}^{1}(-1,1)$ for every $\varepsilon>0$.

Concerning the spectral gap we have the following:

Theorem 3.1. There exist positive constants $C_{i}>0, i=1,2$ such that

$$
C_{2} \sqrt{\varepsilon} \geq \inf _{j \neq k}\left|\sqrt{\nu_{j}^{\varepsilon}}-\sqrt{\nu_{k}^{\varepsilon}}\right| \geq C_{1} \sqrt{\varepsilon}
$$

for every $0<\varepsilon<1$.

Proof. First of all we prove the lower bound on the spectral gap, i.e.

$$
C_{1} \sqrt{\varepsilon} \leq \inf _{j \neq k}\left|\sqrt{\nu_{j}^{\varepsilon}}-\sqrt{\nu_{k}^{\varepsilon}}\right| .
$$

It is easy to see that the eigenfunctions of (3.1) are either even or odd functions. We start by considering the even eigenfunctions. In this case system (3.1) reduces to

$$
\left\{\begin{array}{l}
-u^{\prime \prime}=\nu\left(1+\frac{1}{2 \varepsilon} \chi_{(-\varepsilon, 0)}\right) u \quad \text { in }(-1,0) \\
u(-1)=u^{\prime}(0)=0 .
\end{array}\right.
$$

An easy computation shows that these eigenvalues are the roots of

$$
\frac{1}{\sqrt{2 \varepsilon}} \operatorname{tg}\left(\sqrt{\frac{\varepsilon \nu}{2}}\right)=\operatorname{cotg}(\sqrt{\nu}(1-\varepsilon)) \text {. }
$$

To simplify this equation we perform the change of variables

$$
\sqrt{\nu}=\lambda
$$


so that equation (3.7) becomes

$$
\frac{1}{\sqrt{2 \varepsilon}} \operatorname{tg}\left(\sqrt{\frac{\varepsilon}{2}} \lambda\right)=\operatorname{cotg}(\lambda(1-\varepsilon)) .
$$

The odd eigenfunctions satisfy

$$
\left\{\begin{array}{l}
-u^{\prime \prime}=\nu\left(1+\frac{1}{2 \varepsilon} \chi_{(-\varepsilon, 0)}\right) u \quad \text { in }(-1,0) \\
u(-1)=u(0)=0 .
\end{array}\right.
$$

It is easy to see that the corresponding eigenvalues, under the change of unknown (3.8), satisfy

$$
\sqrt{2 \varepsilon} \operatorname{tg}\left(\sqrt{\frac{\varepsilon}{2}} \lambda\right)=-\operatorname{tg}(\lambda(1-\varepsilon)) .
$$

Let us first analyze the roots $\lambda$ such that the terms on the left hand sides of (3.9) and (3.11) are positive. Thus, we focus first on the interval $0<\lambda<\pi / \sqrt{2 \varepsilon}$. The intervals in which the right hand sides of (3.9) and (3.11) are positive are, respectively,

$$
\begin{gathered}
I_{i}=\left(\frac{i \pi}{1-\varepsilon}, \frac{\left(i+\frac{1}{2}\right) \pi}{1-\varepsilon}\right), i \in \mathbb{Z} \\
J_{i}=\left(\frac{(i-1 / 2) \pi}{1-\varepsilon}, \frac{i \pi}{1-\varepsilon}\right), i \in \mathbb{Z} .
\end{gathered}
$$

Observe that these intervals are disjoint and that $I_{i}$ is in between $J_{i}$ and $J_{i+1}$.

It is easy to see that equations (3.9) and (3.11) have at most one root in the intervals (3.12) and (3.13) respectively. This shows that, in order to prove (3.5) it is sufficient to get lower bounds on the distance between $\lambda_{k}^{\varepsilon}$ and the extremes of the intervals in which they lie.

Let us consider first the root on $I_{i}$. Since the function on the left hand side is increasing and the one on the right hand side decreasing, it is easy to see that, as $\lambda$ increases it approaches the left extreme of the interval $I_{i}$. Given $a>0$, to see that the roots of (3.9) are at distance greater than $\sqrt{a \varepsilon}$ to the left extreme of $I_{i}$ it is sufficient to impose that

$$
\frac{1}{\sqrt{2 \varepsilon}} \operatorname{tg}\left(\sqrt{\frac{\varepsilon}{2}} \lambda\right) \leq \operatorname{cotg}(\sqrt{a \varepsilon}(1-\varepsilon)),
$$

or, equivalently,

$$
\lambda \leq \sqrt{\frac{2}{\varepsilon}} \operatorname{arctg}(\sqrt{2 \varepsilon} \operatorname{cotg}(\sqrt{a \varepsilon}(1-\varepsilon))) .
$$

On the other hand, in order to guarantee that the distance between the roots of (3.11) and the right extreme of the intervals $J_{i}$ in which they lie is greater than $\sqrt{b \varepsilon}$ the following is a sufficient condition:

$$
\sqrt{2 \varepsilon} \operatorname{tg}\left(\sqrt{\frac{\varepsilon}{2}} \lambda\right) \geq-\operatorname{tg}\left(\frac{\pi}{2}-\sqrt{b \varepsilon}(1-\varepsilon)\right)
$$


or, equivalently,

$$
\lambda \geq \sqrt{\frac{2}{\varepsilon}} \operatorname{arctg}\left(\frac{1}{\sqrt{2 \varepsilon}} \operatorname{tg}(\sqrt{b \varepsilon}(1-\varepsilon))\right) .
$$

We now claim that there exist $a, b, c>0$ such that

$$
\sqrt{\frac{2}{\varepsilon}} \operatorname{arctg}(\sqrt{2 \varepsilon} \operatorname{cotg}(\sqrt{a \varepsilon}(1-\varepsilon)))-\sqrt{\frac{2}{\varepsilon}} \operatorname{arctg}\left(\frac{\operatorname{tg}(\sqrt{b \varepsilon}(1-\varepsilon))}{\sqrt{2 \varepsilon}}\right)>c .
$$

Indeed, using Taylor's developments at $\varepsilon=0$ we have:

$$
\frac{1}{\sqrt{2 \varepsilon}} \operatorname{tg}(\sqrt{b \varepsilon}(1-\varepsilon))=\sqrt{\frac{b}{2}}+O(\varepsilon) ; \sqrt{2 \varepsilon} \operatorname{cotg}(\sqrt{a \varepsilon}(1-\varepsilon))=\sqrt{\frac{2}{a}}+O(\varepsilon) \text {. }
$$

By the mean value theorem, there exists $\eta(\varepsilon) \in\left(\sqrt{\frac{2}{a}}+O(\varepsilon), \sqrt{\frac{b}{2}}+O(\varepsilon)\right)$ such that

$$
\begin{aligned}
& \sqrt{\frac{2}{\varepsilon}}\left(\operatorname{arctg}(\sqrt{2 \varepsilon} \operatorname{cotg}(\sqrt{a \varepsilon}(1-\varepsilon)))-\operatorname{arctg}\left(\frac{1}{\sqrt{2 \varepsilon}} \operatorname{tg}(\sqrt{b \varepsilon}(1-\varepsilon))\right)\right. \\
& =\sqrt{\frac{2}{\varepsilon}}\left(\frac{1}{1+\eta^{2}(\varepsilon)}\right)\left(\sqrt{\frac{b}{2}}-\sqrt{\frac{2}{a}}+O(\varepsilon)\right) \\
& \geq \sqrt{\frac{2}{\varepsilon}}\left(\frac{1}{1+b / 2+O(\varepsilon)}\right)\left(\sqrt{\frac{b}{2}}-\sqrt{\frac{2}{a}}+O(\varepsilon)\right) .
\end{aligned}
$$

Clearly, the last term can be done uniformly greater than a positive constant $c>0$ for $\varepsilon>0$ small enough provided $a$ and $b$ are taken such that $\sqrt{b / 2}-$ $\sqrt{2 / a}>0$.

Now, fix $a, b>0$ as above. Then, clearly, two consecutive eigenvalues (solutions of (3.9) and (3.11)) may not be simultaneously to a distance less than $\sqrt{a \varepsilon}$ and $\sqrt{b \varepsilon}$ respectively of the left extreme of $I_{i}$ and the right extreme of $J_{i}$. Indeed, otherwise, in view of the conditions (3.14) and (3.15) we have obtained, the quantity of the left hand side of (3.16) would be bounded above by $(\sqrt{a}+\sqrt{b}) \sqrt{\varepsilon}$ and this is in contradiction with the positivity of the constant $c$ in (3.16).

On the other hand, since the roots of (3.9) move much faster towards the left end of $I_{i}$ than the roots of (3.11) move to the left end of $J_{i}$ as $\lambda$ increases, it is easy to see that the situation we have considered is precisely that in which the gap is minimized.

More precisely, in order to guarantee that the roots of (3.11) are at a distance greater than $\frac{\pi}{4}(1-\varepsilon)$ of the left extreme of $J_{i}$, it is sufficient to guarantee that

$$
\sqrt{2 \varepsilon} \operatorname{tg}\left(\sqrt{\frac{\varepsilon}{2}} \lambda\right) \leq-\operatorname{tg}\left(\frac{\pi}{2}+\frac{\pi}{4}\right)=1
$$

or, equivalently,

$$
\lambda \leq \sqrt{\frac{2}{\varepsilon}} \operatorname{arctg}\left(\frac{1}{\sqrt{2 \varepsilon}}\right) .
$$


For $\varepsilon>0$ small enough, the right hand side of (3.17) is greater than the right hand side of (3.14), i.e.

$$
\sqrt{\frac{2}{\varepsilon}} \operatorname{arctg}[\sqrt{2 \varepsilon} \operatorname{cotg}(\sqrt{a \varepsilon}(1-\varepsilon))] \leq \sqrt{\frac{2}{\varepsilon}} \operatorname{arctg}\left(\frac{1}{\sqrt{2 \varepsilon}}\right) .
$$

In a similar way, it is easy to check that the roots of (3.9) are at a distance greater than $\frac{\pi}{4(1-\varepsilon)}$ of the right extreme of $I_{i}$ if

$$
\lambda \geq \sqrt{\frac{2}{\varepsilon}} \operatorname{arctg}(\sqrt{2 \varepsilon})
$$

and, again, for sufficiently small $\varepsilon>0$,

$$
\sqrt{\frac{2}{\varepsilon}} \operatorname{arctg}\left[\frac{1}{\sqrt{2 \varepsilon}} \operatorname{tg}(\sqrt{b \varepsilon}(1-\varepsilon))\right] \geq \sqrt{\frac{2}{\varepsilon}} \operatorname{arctg}(\sqrt{2 \varepsilon}) .
$$

In view of (3.16)-(3.18) we deduce that

$$
\sqrt{\frac{2}{\varepsilon}}\left[\operatorname{arctg}\left(\frac{1}{\sqrt{2 \varepsilon}}\right)-\operatorname{arctg}(\sqrt{2 \varepsilon})\right] \geq c>0
$$

too and, as above, this implies that when measuring the distance from a root of (3.9) to the next root of (3.11) this is at least of the order of $\frac{\pi}{4}(1-\varepsilon)$. Therefore, the proof of the lower bound (3.5) for the eigenvalues in the interval $(0, \pi / \sqrt{2 \varepsilon})$ is concluded.

The same argument can be used to bound the gap between roots in the intervals of the form $\left(\frac{\sqrt{2} k \pi}{\sqrt{\varepsilon}}, \frac{(2 k+1) \pi}{\sqrt{2 \varepsilon}}\right)$ in which the terms on the right hand side of (3.9) and (3.11) remain positive.

The distance between the roots on the intervals $\left(\frac{(2 k+1) \pi}{\sqrt{2 \varepsilon}}, \frac{\sqrt{2}(k+1) \pi}{\sqrt{\varepsilon}}\right)$ in which the terms on the left hand sides of (3.9) and (3.11) are negative can be estimated in a similar way.

Let us now prove the upper bound on the gap:

$$
\min _{j \neq k}\left|\lambda_{j}-\lambda_{k}\right| \leq C_{2} \sqrt{\varepsilon}
$$

We focus on the eigenvalues in the interval $0<\lambda<\pi / \sqrt{2 \varepsilon}$. We will use the following simple lemma:

LEMMA 3.2. Let $\left\{\alpha_{k}\right\}_{k>1}$ be an increasing sequence of positive real numbers. Assume that there exist positive constants $a, b, A, B$ with $B>A$ such that the following three conditions hold:

$$
\begin{aligned}
& \text { If } A<k \pi /(1-\varepsilon), \text { then } \alpha_{2 k+1}-k \pi /(1-\varepsilon)<a \\
& \text { If } k \pi /(1-\varepsilon)<B \text { then } k \pi /(1-\varepsilon)-\alpha_{2 k}<b \\
& B-A>\pi /(1-\varepsilon) .
\end{aligned}
$$

Then, necessarily

$$
\min _{k \geq 1}\left(\alpha_{k+1}-\alpha_{k}\right)<a+b
$$


Proof. By (3.22), there exists $k \geq 1$ such that $A<k \pi /(1-\varepsilon)<B$ and therefore $\alpha_{2 k+1}, \alpha_{2 k}$ are in the conditions of (3.20) and (3.21) respectively. Thus

$$
\alpha_{2 k+1}-\alpha_{2 k}<\alpha_{2 k+1}-k \pi /(1-\varepsilon)+k \pi /(1-\varepsilon)-\alpha_{2 k}<a+b .
$$

We denote by $\left\{\lambda_{2 k-1}\right\}_{k>1}$ the roots of (3.9) in the intervals $I_{i}$ and by $\left\{\lambda_{2 k}\right\}_{k>1}$ the roots of (3.11) in the intervals $J_{i}$. It is sufficient to check that, given any $a>0, b>0$, then:

If $\frac{k \pi}{1-\varepsilon}>\sqrt{\frac{2}{\varepsilon}} \operatorname{arctg}(\sqrt{2 \varepsilon} \operatorname{cotg}(\sqrt{a \varepsilon}(1-\varepsilon)))$, then $\lambda_{2 k+1}-k \pi(1-\varepsilon)<\sqrt{a \varepsilon}$;

$$
\text { If } \frac{k \pi}{1-\varepsilon}<\sqrt{\frac{2}{\varepsilon}} \operatorname{arctg}\left(\frac{1}{\sqrt{2 \varepsilon}} \operatorname{tg}(\sqrt{b \varepsilon}(1-\varepsilon))\right) \text {, then } \frac{k \pi}{1-\varepsilon}-\lambda_{2 k}<\sqrt{b \varepsilon} .
$$

Indeed, assuming that (3.24) and (3.25) hold and applying Lemma 3.2 it would be sufficient to show the existence of $a, b>0$ such that

$$
\begin{aligned}
& \sqrt{\frac{2}{\varepsilon}} \operatorname{arctg}\left(\frac{1}{\sqrt{2 \varepsilon}} \operatorname{tg}(\sqrt{a \varepsilon}(1-\varepsilon))\right)-\sqrt{\frac{2}{\varepsilon}} \operatorname{arctg}(\sqrt{2 \varepsilon} \operatorname{cotg}(\sqrt{b \varepsilon}(1-\varepsilon))) \\
& >\pi /(1-\varepsilon), \forall 0<\varepsilon<1
\end{aligned}
$$

but this can be done in a straightforward way by using Taylor's expansion at $\varepsilon=0$.

Finally, let us check that (3.24) holds, the proof of (3.25) being analogous. We recall that the roots of (3.9) are at a distance smaller than $\sqrt{a \varepsilon}$ to the left extreme of $I_{i}$ if

$$
\lambda \geq \sqrt{\frac{2}{\varepsilon}} \operatorname{arctg}[\sqrt{2 \varepsilon} \operatorname{cotg}(\sqrt{a \varepsilon}(1-\varepsilon))] .
$$

Then, clearly, since $\lambda_{2 k+1}>k \pi /(1-\varepsilon)$ and $\frac{k \pi}{(1-\varepsilon)}$ is the left extreme of the interval in which $\lambda_{2 k+1}$ lies, if

$$
\frac{k \pi}{1-\varepsilon} \geq \sqrt{\frac{2}{\varepsilon}} \operatorname{arctg}[\sqrt{2 \varepsilon} \operatorname{cotg}(\sqrt{a \varepsilon}(1-\varepsilon))]
$$

then, necessarilly, $\lambda_{2 k+1}-k \pi /(1-\varepsilon)<\sqrt{a \varepsilon}$.

REMARK 3.3. From the proof of Theorem 3.1 it can be seen that the qualitative behavior of the eigenvalues is different in the intervals in which the left hand sides of (3.9) and (3.11) are positive and negative. Indeed, when the gap is minimized the expressions of (3.9) and (3.11) being positive, the roots of (3.9) approach the left extreme of the interval in which they lie while the roots of (3.11) approach the right end of the intervals $J_{i}$. However, in the regions in which the left hand side of (3.9) and (3.11) are negative the reverse happens, i.e. the roots of (3.9) approach the right end while the roots of (3.11) approach the left one. This behavior was refered to as "solotone" phenomena in [8].

Notice that when the density of the string is of the form $\rho(x)=1+$ $\alpha(x)$ with $\alpha$ smooth, non-negative, bounded and such that $\int_{-1}^{1} \alpha=1$ then the eigenvalues are equidistributed at high frequencies. More precisely, Esaim: COCV, July 1997, Vol.2, PP. 231-280 
$\sqrt{\nu_{k}} \sim k \pi /(2 \sqrt{2})$ and the asymptotic gap is of the order of $\pi /(2 \sqrt{2})$ (see [10]). The solotone behavior is due to the lack of regularity of the density in our problem.

\subsection{The limit problem}

The eigenvalue problem associated with (1.1) consists on finding $\nu$ such that

$$
\left\{\begin{array}{l}
-u^{\prime \prime}=\nu u \\
u\left(0^{+}\right)=u\left(0^{-}\right) \\
u^{\prime}\left(0^{-}\right)-u^{\prime}\left(0^{+}\right)=\nu u(0)
\end{array}\right.
$$

admits a non-trivial solution $u$. One can easily check that the eigenvalues constitute an increasing sequence of positive real numbers

$$
0<\nu_{1}<\nu_{2}<\cdots<\nu_{k}<\cdots \rightarrow \infty
$$

and that the corresponding eigenfunctions $\left\{\phi_{k}\right\}_{k \geq 1}$ form an orthonormal basis of $H_{0}^{1}(-1,1)$.

Indeed, $\nu_{k}=\mu_{k}^{-1}, \mu_{k}$ being the eigenvalues of the compact, self-adjoint operator $T: H_{0}^{1}(-1,1) \rightarrow H_{0}^{1}(-1,1)$ such that $T f=u, u \in H_{0}^{1}(-1,1)$ being the unique solution of

$$
\left\{\begin{array}{l}
u \in H_{0}^{1}(-1,1) \\
\int_{-1}^{1} u^{\prime} v^{\prime} d x=\int_{-1}^{1} f v d s+f(0) v(0), \forall v \in H_{0}^{1}(-1,1) .
\end{array}\right.
$$

The symmetry of the problem allows to show that the eigenfunctions are either even or odd. Even eigenfunctions solve

$$
\left\{\begin{array}{l}
u^{\prime \prime}+\nu u=0 \quad \text { in }(0,1) \\
u^{\prime}(0)+\frac{\nu}{2} u(0)=u(1)=0
\end{array}\right.
$$

which, under the change of unknown $\nu=\lambda^{2}$, reduces to the equation $\lambda=$ $2 \operatorname{cotg}(\lambda)$. Let us denote by

$$
0<\omega_{1}<\omega_{2}<\cdots<\omega_{k}<\cdots
$$

the sequence of roots of this equation.

Odd eigenfunctions satisfy

$$
\left\{\begin{array}{l}
u^{\prime \prime}+\nu u=0 \quad \text { in }(0,1) \\
u(0)=u(1)=0
\end{array}\right.
$$

and therefore the corresponding eigenvalues and eigenfunctions are

$$
\begin{cases}\nu_{2 k}=k^{2} \pi^{2}, & k \geq 1 \quad\left(\lambda_{2 k}=\sqrt{\nu_{2 k}}=k \pi\right), \\ \phi_{2 k}(x)=\sin (k \pi x), & k \geq 1 .\end{cases}
$$

It is easy to see that

$$
\sqrt{\nu_{2 k-2}}=\lambda_{2 k-2}<\lambda_{2 k-1}=\sqrt{\nu_{2 k-1}}=\omega_{k}<\lambda_{2 k}=\sqrt{\nu_{2 k}} .
$$

On the other hand, a simple calculation shows that

$$
\lambda_{2 k}-\lambda_{2 k-1}=k \pi-\omega_{k}=\frac{2}{k \pi}+O\left(k^{-3}\right), \text { as } k \rightarrow \infty .
$$

In particular, the spectral gap in the limit system (1.1) vanishes, i.e.

$$
\inf _{j \neq k}\left|\sqrt{\nu_{j}}-\sqrt{\nu_{k}}\right|=0 \text {. }
$$


This is consistent with Theorem 3.1, namely the spectral gap in the approximate system (1.2) vanishes as $\varepsilon \rightarrow 0$.

\section{Convergence of the spectrum}

First of all we obtain some preliminary convergence results by means of basic tools from Functional Analysis. In a second paragraph we prove refined convergence results by a precise asymptotic analysis of the eigenvalue problems.

\subsection{Preliminary Results}

Let us recall that $T_{\varepsilon}, T \in \mathcal{L}\left(H_{0}^{1}(-1,1), H_{0}^{1}(-1,1)\right)$ are the linear, selfadjoint compact operators such that their eigenvalues $\left\{\mu_{k}^{\varepsilon}\right\}_{k \geq 1},\left\{\mu_{k}\right\}_{k \geq 1}$ satisfy $\mu_{k}^{\varepsilon}=1 / \nu_{k}^{\varepsilon}, \mu_{k}=1 / \nu_{k}$, where $\left\{\nu_{k}^{\varepsilon}\right\}_{k \geq 1}$ and $\left\{\nu_{k}\right\}_{k>1}$ are respectively the eigenvalues of (3.1) and (3.26). We have the following result on the convergence of $T_{\varepsilon}$ towards $T$.

Proposition 4.1. There exists $C>0$ such that

$$
\left\|T_{\varepsilon}-T\right\|_{\mathcal{L}\left(H_{0}^{1}(-1,1), H_{0}^{1}(-1,1)\right)} \leq C \sqrt{\varepsilon}, \forall 0<\varepsilon<1 .
$$

Proof. Given $f \in H_{0}^{1}(-1,1)$ we set $T_{\varepsilon} f=u^{\varepsilon}, T f=u$. We have

$$
\begin{aligned}
& \left\|\left(T^{\varepsilon}-T\right) f\right\|_{H_{0}^{1}(-1,1)}^{2}=\int_{-1}^{1}\left|u_{x}^{\varepsilon}-u_{x}\right|^{2} d x=\int_{-1}^{1} u_{x}^{\varepsilon}\left(u_{x}^{\varepsilon}-u_{x}\right) \\
& \quad-\int_{-1}^{1} u_{x}\left(u_{x}^{\varepsilon}-u_{x}\right)=\int_{-1}^{\varepsilon} f\left(u^{\varepsilon}-u\right)+\int_{-\varepsilon}^{\varepsilon} \frac{f\left(u^{\varepsilon}-u\right)}{2 \varepsilon}+\int_{\varepsilon}^{1} f\left(u^{\varepsilon}-u\right) \\
& \quad-\int_{-1}^{1} f\left(u^{\varepsilon}-u\right)-f(0)\left(u^{\varepsilon}-u\right)(0)=-\int_{-\varepsilon}^{\varepsilon} f\left(u^{\varepsilon}-u\right) \\
& \quad+\int_{-\varepsilon}^{\varepsilon} \frac{f\left(u^{\varepsilon}-u\right)-f(0)\left(u^{\varepsilon}-u\right)(0)}{2 \varepsilon} \leq 2 \varepsilon\left\|u^{\varepsilon}-u\right\|_{\infty}\|f\|_{\infty} \\
& +\sqrt{2 \varepsilon}\left(\int_{-\varepsilon}^{\varepsilon} \frac{\left|f\left(u^{\varepsilon}-u\right)-f(0)\left(u^{\varepsilon}-u\right)(0)\right|^{2}}{4 \varepsilon^{2}} d x\right)^{1 / 2}
\end{aligned}
$$

Using Hardy's inequality the last term can be estimated as follows:

$$
\begin{aligned}
& \int_{-\varepsilon}^{\varepsilon}\left|f\left(u^{\varepsilon}-u\right)-f(0)\left(u^{\varepsilon}-u\right)(0)\right|^{2} d x \\
& \quad=\int_{-\varepsilon}^{\varepsilon} \frac{\left|f\left(u^{\varepsilon}-u\right)-f(0)\left(u^{\varepsilon}-u\right)(0)\right||x|^{2}}{|x|^{2}} d x \\
& \quad \leq \varepsilon^{2} \int_{-\varepsilon}^{\varepsilon} \frac{\left|f\left(u^{\varepsilon}-u\right)-f(0)\left(u^{\varepsilon}-u\right)(0)\right|^{2}}{|x|^{2}} d x \\
& \leq C \varepsilon^{2} \int_{-1}^{1}\left|\left(f\left(u^{\varepsilon}-u\right)\right)_{x}\right|^{2} d x
\end{aligned}
$$

Combining (4.2) and (4.4) we deduce that

$$
\begin{aligned}
\left\|\left(T^{\varepsilon}-T\right) f\right\|_{H_{0}^{1}}^{2} & \leq 2 \varepsilon\left\|u^{\varepsilon}-u\right\|_{\infty}\|f\|_{\infty}+C \sqrt{\varepsilon}\left\|f\left(u^{\varepsilon}-u\right)\right\|_{H_{0}^{1}(-1,1)} \\
& \leq C \sqrt{\varepsilon}\|f\|_{H_{0}^{1}(-1,1)}\left\|u^{\varepsilon}-u\right\|_{H_{0}^{1}(-1,1)}
\end{aligned}
$$


This concludes the proof of Proposition 4.1.

As a consequence of this proposition and of classical results on the continuity of the spectrum (see, for instance, E. Sánchez Palencia [11], page 227) we have:

Proposition 4.2. For all $k \geq 1, \nu_{k}^{\varepsilon}$ converges to $\nu_{k}$ as $\varepsilon \rightarrow 0$. On the other hand, the eigenfunctions $\phi_{k}^{\varepsilon}$ converge strongly in $H_{0}^{1}(-1,1)$ to $\phi_{k}$ as $\varepsilon \rightarrow 0$.

Using the variational principle that characterizes the eigenvalues we can obtain the following result on the rate of convergence of eigenvalues.

Proposition 4.3. There exists a constant $C>0$ such that

$$
\left|\frac{1}{\nu_{k}^{\varepsilon}}-\frac{1}{\nu_{k}}\right| \leq C \sqrt{\varepsilon}, \forall k \geq 1, \forall \varepsilon>0 .
$$

REMARK 4.4. Observe that, strictly speaking, (4.5) does not provide a uniform estimate on the rate of convergence of the eigenvalues.

Proof. We proceed as in [3]. We know that

$$
\nu_{k}^{\varepsilon}=\sup _{P \in E_{k}} \inf _{\substack{u \in P \perp \\ u \neq 0}} \frac{\int_{-1}^{1}\left|u^{\prime}\right|^{2}}{\int_{-1}^{-\varepsilon} u^{2}+\frac{1}{2 \varepsilon} \int_{-\varepsilon}^{\varepsilon} u^{2}+\int_{\varepsilon}^{1} u^{2}} ; \nu_{k}=\sup _{P \in E_{k}} \inf _{\substack{u \in P \perp \\ u \neq 0}} \frac{\int_{-1}^{1}\left|u^{\prime}\right|^{2}}{\int_{-1}^{1} u^{2}+u^{2}(0)}
$$

where $E_{k}$ is the set of $(k-1)$-dimensional subspaces of $H_{0}^{1}(-1,1)$ and $P^{\perp}$ its orthogonal complement. Observe that

$$
\begin{aligned}
& \int_{-1}^{-\varepsilon} u^{2}+\frac{1}{2 \varepsilon} \int_{-\varepsilon}^{\varepsilon} u^{2}+\int_{\varepsilon}^{1} u^{2} \\
& \quad \leq \int_{-1}^{1} u^{2}+u^{2}(0)+\left|\left(1-\frac{1}{2 \varepsilon}\right) \int_{-\varepsilon}^{\varepsilon} u^{2}+u^{2}(0)\right| .
\end{aligned}
$$

Proceeding as in the proof of Proposition 4.1 we can show that

$$
\left|\left(1-\frac{1}{2 \varepsilon}\right) \int_{-\varepsilon}^{\varepsilon} u^{2} d x+u^{2}(0)\right| \leq C \sqrt{\varepsilon}\|u\|_{H_{0}^{1}(-1,1)}^{2} .
$$

Therefore

$$
\begin{aligned}
\frac{1}{\nu_{k} \varepsilon} & =\inf _{P \in E_{k}} \sup _{\substack{u \in P \perp \\
u \neq 0}}\left[\frac{\int_{-1}^{-\varepsilon} u^{2}+\frac{1}{2 \varepsilon} \int_{-\varepsilon}^{\varepsilon} u^{2}+\int_{\varepsilon}^{1} u^{2}}{\int_{-1}^{1}\left|u^{\prime}\right|^{2}}\right] \\
& \leq \inf _{P \in E_{k}} \sup _{\substack{u \in P \perp \\
u \neq 0}}\left[\frac{\int_{-1}^{1} u^{2}+u^{2}(0)}{\int_{-1}^{1}\left|u^{\prime}\right|^{2}}+C \sqrt{\varepsilon}\right]=\frac{1}{\nu_{k}}+C \sqrt{\varepsilon}, \forall \varepsilon>0, \forall k \geq 1 .
\end{aligned}
$$

This concludes the proof of (4.5).

As a consequence of these estimates we have the following preliminary result on the rate of convergence of the eigenfunctions:

Proposition 4.5. There exists a positive constant $C>0$ such that for any $K \in \mathbb{N}$

$$
\left\|\phi_{k}^{\varepsilon}-\phi_{k}\right\|_{H_{0}^{1}(-1,1)} \leq C K^{4} \sqrt{\varepsilon}, \quad \forall k \leq K, \quad \forall \varepsilon<K^{-8}
$$


Proof. First of all we observe that

$$
\left\|T \phi_{k}^{\varepsilon}-\frac{1}{\nu_{k}^{\varepsilon}} \phi_{k}^{\varepsilon}\right\|_{H_{0}^{1}(-1,1)}=\left\|T \phi_{k}^{\varepsilon}-T^{\varepsilon} \phi_{k}^{\varepsilon}\right\|_{H_{0}^{1}(-1,1)} \leq C \sqrt{\varepsilon}
$$

by Proposition 4.1. This allows us to apply the following classical result in spectral theory (see, for instance, Oleinik, Shamaev and Yosifian [9]):

Proposition 4.6. Let $T: H \rightarrow H$ be a linear self-adjoint compact operator in a Hilbert space $H$. Assume that there exists $\mu>0$ and $u \in H$ with $\|u\|_{H}=1$ such that

$$
\|T u-\mu u\|_{H} \leq \alpha
$$

with $\alpha>0$. Then, for every $\beta>\alpha$ there exists some $\widetilde{u} \in H$ with $\|\widetilde{u}\|_{H}=1$ such that

$$
\|u-\widetilde{u}\|_{H} \leq 2 \frac{\alpha}{\beta}
$$

$\widetilde{u}$ being a finite linear combination of eigenvectors of $T$ corresponding to eigenvalues in the interval $[\mu-\beta, \mu+\beta]$.

In view of (4.7) we are going to apply Proposition 4.6 with $\alpha=C \sqrt{\varepsilon}$. We need to find $\beta>C \sqrt{\varepsilon}$ such that the unique eigenvalue of $T$ in the interval $\left[1 / \nu_{k}^{\varepsilon}-\beta, 1 / \nu_{k}^{\varepsilon}+\beta\right]$ is $1 / \nu_{k}$. To do this, first of all we estimate the quantity

$$
\begin{aligned}
& m_{K}=\min _{k \leq K}\left\{\left|\frac{1}{\nu_{k+1}}-\frac{1}{\nu_{k}}\right|,\left|\frac{1}{\nu_{k}}-\frac{1}{\nu_{k-1}}\right|\right\} \\
& =\min _{k \leq K}\left\{\frac{\sqrt{\nu_{k+1}}+\sqrt{\nu_{k}}}{\nu_{k+1} \nu_{k}}\left|\sqrt{\nu_{k+1}}-\sqrt{\nu_{k}}\right|, \frac{\sqrt{\nu_{k}}+\sqrt{\nu_{k-1}}}{\nu_{k} \nu_{k-1}}\left|\sqrt{\nu_{k}}-\sqrt{\nu_{k-1}}\right|\right\} .
\end{aligned}
$$

In view of the results of section 3.2 it is easy to see that, for $\delta>0$ sufficiently small:

$$
\min _{k \leq K}\left\{\frac{\sqrt{\nu_{k+1}}+\sqrt{\nu_{k}}}{\nu_{k+1} \nu_{k}}, \frac{\sqrt{\nu_{k}}+\sqrt{\nu_{k-1}}}{\nu_{k} \nu_{k-1}}\right\} \geq \delta K^{-3} .
$$

Therefore, since $\sqrt{\nu_{k}}-\sqrt{\nu_{k-1}}$ is of the order of $k^{-1}$ as $k \rightarrow \infty$ we deduce that, for a sufficiently small $\delta>0, m_{K} \geq \delta K^{-4}$. Taking $\beta$ such that $C \sqrt{\varepsilon}<$ $\beta<\delta K^{-4}$, in view of Proposition 4.6, we deduce that

$$
\left\|\phi_{k}-\phi_{k}^{\varepsilon}\right\|_{H_{0}^{1}(-1,1)} \leq \frac{2 C \sqrt{\varepsilon}}{\beta}
$$

and minimizing the upper bound we conclude that (4.5) holds for a suitable choice of $C>0$.

\subsection{Refined estimates on the Rate of COnVERgence}

Concerning the rate of convergence of the eigenvalues of (1.2) towards those of the limit system we have the following:

Theorem 4.7. We have

$$
\begin{aligned}
& \left|\sqrt{\nu_{2 k}^{\varepsilon}}-k \pi\right|<3 \sqrt{\varepsilon}+O(\varepsilon), \forall k<(1-\varepsilon) / \pi \sqrt{2 \varepsilon}=O\left(\varepsilon^{-1 / 2}\right) \\
& \left|\sqrt{\nu_{2 k-1}^{\varepsilon}}-\omega_{k}\right|<2 \sqrt{2 \varepsilon}+O\left(\varepsilon^{5 / 6}\right), \forall k<\varepsilon^{-1 / 6}
\end{aligned}
$$


Proof. First we consider the eigenvalues $\nu_{2 k}^{\varepsilon}$ that correspond to odd eigenfunctions. From section 3 we know that

$$
\sqrt{\nu_{2 k}^{\varepsilon}} \in\left[\frac{(k-1 / 2) \pi}{1-\varepsilon}, \frac{k \pi}{1-\varepsilon}\right] .
$$

On the other hand, if $0<x<1 / \sqrt{2 \varepsilon}$ we have $0<\sqrt{2 \varepsilon} \operatorname{tg}\left(\sqrt{\frac{\varepsilon}{2}} x\right)<\sqrt{2 \varepsilon}$ and therefore, taking $k<(1-\varepsilon) / \pi \sqrt{2 \varepsilon}$ we obtain $0<\sqrt{2 \varepsilon} \operatorname{tg}\left(\sqrt{\frac{\varepsilon}{2}} \sqrt{\nu_{2 k}^{\varepsilon}}\right)<\sqrt{2 \varepsilon}$. Taking into account that $\sqrt{\nu_{2 k}^{\varepsilon}}$ solves (3.10) we deduce that

$$
0<-\operatorname{tg}\left(\sqrt{\nu_{2 k}^{\varepsilon}}(1-\varepsilon)\right)<\sqrt{2 \varepsilon}
$$

and therefore, using the Taylor expansion of $\operatorname{arctg}(x)$ at $x=0$,

$$
-k \pi<-\sqrt{\nu_{2 k}^{\varepsilon}}(1-\varepsilon)<\operatorname{arctg}(\sqrt{2 \varepsilon})=-k \pi+\sqrt{2 \varepsilon}+O(\varepsilon) .
$$

Therefore

$$
\left|\sqrt{\nu_{2 k}^{\varepsilon}}-\frac{k \pi}{1-\varepsilon}\right| \leq \sqrt{2 \varepsilon}+O(\varepsilon)
$$

and then, if $k<(1-\varepsilon) / \pi \sqrt{2 \varepsilon}$ we have

$$
\begin{aligned}
&\left|\sqrt{\nu_{2 k}^{\varepsilon}}-k \pi\right| \leq \sqrt{2 \varepsilon}+O(\varepsilon)+\left|\frac{k \pi}{1-\varepsilon}-k \pi\right|=\sqrt{2 \varepsilon}+\frac{k \varepsilon \pi}{1-\varepsilon}+O(\varepsilon) \\
&<\left(\sqrt{2}+\frac{1}{\sqrt{2}}\right) \sqrt{\varepsilon}+O(\varepsilon)<3 \sqrt{\varepsilon}+O(\varepsilon) .
\end{aligned}
$$

Let us consider now the eigenvalues $\nu_{2 k-1}^{\varepsilon}$ corresponding to even eigenfunctions. From section 3 we know that

$$
\sqrt{\nu_{2 k-1}^{\varepsilon}} \in\left[\frac{(k-1) \pi}{1-\varepsilon}, \frac{(k-1 / 2) \pi}{1-\varepsilon}\right] \text {. }
$$

Using the Taylor expansion of $\operatorname{tg}(x)$ at $x=0$ we get that

$$
\frac{\sqrt{\nu_{2 k-1}^{\varepsilon}}}{2}<\frac{1}{\sqrt{2 \varepsilon}} \operatorname{tg}\left(\sqrt{\frac{\varepsilon}{2}} \sqrt{\nu_{2 k-1}^{\varepsilon}}\right)<\frac{\sqrt{\nu_{2 k-1}^{\varepsilon}}}{2}+\sqrt{\varepsilon}+O\left(\varepsilon^{7 / 6}\right)
$$

provided $\left(\nu_{2 k-1}^{\varepsilon}\right)^{3 / 2} / 6<1 / \sqrt{\varepsilon}$ and therefore, in particular, if $k<\frac{1}{2}+$ $\frac{(1-\varepsilon)}{\pi}\left(\frac{6}{\sqrt{\varepsilon}}\right)^{1 / 3}$. Taking into account that $\sqrt{\nu_{2 k-1}^{\varepsilon}}$ are the roots of $(3.7)$ we deduce that

$$
0<\operatorname{cotg}\left(\sqrt{\nu_{2 k-1}^{\varepsilon}}(1-\varepsilon)\right)-\frac{\sqrt{\nu_{2 k-1}^{\varepsilon}}}{2}<\sqrt{\varepsilon}+O\left(\varepsilon^{7 / 6}\right)
$$

If $\omega_{k}^{\varepsilon}$ is the $k$-th positive root of $\operatorname{cotg}(x(1-\varepsilon))=\frac{x}{2}$, using the Taylor expansion of the function $f_{\varepsilon}(x)=\operatorname{cotg}((1-\varepsilon) x)-\frac{x}{2}$ at $\omega_{k}^{\varepsilon}$, in view of (4.10) we deduce that

$$
\left|\sqrt{\nu_{2 k-1}^{\varepsilon}}-\omega_{k}^{\varepsilon}\right|<2 \sqrt{\varepsilon}+O(\varepsilon) .
$$

On the other hand, as seen in section $3.2, \omega_{k}$ is the root of $\operatorname{cotg}(x)=\frac{x}{2}$. It is easy to check that

$$
\left|\omega_{k}^{\varepsilon}-\omega_{k}\right|<C \varepsilon k
$$


Therefore

$$
\left|\sqrt{\nu_{2 k-1}^{\varepsilon}}-\omega_{k}\right| \leq\left|\sqrt{\nu_{2 k-1}^{\varepsilon}}-\omega_{k}^{\varepsilon}\right|+\left|\omega_{k}^{\varepsilon}-\omega_{k}\right|<2 \sqrt{\varepsilon}+C \varepsilon k+O(\varepsilon) .
$$

REMARK 4.8. This result, although it applies to small frequencies only, improves the previous ones in this range. Indeed from Proposition 4.3 we may deduce that

$$
\begin{aligned}
C \sqrt{\varepsilon} & \geq\left|\frac{1}{\nu_{k}^{\varepsilon}}-\frac{1}{\nu_{k}}\right|=\left|\frac{1}{\sqrt{\nu_{k}^{\varepsilon}}}-\frac{1}{\sqrt{\nu_{k}}}\right|\left|\frac{1}{\sqrt{\nu_{k}^{\varepsilon}}}+\frac{1}{\sqrt{\nu_{k}}}\right| \\
& \geq\left|\frac{1}{\sqrt{\nu_{k}^{\varepsilon}}}-\frac{1}{\sqrt{\nu_{k}}}\right|\left(\frac{2(1-\varepsilon)}{k \pi}+\frac{2}{k \pi}\right) \geq C k^{-1}\left|\frac{1}{\sqrt{\nu_{k}^{\varepsilon}}}-\frac{1}{\sqrt{\nu_{k}}}\right| .
\end{aligned}
$$

Therefore

$$
\left|\sqrt{\nu_{k}^{\varepsilon}}-\sqrt{\nu_{k}}\right|=\sqrt{\nu_{k}^{\varepsilon}} \sqrt{\nu_{k}}\left|\frac{1}{\sqrt{\nu_{k}^{\varepsilon}}}-\frac{1}{\sqrt{\nu_{k}}}\right| \leq C k^{3} \sqrt{\varepsilon} .
$$

Clearly, the results of Theorem 4.7 improve significantly (4.11). The fact that estimates of the form (4.8) and (4.9) hold for small frequencies only is natural in view of the solotone phenomena described in Remark 3.3.

We have a similar result for the eigenfunctions:

TheOREM 4.9. There exist positive constants $C>0, \delta>0$ such that

$$
\left\|\phi_{2 k}^{\varepsilon}-\phi_{2 k}\right\|_{H_{0}^{1}(-1,1)}<C \sqrt{\varepsilon}, \forall k<\delta \varepsilon^{-1 / 2}, \forall 0<\varepsilon<1
$$

and

$$
\left\|\phi_{2 k-1}^{\varepsilon}-\phi_{2 k-1}\right\|_{H_{0}^{1}(-1,1)}<C \sqrt{\varepsilon}, \forall k<\delta \varepsilon^{-1 / 6}, \forall 0<\varepsilon<1 .
$$

Proof. A straightforward computation shows that the eigenfunctions of the approximate problem normalized in $H_{0}^{1}(-1,1)$ (in the norm $\left(\int_{-1}^{1}\left|u^{\prime}\right|^{2}\right.$ $d x)^{1 / 2}$ ) are as follows

$$
\begin{gathered}
\phi_{2 k}^{\varepsilon}=\left\{\begin{array}{l}
-\frac{\rho_{2 k}^{\varepsilon}}{\lambda_{2 k}^{\varepsilon}} \sin \left(\lambda_{2 k}^{\varepsilon}(1+x)\right), x \in(-1,-\varepsilon) \\
\frac{\rho_{2 k}^{\varepsilon}}{\lambda_{2 k}^{\varepsilon}} \gamma_{2 k}^{\varepsilon} \sin \left(\frac{\lambda_{2 k}^{\varepsilon}}{\sqrt{2 \varepsilon}} x\right), x \in(-\varepsilon, \varepsilon) \\
\frac{\rho_{2 k}^{\varepsilon}}{\lambda_{2 k}^{\varepsilon}} \sin \left(\lambda_{2 k}^{\varepsilon}(1-x)\right), x \in(\varepsilon, 1)
\end{array}\right. \\
\phi_{2 k-1}^{\varepsilon}=\left\{\begin{array}{l}
\frac{\rho_{2 k-1}^{\varepsilon}}{\lambda_{2 k-1}^{\varepsilon}} \sin \left(\lambda_{2 k-1}^{\varepsilon}(1+x)\right), x \in(-1,-\varepsilon) \\
\frac{\rho_{2 k-1}^{\varepsilon}}{\lambda_{2 k-1}^{2}} \gamma_{2 k-1}^{\varepsilon} \cos \left(\frac{\lambda_{2 k-1}^{\varepsilon}}{\sqrt{2 \varepsilon}} x\right), x \in(-\varepsilon, \varepsilon) \\
\frac{\rho_{2 k-1}^{\varepsilon}}{\lambda_{2 k-1}^{\varepsilon}} \sin \left(\lambda_{2 k-1}^{\varepsilon}(1-x)\right), x \in(\varepsilon, 1)
\end{array}\right.
\end{gathered}
$$


with

$$
\begin{aligned}
\rho_{k}^{\varepsilon} & =\left[1-\varepsilon+\frac{\sin \left(2 \lambda_{k}^{\varepsilon}(1-\varepsilon)\right)}{2 \lambda_{k}^{\varepsilon}}+\left(\frac{1}{2}+(-1)^{k} \frac{\sin \left(\sqrt{2 \varepsilon} \lambda_{k}^{\varepsilon}\right)}{2 \sqrt{2 \varepsilon} \lambda_{k}^{\varepsilon}}\right)\left(\gamma_{k}^{\varepsilon}\right)^{2}\right]^{-1 / 2} \\
\gamma_{2 k}^{\varepsilon} & =-\sqrt{2 \varepsilon} \frac{\cos \left(\lambda_{2 k}^{\varepsilon}(1-\varepsilon)\right)}{\cos \left(\lambda_{2 k}^{\varepsilon} \sqrt{\frac{\varepsilon}{2}}\right)} ; \quad \gamma_{2 k-1}^{\varepsilon}=\frac{\sin \left(\lambda_{2 k-1}^{\varepsilon}(1-\varepsilon)\right)}{\cos \left(\lambda_{2 k-1}^{\varepsilon} \sqrt{\frac{\varepsilon}{2}}\right)} .
\end{aligned}
$$

Recall that $\lambda_{k}^{\varepsilon}=\sqrt{\nu_{k}^{\varepsilon}}$. In these expressions we have chosen the derivative of the eigenfuction at $x=1$ to be negative.

On the other hand, the normalized eigenfunctions of the limit problem are given by

$$
\phi_{2 k}=\frac{(-1)^{k+1}}{k \pi} \sin (k \pi x) ; \quad \phi_{2 k-1}= \begin{cases}\frac{\rho_{k}}{\omega_{k}} & \sin \left(\omega_{k}(1+x)\right), x \in(-1,0) \\ \frac{\rho_{k}}{\omega_{k}} & \sin \left(\omega_{k}(1-x)\right), x \in(0,1)\end{cases}
$$

with $\rho_{k}=\left(1+\frac{\sin \left(2 \omega_{k}\right)}{2 \omega_{k}}\right)^{-1 / 2}$. This eigenfunction has been chosen to satisfy the same sign criteria at $x=1$.

Using the fact that $\phi_{2 k}^{\varepsilon}$ and $\phi_{2 k}$ are odd functions we have

$$
\begin{aligned}
\| & \phi_{2 k}^{\varepsilon}-\phi_{2 k}||_{H_{0}^{1}(-1,1)}^{2}=\int_{-1}^{1}\left|\left(\phi_{2 k}^{\varepsilon}\right)^{\prime}-\phi_{2 k}^{\prime}\right|^{2} d x=2 \int_{0}^{1}\left|\left(\phi_{2 k}^{\varepsilon}\right)^{\prime}-\phi_{2 k}^{\prime}\right|^{2} d x \\
= & 2 \int_{0}^{\varepsilon}\left|\frac{\rho_{2 k}^{\varepsilon} \gamma_{2 k}^{\varepsilon}}{\sqrt{2 \varepsilon}} \cos \left(\frac{\lambda_{2 k}^{\varepsilon}}{\sqrt{2 \varepsilon}} x\right)-(-1)^{k+1} \cos (k \pi x)\right|^{2} \\
& +2 \int_{\varepsilon}^{1}\left|\rho_{2 k}^{\varepsilon} \cos \left(\lambda_{2 k}^{\varepsilon}(1-x)\right)+(-1)^{k+1} \cos (k \pi x)\right|^{2} \\
\leq & 4 \int_{0}^{\varepsilon}\left[\left|\frac{\rho_{2 k}^{\varepsilon} \gamma_{2 k}^{\varepsilon}}{\sqrt{2 \varepsilon}} \cos \left(\frac{\lambda_{2 k}^{\varepsilon} x}{\sqrt{2 \varepsilon}}\right)\right|^{2}+|\cos (k \pi x)|^{2}\right] \\
& +4 \int_{\varepsilon}^{1}\left[\left|\rho_{2 k}^{\varepsilon}-1\right|^{2}\left|\cos \left(\lambda_{2 k}^{\varepsilon}(1-x)\right)\right|^{2}\right] \\
& +4 \int_{\varepsilon}^{1}\left[\left|\cos \left(\lambda_{2 k}^{\varepsilon}(1-x)\right)-\cos (k \pi(1-x))\right|^{2}\right] \\
& \leq 2\left|\rho_{2 k}^{\varepsilon} \gamma_{2 k}^{\varepsilon}\right|^{2}+4 \varepsilon+4\left|\rho_{2 k}^{\varepsilon}-1\right|^{2}(1-\varepsilon) \\
& +4\left|\lambda_{2 k}^{\varepsilon}-k \pi\right|^{2} \int_{\varepsilon}^{1}(1-x)^{2} \sin ^{2}(\xi(x)) d x \leq 2\left|\rho_{2 k}^{\varepsilon} \gamma_{2 k}^{\varepsilon}\right|^{2}+4 \varepsilon \\
& +4\left|\rho_{2 k}^{\varepsilon}-1\right|^{2}(1-\varepsilon)+4\left|\lambda_{2 k}^{\varepsilon}-k \pi\right|^{2} \frac{(1-\varepsilon)^{3}}{3}
\end{aligned}
$$

Let us analyze now all the terms on the right hand side of this inequality. First we observe that if $k<1 / \pi \sqrt{2 \varepsilon}$, then

$$
\cos \left(\lambda_{2 k}^{\varepsilon} \sqrt{\frac{\varepsilon}{2}}\right)>\cos \left(k \pi \sqrt{\frac{\varepsilon}{2}}\right)>\cos \left(\frac{1}{2}\right)>\frac{1}{2}
$$

and therefore

$$
\left|\gamma_{2 k}^{\varepsilon}\right|^{2}=2 \varepsilon \frac{\cos ^{2}\left(\lambda_{2 k}^{\varepsilon}(1-\varepsilon)\right)}{\cos ^{2}\left(\lambda_{2 k}^{\varepsilon} \sqrt{\frac{\varepsilon}{2}}\right)}<8 \varepsilon
$$


On the other hand, it is easy to check that $\left(\rho_{2 k}^{\varepsilon}\right)^{2}<\frac{C}{1-\varepsilon}$ when $k<1 / \pi \sqrt{2 \varepsilon}$. The last term in (4.14) can be estimated as a consequence of Theorem 4.7.

Let us analyze finally the term

$$
\begin{aligned}
& \left|\rho_{2 k}^{\varepsilon}-1\right|^{2}=\mid \frac{1}{\sqrt{1-\varepsilon}}\left(1+\frac{\sin \left(2 \lambda_{2 k}^{\varepsilon}(1-\varepsilon)\right)}{2 \lambda_{2 k}^{\varepsilon}(1-\varepsilon)}\right) \\
& \quad+\left(1+\frac{\sin \left(\sqrt{2 \varepsilon} \lambda_{2 k}^{\varepsilon}\right)}{\sqrt{2 \varepsilon} \lambda_{2 k}^{\varepsilon}} \frac{\left(\gamma_{2 k}^{\varepsilon}\right)^{2}}{2(1-\varepsilon)}\right)^{-1 / 2}-\left.1\right|^{2} \\
& \quad \leq 2\left|\frac{1}{\sqrt{1-\varepsilon}}-1\right|^{2}+\frac{2}{1-\varepsilon} \mid\left(1+\frac{\sin \left(2 \lambda_{2 k}^{\varepsilon}(1-\varepsilon)\right)}{2 \lambda_{2 k}^{\varepsilon}(1-\varepsilon)}+\right. \\
& \left.\quad+\left(1+\frac{\sin \left(\sqrt{2 \varepsilon} \lambda_{2 k}^{\varepsilon}\right)}{\sqrt{2 \varepsilon} \lambda_{2 k}^{\varepsilon}}\right) \frac{\left(\gamma_{2 k}^{\varepsilon}\right)^{2}}{2(1-\varepsilon)}\right)^{-1 / 2}-\left.1\right|^{2} .
\end{aligned}
$$

The first term on the right hand side is clearly of the order of $\varepsilon^{2}$. The second one can be bounded above by $C \varepsilon$ as follows. First of all we observe that

$$
\left|1-\frac{1}{\sqrt{1+x}}\right| \leq \frac{|x|}{2} \text { if } x>0 .
$$

On the other hand

$$
\begin{aligned}
\left|\frac{\sin \left(2 \lambda_{2 k}^{\varepsilon}(1-\varepsilon)\right)}{2 \lambda_{2 k}^{\varepsilon}(1-\varepsilon)}\right| & =\left|\frac{\sin \left(2 \lambda_{2 k}^{\varepsilon}(1-\varepsilon)-2 k \pi\right)}{2 \lambda_{2 k}^{\varepsilon}(1-\varepsilon)}\right| \leq\left|\frac{\lambda_{2 k}^{\varepsilon}(1-\varepsilon)-k \pi}{\lambda_{2 k}^{\varepsilon}(1-\varepsilon)}\right| \\
& =\left|\frac{\lambda_{2 k}^{\varepsilon}-k \pi}{\lambda_{2 k}^{\varepsilon}(1-\varepsilon)}-\frac{\varepsilon}{\lambda_{2 k}^{\varepsilon}(1-\varepsilon)}\right|=O(\sqrt{\varepsilon})
\end{aligned}
$$

and

$$
\begin{gathered}
\left|\left(\frac{1}{2}+\frac{\sin \left(\sqrt{2 \varepsilon} \lambda_{2 k}^{\varepsilon}\right)}{2 \sqrt{2 \varepsilon} \lambda_{2 k}^{\varepsilon}}\right) \frac{\left(\gamma_{2 k} \varepsilon\right)^{2}}{1-\varepsilon}\right|^{\leq} \leq \frac{\left(\gamma_{2 k} \varepsilon\right)^{2}}{1-\varepsilon} \\
=\frac{2 \varepsilon}{1-\varepsilon}\left(\frac{\cos \left(\lambda_{2 k}^{\varepsilon}(1-\varepsilon)\right)}{\cos \left(\lambda_{2 k}^{\varepsilon} \sqrt{\frac{\varepsilon}{2}}\right)}\right)^{2}=O(\varepsilon)
\end{gathered}
$$

in view of (4.8). Combining (4.15)-(4.18) we easily deduce that $\left|\rho_{2 k}^{\varepsilon}-1\right|^{2}=$ $O(\varepsilon)$.

The proof of (4.13) is rather similar and we omit it for brevity.

\section{WELL-POSEDNESS OF THE LIMIT PROBLEM IN THE ASYMMETRIC SPACE}

As we have shown in section 3.2 the eigenvalues $\nu_{k}$ are divided in two sets: $\left\{k^{2} \pi^{2}\right\}_{k \in \mathbb{N}}$ and $\left\{\omega_{k}^{2}\right\}_{k \in \mathbb{N}}$ corresponding to odd and even eigenfunctions respectively. In this section we change a bit the notation in what concerns the eigenfunctions: $\phi_{k}$ is an eigenfunction associated to the eigenvalue $k^{2} \pi^{2}$ while $\psi_{k}$ is associated to $\omega_{k}^{2}$. The set $\left\{\phi_{k}\right\}_{k \in \mathbb{N}} \cup\left\{\psi_{k}\right\}_{k \in \mathbb{N}}$ is chosen so that they constitute an orthonormal basis of $H_{0}^{1}(-1,1)$.

Esaim: COCV, JUly 1997, Vol.2, PP. 231-280 
The solution $u$ of (1.1) can be written as follows

$$
\begin{aligned}
u(x, t)=\sum_{k \in \mathbb{N}}\{ & {\left[\widetilde{a}_{k} \cos (k \pi t)+\widetilde{c}_{k} \sin (k \pi t)\right] \phi_{k}(x) } \\
& \left.+\left[\widetilde{b}_{k} \cos \left(\omega_{k} t\right)+\widetilde{d}_{k} \sin \left(\omega_{k} t\right)\right] \psi_{k}(x)\right\}
\end{aligned}
$$

where $\widetilde{a}_{k}, \widetilde{b}_{k}, \widetilde{c}_{k}, \widetilde{d}_{k}$ are real Fourier coefficients determined by the initial data:

$$
\left\{\begin{array}{l}
u(x, 0)=u_{0}(x)=\sum_{k \in \mathbb{N}}\left(\widetilde{a}_{k} \phi_{k}+\widetilde{b}_{k} \psi_{k}\right) \\
u_{t}(x, 0)=u_{1}(x)=\sum_{k \in \mathbb{N}}\left(k \pi \widetilde{c}_{k} \phi_{k}+\omega_{k} \pi \widetilde{d}_{k} \psi_{k}\right) \\
u_{t}(0,0)=z_{1}=\sum_{k \in \mathbb{N}}\left(k \pi \widetilde{c}_{k} \phi_{k}(0)+\omega_{k} \pi \widetilde{d}_{k} \psi_{k}(0)\right) .
\end{array}\right.
$$

This section is organized as follows. In a first subsection we will give some basic properties of the elliptic operator involved in system (1.1). This will allow us to explain how the coefficients $\widetilde{a}_{k}, \widetilde{b}_{k}, \widetilde{c}_{k}, \widetilde{d}_{k}$ of the Fourier development can be computed since, in principle, system (5.2) seems to be overdetermined. In a second subsection we will introduce an asymmetric Fourier space in which the system (1.1) is well-posed. Finally, we will characterize this space in classical terms and see that it is constituted (roughly) by functions with one more degree of regularity to one side of the mass.

\subsection{Preliminaries on the development of solutions in FOURIER SERIES}

Let us introduce the domains of the fractional powers of the compact self-adjoint operator $T \in \mathcal{L}\left(H_{0}^{1}(-1,1), H_{0}^{1}(-1,1)\right)$ that we have introduced in section 3.2 to show the existence of the eigenvalues of the limit system. For any $\alpha \in \mathbb{R}$ we define the Hilbert space.

$$
\begin{aligned}
X_{\alpha}=\{u & =\sum_{k \in \mathbb{N}}\left(a_{k} \phi_{k}+b_{k} \psi_{k}\right): \\
& \left.\|u\|_{\alpha}^{2}=\sum_{k \in \mathbb{N}}\left\{\left|a_{k}\right|^{2} k^{4 \alpha}+\left|b_{k}\right|^{2} \omega_{k}^{4 \alpha}\right\}<\infty\right\},
\end{aligned}
$$

endowed with the norm $\|\cdot\|_{\alpha}$. Taking into account that $\left\{\phi_{k}\right\} \cup\left\{\psi_{k}\right\}$ have been chosen to constitute an orthonormal basis of $H_{0}^{1}(-1,1)$ we deduce that

$$
X_{0}=H_{0}^{1}(-1,1) \text {. }
$$

On the other hand, clearly

$$
\begin{aligned}
& X_{1}=\operatorname{Im}\left(T^{-1}\right)=\left\{u \in\left[H^{3}(-1,0) \times H^{3}(0,1)\right] \cap H_{0}^{1}(-1,1):\right. \\
& \left.u^{\prime \prime}( \pm 1)=0, u^{\prime \prime}\left(0^{+}\right)=u^{\prime \prime}\left(0^{-}\right)=u^{\prime}\left(0^{+}\right)-u^{\prime}\left(0^{-}\right)\right\} \text {, }
\end{aligned}
$$

the norm in $X_{1}$ being equivalent to

$$
\|u\|_{1}=\left\|T^{-1} u\right\|_{H_{0}^{1}(-1,1)}=\left[\int_{-1}^{0}\left|u^{\prime \prime \prime}\right|^{2}+\int_{0}^{1}\left|u^{\prime \prime \prime}\right|^{2}\right]^{1 / 2} .
$$


Let us characterize now $X_{1 / 2}$. Given $u \in X_{1}$ we have

$$
\begin{aligned}
\|u\|_{1 / 2}^{2} & =\left\langle T^{-1} u, u>_{H_{0}^{1}(-1,1)}=-\int_{-1}^{0} u^{\prime \prime \prime} u^{\prime}-\int_{0}^{1} u^{\prime \prime \prime} u^{\prime}\right. \\
& =\int_{-1}^{1}\left|u^{\prime \prime}\right|^{2}+\left|u^{\prime}\left(0^{+}\right)-u^{\prime}\left(0^{-}\right)\right|^{2} .
\end{aligned}
$$

Therefore $X_{1 / 2}=\left[H^{2}(-1,0) \times H^{2}(0,1)\right] \cap H_{0}^{1}(-1,1)$. In a similar way we may compute $X_{-1 / 2}$. We have

$$
\|u\|_{-1 / 2}^{2}=<T u, u>_{H_{0}^{1}(-1,1)}=\int_{-1}^{1} u^{2}+u^{2}(0) .
$$

If we identify $H_{0}^{1}(-1,1)$ with a closed subspace of $H_{0}^{1}(-1,1) \times \mathbb{R}$ by means of the linear mapping $u \rightarrow(u, u(0))$, we see that the norms of the spaces $X_{-1 / 2}$ and $L^{2}(-1,1) \times \mathbb{R}$ coincide over $H_{0}^{1}(-1,1)$. Since $H_{0}^{1}(-1,1)$ is dense in both spaces we deduce that $\left(X_{-1 / 2},\|\cdot\|_{-1 / 2}\right)$ coincides with $L^{2}(-1,1) \times \mathbb{R}$ algebraically and topologically.

With these characterizations of the fractional spaces $X_{\alpha}$ it is easy to see how the coefficients of the Fourier expansion (5.1) are determined in terms of the initial data.

We assume that $\left(u_{0}, u_{1}, z_{1}\right) \in H_{0}^{1}(-1,1) \times L^{2}(-1,1) \times \mathbb{R}$ which coincides with $X_{0} \times X_{-1 / 2}$. Then, since $u_{0} \in H_{0}^{1}(-1,1) \equiv X_{0}$ and $\left\{\phi_{k}\right\} \cup\left\{\psi_{k}\right\}$ constitutes an orthonormal basis of $H_{0}^{1}(-1,1)$ we have

$$
\begin{aligned}
\widetilde{a}_{k} & =<u_{0}, \phi_{k}>_{H_{0}^{1}(-1,1)}=\int_{-1}^{1}\left(u_{0}\right)^{\prime} \phi_{k}^{\prime} d x ; \quad \widetilde{b}_{k}=<u_{0}, \psi_{k}>_{H_{0}^{1}(-1,1)} \\
& =\int_{-1}^{1}\left(u_{0}\right)^{\prime} \psi_{k}^{\prime} d x .
\end{aligned}
$$

On the other hand, since $\left(u_{1}, z_{1}\right) \in L^{2}(-1,1) \times \mathbb{R} \equiv X_{-1 / 2}$ we have

$$
\begin{aligned}
& \widetilde{c}_{k}=<\left(u_{1}, z_{1}\right), \phi_{k}>_{X_{-1 / 2}}=\int_{-1}^{1} u_{1} \phi_{k} d x+z_{1} \phi_{k}(0)=\int_{-1}^{1} u_{1} \phi_{k} d x \\
& \widetilde{d}_{k}=<\left(u_{1}, z_{1}\right), \psi_{k}>_{X_{-1 / 2}}=\int_{-1}^{1} u_{1} \psi_{k} d x+z_{1} \psi_{k}(0) .
\end{aligned}
$$

Under these assumptions it is easy to see that the solution $u$ given by (5.1) belongs to the class $C\left([0, \infty) ; H_{0}^{1}(-1,1)\right) \cap C^{1}\left([0, \infty) ; L^{2}(-1,1)\right)$ and moreover, that

$$
u(0, t)=z(t)=\sum_{k \in \mathbb{N}}\left[\widetilde{b}_{k} \cos \left(\omega_{k} t\right)+\widetilde{d}_{k} \sin \left(\omega_{k} t\right)\right] \psi_{k}(0)
$$

belongs to $C^{1}([0, \infty))$.

In order to simplify the expression for the solution $u$ we set

$$
\begin{aligned}
& \phi_{-k}=\phi_{k}, \quad \psi_{-k}=\psi_{k}, \quad \omega_{-k}=-\omega_{k} \\
& a_{k}=\frac{\widetilde{a}_{k}-i \widetilde{c}_{k}}{2}, \quad a_{-k}=\frac{\widetilde{a}_{k}+i \widetilde{c}_{k}}{2}, \quad b_{k}=\frac{\widetilde{b}_{k}-i \tilde{d}_{k}}{2}, \quad b_{-k}=\frac{\widetilde{b}_{k}+i \tilde{d}_{k}}{2} .
\end{aligned}
$$


Then, clearly

$$
\begin{aligned}
u(x, t) & =\sum_{k \in \mathbb{Z} \backslash\{0\}}\left(a_{k} e^{i k \pi t} \phi_{k}+b_{k} e^{i \omega_{k} t} \psi_{k}\right) \\
z(t)=u(0, t) & =\sum_{k \in \mathbb{Z} \backslash\{0\}} b_{k} e^{i \omega_{k} t} \psi_{k}(0) .
\end{aligned}
$$

On the other hand, $\left(u_{0}, u_{1}, z_{1}\right) \in H_{0}^{1}(-1,1) \times L^{2}(-1,1) \times \mathbb{R}$ if and only if $\left\{a_{k}\right\},\left\{b_{k}\right\} \in \ell^{2}$ or, equivalently,

$u \in C\left([0, \infty) ; H_{0}^{1}(-1,1)\right) \cap C^{1}\left([0, \infty) ; L^{2}(-1,1)\right) ; \quad u(0, t) \in C^{1}([0, \infty))$.

The energy of the solution (which is constant in time) can also be represented in terms of the Fourier coefficients:

$$
\begin{aligned}
E(t) & =\frac{1}{2}\left[\int_{-1}^{1}\left|u_{x}(x, t)\right|^{2}+\int_{-1}^{1}\left|u_{t}(x, t)\right|^{2}+\left|z_{t}(t)\right|^{2}\right] \\
& =\frac{1}{2}\left[\|u(t)\|_{H_{0}^{1}(-1,1)}^{2}+\left\|\left(u_{t}(t), z_{t}(t)\right)\right\|_{L^{2}(-1,1) \times \mathbb{R}}^{2}\right] \\
& =\frac{1}{2} \sum_{k \in \mathbb{Z} \backslash\{0\}}\left(\left|a_{k} e^{i k \pi t}\right|^{2}+\left|b_{k} e^{i \omega_{k} t}\right|^{2}\right) \\
& =\frac{1}{2} \sum_{k \in \mathbb{Z} \backslash\{0\}}\left(\left|a_{k}\right|^{2}+\left|b_{k}\right|^{2}\right)=E(0) .
\end{aligned}
$$

\subsection{The asymmetric Fourier space}

We set

$$
\delta_{k}=\omega_{k+\sigma_{k}}-k \pi
$$

where $\sigma_{k}$ is the sign function, i.e. $\sigma_{k}=1$ if $k>0$ and $\sigma_{k}=-1$ if $k<0$.

In view of the results of section 3.2 we know that

$$
\delta_{k}=O\left(k^{-1}\right) \text {. }
$$

We introduce the Hilbert space

$$
\begin{aligned}
H & =\left\{U=\sum_{k \in \mathbb{Z} \backslash\{0\}}\left(a_{k} \bar{\phi}_{k}+b_{k} \bar{\psi}_{k}\right) \in Y=X_{0} \times X_{-1 / 2}:\|U\|_{H}^{2}=\right. \\
& \left.=\sum_{k \in \mathbb{Z} \backslash\{0\}}\left|a_{k}\right|^{2}+\left|b_{1}\right|^{2}+\left|b_{-1}\right|^{2}+\sum_{k \in \mathbb{Z} \backslash\{0, \pm 1\}} \frac{\left|a_{k-\sigma_{k}}+b_{k}\right|^{2}}{\delta_{k-\sigma_{k}}^{2}}<\infty\right\} .
\end{aligned}
$$

In (5.9) we have used the notation

$$
\bar{\phi}_{k}=\left(\phi_{k}, k \pi \phi_{k}, 0\right) ; \quad \bar{\psi}_{k}=\left(\psi_{k}, \omega_{k} \psi_{k}, \omega_{k} \psi_{k}(0)\right)
$$

so that the vector $U$ represents the vector-valued unknown of our system

$$
U=\left(u, u_{t}, z_{t}\right) \text {. }
$$

Since $\delta_{k} \rightarrow 0$ as $k \rightarrow \infty$ it is clear that

$$
H \subset Y=X_{0} \times X_{-1 / 2}
$$

i.e. $H$ is a strict subspace of the energy space $Y=H_{0}^{1}(-1,1) \times L^{2}(-1,1) \times \mathbb{R}$. Moreover, $H$ is clearly an asymmetric Fourier space since the coefficients 
$\left\{a_{k-\sigma_{k}}-b_{k}\right\}$ and $\left\{a_{k-\sigma_{k}}+b_{k}\right\}$ are weighted differently. Therefore, $H$ is not of the form $X_{\alpha} \times X_{\alpha-1 / 2}$, for any $\alpha \in \mathbb{R}$, i.e. it is not a natural fractional power or energy space for system (1.1). However, system (1.1) is well-posed in this asymmetric Hilbert space:

TheOREM 5.1. For any $\left(u_{0}, u_{1}, z_{1}\right) \in H$ there exists an unique solution $U(t)=\left(u(t), u_{t}(t), z_{t}(t)\right) \in C([0, \infty) ; H)$ of the limit system (1.1). Moreover, there exists $C>0$ such that

$$
\|U(t)\|_{H}^{2} \leq C\left(1+t^{2}\right)\left\|\left(u^{0}, u^{1}, z^{1}\right)\right\|_{H}^{2}, \forall t>0 .
$$

Proof. Given $\left(u_{0}, u_{1}, z_{1}\right) \in H$ the unique solution $U=\left(u, u_{t}, z_{t}\right)$ of (1.1) can be represented in Fourier series

$$
U(t)=\sum_{k \in \mathbb{Z} \backslash\{0\}}\left(a_{k} e^{i k \pi t} \bar{\phi}_{k}+b_{k} e^{i \omega_{k} t} \bar{\psi}_{k}\right)
$$

provided the Fourier coefficients $\left\{a_{k}\right\},\left\{b_{k}\right\}$ are determined by the initial data, i.e.

$$
U_{0}=\left(u_{0}, u_{1}, z_{1}\right)=\sum_{k \in \mathbb{Z} \backslash\{0\}}\left(a_{k} \bar{\phi}_{k}+b_{k} \bar{\psi}_{k}\right) .
$$

On the other hand,

$$
\begin{aligned}
\| U(t) & \|_{H}^{2}=\left|b_{1} e^{i \omega_{1} t}\right|^{2}+\left|b_{-1} e^{i \pi t}\right|^{2}+\sum_{k \in \mathbb{Z} \backslash\{0\}}\left|a_{k} e^{i k \pi t}\right|^{2} \\
& +\sum_{k \in \mathbb{Z} \backslash\{0, \pm 1\}} \frac{\left|a_{k-\sigma_{k}} e^{i\left(k-\sigma_{k}\right) \pi t}+b_{k} e^{i \omega_{k} t}\right|^{2}}{\delta_{k-\sigma_{k}}^{2}} \\
= & \left|b_{1}\right|^{2}+\left|b_{-1}\right|^{2}+\sum_{k \in \mathbb{Z} \backslash\{0\}}\left|a_{k}\right|^{2} \\
& +2 \sum_{k \in \mathbb{Z} \backslash\{0, \pm 1\}}\left(\frac{\left|a_{k-\sigma_{k}}+b_{k}\right|^{2}}{\delta_{k-\sigma_{k}}^{2}}+\left|b_{k}\right|^{2} \frac{\left|e^{i\left(k-\sigma_{k}\right) \pi t}-e^{i \omega_{k} t}\right|^{2}}{\delta_{k-\sigma_{k}}^{2}}\right) \\
\leq & 2\left\|U_{0}\right\|_{H}^{2}+2 \sum_{k \in \mathbb{Z} \backslash\{0, \pm 1\}}\left|b_{k}\right|^{2} \frac{\left|e^{i\left(k-\sigma_{k}\right) \pi t}-e^{i \omega_{k} t}\right|^{2}}{\delta_{k-\sigma_{k}}^{2}} \\
= & 2\left\|U_{0}\right\|_{H}^{2}+2 t^{2} \sum_{k \in \mathbb{Z} \backslash\{0, \pm 1\}}\left|b_{k}\right|^{2} \leq C\left(1+t^{2}\right)\left\|U_{0}\right\|_{H}^{2} .
\end{aligned}
$$

The continuity of $U$ from $t \in[0, \infty)$ to $H$ it is easy to check. This concludes the proof of the theorem.

REMARK 5.2. The proof of this theorem shows that the flow generated by the limit system (1.1) is also stable in every asymmetric space of the form

$$
\begin{aligned}
H_{\alpha}= & \left\{U=\sum_{k \in \mathbb{Z} \backslash\{0\}}\left(a_{k} \bar{\phi}_{k}+b_{k} \bar{\psi}_{k}\right):\left|b_{1}\right|^{2}+\left|b_{-1}\right|^{2}+\sum_{k \in \mathbb{Z} \backslash\{0\}}\left|a_{k}\right|^{2}\right. \\
& \left.+\sum_{k \in \mathbb{Z} \backslash\{0, \pm 1\}} \frac{\left|a_{k-\sigma_{k}}+b_{k}\right|^{2}}{\delta_{k-\sigma_{k}}^{2 \alpha}}<\infty\right\}
\end{aligned}
$$


with $0<\alpha \leq 1$. The space $H$ introduced in (5.9) corresponds to $\alpha=1$ and it is the smallest one in which this holds. It is also obvious that $H$ is the sharp space, in the sense that system (1.1) is not well-posed in $H_{\alpha}$ for any $\alpha>1$.

System (1.1) is also well-posed in a more general class of asymmetric spaces of the form:

$$
\begin{aligned}
& \left\{U=\sum_{k \in \mathbb{Z} \backslash\{0\}}\left(a_{k} \bar{\phi}_{k}+b_{k} \bar{\psi}_{k}\right):\left|b_{1}\right|^{2}+\left|b_{-1}\right|^{2}+\sum_{k \in \mathbb{Z} \backslash\{0\}} \frac{\left|a_{k}\right|^{2}}{\delta_{k}^{2 \alpha}}\right. \\
& \left.+\sum_{k \in \mathbb{Z} \backslash\{0, \pm 1\}} \frac{\left|a_{k-\sigma_{k}}+b_{k}\right|^{2}}{\delta_{k-\sigma_{k}}^{2 \alpha+2}}<\infty\right\}
\end{aligned}
$$

with $\alpha \in \mathbb{R}$. The case we have considered corresponds to $\alpha=0$.

In order to characterize the asymmetric space $H$ it is natural to introduce

$$
p_{k}=\frac{\bar{\psi}_{k}-\bar{\phi}_{k-\sigma_{k}}}{2}, \quad q_{k}=\delta_{k-\sigma_{k}} \frac{\left(\bar{\psi}_{k}+\bar{\phi}_{k-\sigma_{k}}\right)}{2}, \quad \forall k \in \mathbb{Z} \backslash\{0, \pm 1\} .
$$

We have the following result:

Proposition 5.3. The set $\left\{p_{k}\right\}_{k \in \mathbb{Z} \backslash\{0, \pm 1\}} \cup\left\{q_{k}\right\}_{k \in \mathbb{Z} \backslash\{0, \pm 1\}} \cup\left\{\bar{\psi}_{-1}, \bar{\psi}_{1}\right\}$ constitutes a Riesz basis of the asymmetric space $H$.

Proof. We first observe that

$$
\begin{aligned}
& \sum_{k \in \mathbb{Z} \backslash\{0\}}\left(a_{k} \bar{\phi}_{k}+b_{k} \bar{\psi}_{k}\right)=b_{-1} \bar{\psi}_{-1}+b_{1} \bar{\psi}_{1} \\
& +\sum_{k \in \mathbb{Z} \backslash\{0, \pm 1\}}\left(-\left(a_{k-\sigma_{k}}-b_{k}\right) p_{k}+\left(\frac{a_{k-\sigma_{k}}+b_{k}}{\delta_{k-\sigma_{k}}}\right) q_{k}\right) .
\end{aligned}
$$

Therefore, the set under consideration is complete in $H$.

On the other hand, if we define on $H$ a scalar product such that this set is orthonormal, then, clearly, the corresponding norm $\|\cdot\|_{*}$ is such that

$$
\|U\|_{*}^{2}=\left|b_{-1}\right|^{2}+\left|b_{1}\right|^{2}+\sum_{k \in \mathbb{Z} \backslash\{0, \pm 1\}}\left(\left|a_{k-\sigma_{k}}-b_{k}\right|^{2}+\left|\frac{a_{k-\sigma_{k}}+b_{k}}{\delta_{k-\sigma_{k}}}\right|^{2}\right)
$$

which is equivalent to the norm of $H$. This concludes the proof of the proposition.

\subsection{Characterization of the asymmetric space}

The main result of this section is as follows:

TheOREM 5.4. The space $H$ coincides with the subspace of $H_{0}^{1}(-1,1) \times$ $L^{2}(-1,1) \times \mathbb{R}$ constituted by the elements $\left(u_{0}, u_{1}, z_{1}\right)$ such that the restrictions of $\left(u_{0}, u_{1}\right)$ to the interval $(0,1)$ belong to $H^{2}(0,1) \times H^{1}(0,1)$, i.e.

$$
\left(\left.u_{0}\right|_{(0,1)},\left.u_{1}\right|_{(0,1)}\right) \in H^{2}(0,1) \times H^{1}(0,1)
$$

and satisfying the further compatibility conditions

$$
u_{1}\left(0^{+}\right)=z_{1}, u_{1}(1)=0 .
$$


Moreover the norm of $H$ is equivalent to the following one

$$
\left[\left\|u_{0}\right\|_{H_{0}^{1}(-1,1)}^{2}+\left\|u_{1}\right\|_{L^{2}(-1,1)}^{2}+\int_{0}^{1}\left(\left|u_{0, x x}\right|^{2}+\left|u_{1, x}\right|^{2}\right) d x\right]^{1 / 2} .
$$

As a direct consequence of Theorems 5.1 and 5.4 we have

Corollary 5.5. Given $\left(u_{0}, u_{1}, z_{1}\right) \in H_{0}^{1}(-1,1) \times L^{2}(-1,1) \times \mathbb{R}$ such that (5.15)-(5.16) hold, the solution of (1.1), (1.6) satisfies the following further regularity conditions:

$$
\left.u(x, t)\right|_{(0,1)} \in C\left([0, \infty) ; H^{2}(0,1)\right) \cap C^{1}\left([0, \infty) ; H^{1}(0,1)\right)
$$

REMARK 5.6. The regularity result of Corollary 5.1 was proved by S. Hansen and E. Zuazua in [4]. Therefore, the result is not new. However in [4] the proof was based on the use of the explicit formula of solutions of the onedimensional wave equation while the present proof is based on the Fourier expansion method.

Let us now proceed to the proof of Theorem 5.4. First of all we need the following technical lemma:

LEMMA 5.7. For any $k \in \mathbb{Z} \backslash\{0 \pm 1\}$ we set

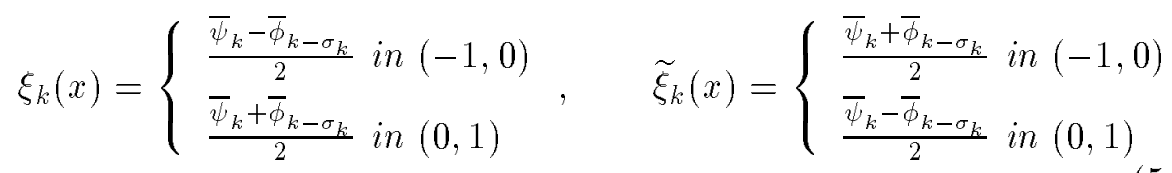

and $\xi_{ \pm 1}=\bar{\psi}_{ \pm 1}$. Then, there exist positive constant $\beta, \gamma>0$ such that the following hold:

$$
\begin{gathered}
\sum_{k \in \mathbb{Z} \backslash\{0\}}\left\|\xi_{k}-\bar{\psi}_{k}\right\|_{X_{0} \times X_{-1 / 2}}^{2}<\infty \\
\sum_{k \in \mathbb{Z} \backslash\{0\}} \frac{1}{k^{2}}\left\|\beta \xi_{k}-\bar{\psi}_{k}\right\|_{X_{1 / 2} \times X_{0}}^{2}<\infty \\
\sum_{k \in \mathbb{Z} \backslash\{0, \pm 1\}}\left\|\widetilde{\xi}_{k}-\frac{\gamma}{k} \bar{\psi}_{k}\right\|_{X_{1 / 2} \times X_{0}}^{2}<\infty
\end{gathered}
$$

where $X_{\alpha}$ are the fractional spaces introduced in section 5.1.

Proof. Let us check that (5.20) holds, the proof of (5.21)-(5.22) being analogous. Note however that in (5.21)-(5.22) the constants $\beta, \gamma$ have to be chosen such that the general terms of the series tend to zero as $k \rightarrow \infty$. 
Functions $\xi_{k}$ and $\bar{\psi}_{k}$ are even. Therefore,

$$
\begin{aligned}
\| \xi_{k} & -\bar{\psi}_{k} \|_{X_{0} \times X_{-1 / 2}}^{2}=\frac{1}{2} \int_{-1}^{0}\left|(-1)^{k} \cos ((k-1) \pi x)+\rho_{k} \cos \left(\omega_{k}(1+x)\right)\right|^{2} \\
& +\frac{1}{2} \int_{-1}^{0}\left|(-1)^{k} \sin ((k-1) \pi x)+\rho_{k} \sin \left(\omega_{k}(1+x)\right)\right|^{2}+\frac{1}{4}\left|\rho_{k} \sin \left(\omega_{k}\right)\right|^{2} \\
\leq & \left(1-\rho_{k}\right)^{2} \int_{-1}^{0}|\cos ((k-1) \pi(1+x) x)|^{2} \\
& +\rho_{k}^{2} \int_{-1}^{0}\left|\cos ((k-1) \pi(1+x))-\cos \left(\omega_{k}(1+x)\right)\right|^{2} \\
& +\left(1-\rho_{k}\right)^{2} \int_{-1}^{0}|\sin ((k-1) \pi(1+x))|^{2} \\
& +\rho_{k}^{2} \int_{-1}^{0}\left|\sin ((k-1) \pi(1+x))-\sin \left(\omega_{k}(1+x)\right)\right|^{2} \\
& +\frac{1}{4}\left|\rho_{k}\right|^{2}\left|\sin \left(\omega_{k}\right)\right|^{2} \leq 2\left(1-\rho_{k}\right)^{2}+\frac{1}{4}\left(\rho_{k} \sin \left(\omega_{k}\right)\right)^{2} \\
& +\rho_{k}^{2} \int_{0}^{1}\left[\left|\cos ((k-1) \pi x)-\cos \left(\omega_{k} x\right)\right|^{2}+\left|\sin ((k-1) \pi x)-\sin \left(\omega_{k} x\right)\right|^{2}\right] .
\end{aligned}
$$

It is easy to see that all the terms on the right hand side of (5.23) are of the order of $k^{-2}$. More precisely, there exists $C>0$ such that $\| \xi_{k}-$ $\bar{\psi}_{k} \|_{X_{0} \times X_{-1 / 2}}^{2} \leq C / k^{2}$ and this implies (5.20).

In view of Proposition 5.3, any element $U \in H$ can be written as

$$
U=d_{1} \bar{\psi}_{1}+d_{-1} \bar{\psi}_{-1}+\sum_{k \in \mathbb{Z} \backslash\{0, \pm 1\}}\left(c_{k} p_{k}+d_{k} q_{k}\right)
$$

with $\left\{c_{k}\right\},\left\{d_{k}\right\} \in \ell^{2}$.

We set $U^{1}=d_{1} \bar{\psi}_{1}+d_{-1} \bar{\psi}_{-1}+\sum_{k \in \mathbb{Z} \backslash\{0, \pm 1\}} d_{k} q_{k}$ and define $U_{e}^{1}$ as the even extension to $(-1,1)$ of the restriction of $U^{1}$ to $(0,1)$. Clearly

$$
U_{e}^{1}=\sum_{k \in \mathbb{Z} \backslash\{0\}} d_{k} \delta_{k-1} \xi_{k}
$$

since $\delta_{k-\sigma_{k}} \xi_{k}$ is the even extension to $(-1,1)$ of the restriction of $q_{k}$ to $(0,1)$.

On the other hand, since $\left\{\bar{\psi}_{k}\right\} \cup\left\{\bar{\phi}_{k}\right\}$ constitute an orthonormal basis of $X_{0} \times X_{-1 / 2}$, then $\left\{\frac{1}{\omega_{k}} \bar{\psi}_{k}\right\} \cup\left\{\frac{1}{k \pi} \bar{\phi}_{k}\right\}$ constitute an orthonormal basis of $X_{1 / 2} \times X_{0}$. 
By (5.21), taking into account that $\bar{\psi}_{k} / \omega_{k}$ are orthonormal in $X_{1 / 2} \times X_{0}$ and that $\omega_{k}^{-1}$ is of the order of $\delta_{k-1}$, we deduce that

$$
\begin{aligned}
& \left\|U_{e}^{1}\right\|_{X_{1 / 2} \times X_{0}}=\left\|\sum_{k \in \mathbb{Z} \backslash\{0\}} d_{k} \delta_{k-\sigma_{k}} \xi_{k}\right\|_{X_{1 / 2} \times X_{0}} \\
& \leq \sum_{k \in \mathbb{Z} \backslash\{0\}} \frac{d_{k} \delta_{k-\sigma_{k}} k\left(\frac{1}{k}\left\|\beta \xi_{k}-\overline{\psi_{k}}\right\|_{X_{1 / 2} \times X_{0}}\right)}{\beta}+\left\|\sum_{k \in \mathbb{Z} \backslash\{0\}} \frac{d_{k} \delta_{k-\sigma_{k}}}{\beta} \bar{\psi}_{k}\right\|_{X_{1 / 2} \times X_{0}} \\
& \leq\left(\sum_{k \in \mathbb{Z} \backslash\{0\}} \frac{\left|d_{k} \delta_{k-\sigma_{k}} k\right|^{2}}{\beta^{2}}\right)^{1 / 2}\left(\sum_{k \in \mathbb{Z} \backslash\{0, \pm 1\}} \frac{1}{k^{2}}\left\|\beta \xi_{k}-\bar{\psi}_{k}\right\|_{X_{1 / 2} \times X_{0}}^{2}\right)^{1 / 2} \\
& \left.\quad+\left\|\sum_{k \in \mathbb{Z} \backslash\{0\}} \frac{d_{k} \delta_{k-\sigma_{k}}}{\beta} \bar{\psi}_{k}\right\|_{X_{1 / 2} \times X_{0}} \sum_{k \in \mathbb{Z} \backslash\{0\}}\left|d_{k}\right|^{2}\right)^{1 / 2} .
\end{aligned}
$$

Therefore $U_{e}^{1} \in X_{1 / 2} \times X_{0}$ and this implies that $\left.U^{1}\right|_{(0,1)} \in H^{2}(0,1) \times H^{1}(0,1)$.

Let us take now $U^{2}=\sum_{k \in \mathbb{Z} \backslash\{0, \pm 1\}} c_{k} p_{k}$ and define $U_{e}^{2}$ as the even extension to $(-1,1)$ of its restriction to $(0,1)$. We have $U_{e}^{2}=\sum_{k \in \mathbb{Z} \backslash\{0, \pm 1\}} c_{k} \widetilde{\xi}_{k}$ and, as above, using (5.23) we obtain

$$
\begin{aligned}
& \left\|U_{e}^{2}\right\|_{X_{1 / 2} \times X_{0}}=\left\|\sum_{k \in \mathbb{Z} \backslash\{0, \pm 1\}} c_{k}\left(\widetilde{\xi}_{k}-\frac{\gamma}{k} \bar{\psi}_{k}\right)\right\|_{X_{1 / 2} \times X_{0}} \\
& +\left\|\sum_{k \in \mathbb{Z} \backslash\{0, \pm 1\}} \frac{\gamma c_{k}}{k} \bar{\psi}_{k}\right\|_{X_{1 / 2} \times X_{0}} \leq C\left(\sum_{k \in \mathbb{Z} \backslash\{0, \pm 1\}}\left|c_{k}\right|^{2}\right)^{1 / 2} .
\end{aligned}
$$

This implies that $\left.U^{2}\right|_{(0,1)} \in H^{2}(0,1) \times H^{1}(0,1)$.

We have proved that, if $U \in H$ then $\left.U\right|_{(0,1)} \in H^{2}(0,1) \times H^{1}(0,1)$. This implies, in particular, that the restriction of $u_{1}$ to $(0,1)$ belongs to $H^{1}(0,1)$ and therefore $u_{1} \in C([0,1])$. As a consequence of this, the compatibility conditions (5.16) hold.

Let us see that every element $U \in X_{0} \times X_{-1 / 2}$ such that (5.15)-(5.16) are satisfied is contained in $H$.

Since $U \in X_{0} \times X_{-1 / 2}$ it can be written as

$$
U=d_{1} \bar{\psi}_{1}+d_{-1} \bar{\psi}_{-1}+\sum_{k \in \mathbb{Z} \backslash\{0, \pm 1\}}\left(c_{k} p_{k}+\frac{d_{k}}{\delta_{k-\sigma_{k}}} q_{k}\right),
$$

with $\left\{c_{k}\right\},\left\{d_{k}\right\} \in \ell^{2}$. In order to see that $U \in H$ we have to show that

$$
\sum_{k \in \mathbb{Z} \backslash\{0, \pm 1\}}\left|\frac{d_{k}}{\delta_{k-\sigma_{k}}}\right|^{2}<\infty
$$

Proceeding as above we can prove that, since $\left\{c_{k}\right\} \in \ell^{2}$, then $U^{2}=d_{1} \bar{\psi}_{1}+$ $d_{-1} \bar{\psi}_{-1}+\sum_{k \in \mathbb{Z} \backslash\{0\}} c_{k} p_{k}$ restricted to $(0,1)$ belongs to $H^{2}(0,1) \times H^{1}(0,1)$ Esaim: CoCV, JUly 1997, Vol.2, PP. 231-280 
and satisfies the compatibility conditions (5.16). Therefore, the problem is reduced to consider elements $U \in X_{0} \times X_{-1 / 2}$ of the form

$$
U=\sum_{k \in \mathbb{Z} \backslash\{0, \pm 1\}} \frac{d_{k}}{\delta_{k-\sigma_{k}}} q_{k}
$$

with $\left\{d_{k}\right\} \in \ell^{2}$ such that (5.15)-(5.16) are satisfied.

Let us define $U_{e}$ the even extension to $(-1,1)$ of the restriction of $U$ to $(0,1)$. In view of the characterizations of the fractional spaces $X_{\alpha}$ of section 5.1 we have that

$$
U_{e}=\sum_{k \in \mathbb{Z} \backslash\{0\}} d_{k} \xi_{k} \in X_{1 / 2} \times X_{0} .
$$

Let us see now that combining (5.21) and (5.25) the proof of (5.24) can be completed.

It is sufficient to show that $\frac{1}{\beta} \sum_{k \in \mathbb{Z} \backslash\{0\}} \omega_{k} d_{k}\left(\frac{\bar{\psi}_{k}}{\omega_{k}}\right)$ belongs to $X_{1 / 2} \times X_{0}$ since $\left\{\bar{\psi}_{k} / \omega_{k}\right\}$ are orthonormal in $X_{1 / 2} \times X_{0}$ and $\omega_{k}^{-1}$ is of the order of $\delta_{k}-\sigma_{k}$.

Actually, it is sufficient to prove the convergence of the tails $\frac{1}{\beta} \sum_{|k|>N} \omega_{k} d_{k}\left(\frac{\bar{\psi}_{k}}{\omega_{k}}\right)$ in $X_{1 / 2} \times X_{0}$ for $N$ large enough. We have

$$
\left\|\sum_{k>|N|} \frac{\omega_{k} d_{k}}{\beta} \frac{\bar{\psi}_{k}}{\omega_{k}}\right\|_{X_{1 / 2} \times X_{0}}=\left(\sum_{|k|>N}\left|\frac{\omega_{k} d_{k}}{\beta}\right|^{2}\right)^{1 / 2} .
$$

On the other hand,

$$
\begin{aligned}
& \left\|\sum_{k>|N|} \frac{\omega_{k} d_{k}}{\beta} \frac{\bar{\psi}_{k}}{\omega_{k}}\right\|_{X_{1 / 2} \times X_{0}} \leq\left\|\sum_{k \in \mathbb{Z} \backslash\{0\}} \frac{k d_{k}}{\beta}\left[\frac{1}{k}\left(\bar{\psi}_{k}-\beta \xi_{k}\right)\right]\right\|_{X_{1 / 2} \times X_{0}} \\
& \quad+\left\|\sum_{k \in \mathbb{Z} \backslash\{0\}} d_{k} \xi_{k}\right\|_{X_{1 / 2} \times X_{0}} \\
& \leq\left(\sum_{|k|>N}\left|\frac{k d_{k}}{\beta}\right|^{2}\right)^{1 / 2}\left(\sum_{|k|>N} \frac{1}{k^{2}}\left\|\beta \xi_{k}-\bar{\psi}_{k}\right\|_{X_{1 / 2} \times X_{0}}^{2}\right)^{1 / 2} \\
& \quad+\left\|\sum_{|k|>N} d_{k} \xi_{k}\right\|_{X_{1 / 2} \times X_{0}}
\end{aligned}
$$

We have

$$
\left\|\sum_{|k|>N} d_{k} \xi_{k}\right\|_{X_{1 / 2} \times X_{0}}<\infty
$$

and, in view of (5.21),

$$
\sum_{|k|>N} \frac{1}{k^{2}}\left\|\beta \xi_{k}-\bar{\psi}_{k}\right\|_{X_{1 / 2} \times X_{0}}^{2} \rightarrow 0, \text { as } N \rightarrow \infty .
$$


Combining (5.26)-(5.28) with $N$ large enough such that

$$
\sum_{|k|>N} \frac{1}{k^{2}}\left\|\beta \xi_{k}-\bar{\psi}_{k}\right\|_{X_{1 / 2} \times X_{0}}^{2}<\frac{\delta}{2}
$$

with $\delta>0$ sufficiently small such that $\left|\delta^{1 / 2} k\right|<\left|\omega_{k}\right|$ for every $|k|>N$, we easily obtain an upper bound for the quantity in (5.24).

This completes the proof of the fact that $H$ coincides algebraically with the space of elements of $X_{0} \times X_{-1 / 2}$ satisfying (5.15)-(5.16). On the other hand, the proof of the first inclusion provides a bound of the norm of the latter in terms of the norm in $H$. The fact that these spaces coincide topologically is then a consequence of the open mapping theorem.

REMARK 5.8. The fact that $H$ coincides with the space of finite energy data with one more degree of regularity to the right of the point mass can be explained, roughly, in the following way: The graphs of $p_{k}=\left(\bar{\psi}_{k}-\bar{\phi}_{k-\sigma_{k}}\right) / 2$ and $q_{k} / \delta_{k-\sigma_{k}}=\left(\bar{\psi}_{k}+\bar{\phi}_{k-\sigma_{k}}\right) / 2$ are basically one reflexion of the other with respect to $x=0$. Moreover $\left.p_{k}\right|_{(0,1)}$ is very small in $H^{1}(0,1) \times L^{2}(0,1)$ while $\left.p_{k}\right|_{(-1,0)}$ is rather close to an orthonormal basis of $H^{1}(-1,0) \times L^{2}(-1,0)$. In a similar way $\left.\frac{q_{k}}{\delta_{k-1}}\right|_{(-1,0)}$ is rather small in $H^{1}(-1,0) \times L^{2}(-1,0)$ while $\left.\frac{q_{k}}{\delta_{k}-\sigma_{k}}\right|_{(0,1)}$ is almost an orthonormal basis of $H^{1}(0,1) \times L^{2}(0,1)$. Since $\delta_{k}=O\left(k^{-1}\right)$ and we are considering $q_{k}$ instead of $q_{k} / \delta_{k-\sigma_{k}}$, we see that $\left.q_{k}\right|_{(-1,0)}$ has to be small in $H^{2}(-1,0) \times H^{1}(-1,0)$ while $\left.q_{k}\right|_{(0,1)}$ is close to an orthonormal basis of $H^{2}(0,1) \times H^{1}(0,1)$. This explains the asymmetry of $H$.

In figures 1 and 2 below we show the graphs of the first components of $\bar{\phi}_{k}$ and $\bar{\psi}_{k-\sigma_{k}}$. Then, in figures 3 and 4 the graphs of the first components of $p_{k}$ and $q_{k} / \delta_{k-\sigma_{k}}$ are shown. These figures exhibit the phenomena we have described above.

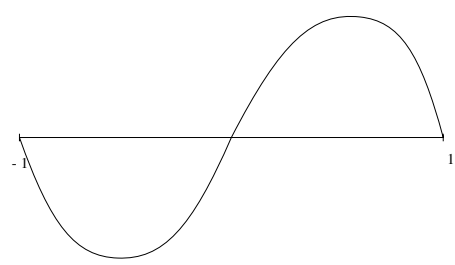

Figure $1: \bar{\phi}_{1}$

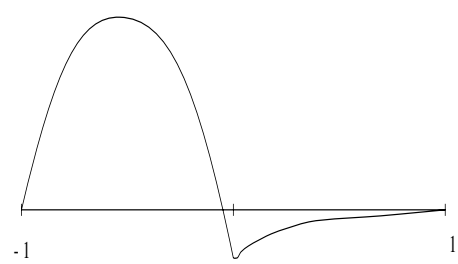

Figure $3: \bar{p}_{2}$

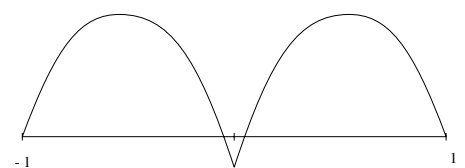

Figure $2: \bar{\psi}_{2}$

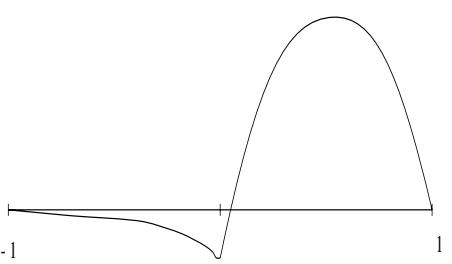

Figure $4: \bar{q}_{2} / \delta_{1}$ 


\section{UNIFORM WELL-POSEDNESS OF THE APPROXIMATE PROBLEMS IN "ASYMMETRIC SPACES"}

The goal of this section is to show that the well-posedness of the limit system in the asymmetric space $H$ can be obtained as a consequence of an uniform well-posedness result of the approximate problems in finite dimensional spaces that, as $\varepsilon \rightarrow 0$, cover the whole asymmetric space $H$. Obviously, the approximate problems (1.2) are not well-posed in an asymmetric space like $H$. Therefore, it is natural to consider them in suitable finite-dimensional spaces.

With a notation similar to that of section 5, solutions $u^{\varepsilon}$ of (1.2) can be written in Fourier series as follows:

$$
u^{\varepsilon}(x, t)=\sum_{k \in \mathbb{Z} \backslash\{0\}}\left(a_{k} e^{i \alpha_{k}^{\varepsilon} t} \phi_{k}^{\varepsilon}(x)+b_{k} e^{i \beta_{k}^{\varepsilon} t} \psi_{k}^{\varepsilon}(x)\right) .
$$

In $(6.1),\left\{\phi_{k}^{\varepsilon}\right\}$ denote the odd eigenfunctions and $\alpha_{k}^{\varepsilon}$ the corresponding eigenvalues while $\left\{\psi_{k}^{\varepsilon}\right\}$ denote the even eigenfunctions and $\beta_{k}^{\varepsilon}$ its eigenvalues. These eigenfunctions are assumed to be orthonormal in $H_{0}^{1}(-1,1)$. The complex Fourier coefficients $\left\{a_{k}\right\} \cup\left\{b_{k}\right\}$ are determined by the initial data and finite energy solutions correspond to $\ell^{2}$ coefficients.

We also introduce the vector-valued eigenfunctions

$$
\bar{\phi}_{k}^{\varepsilon}=\left(\phi_{k}^{\varepsilon}, i \alpha_{k}^{\varepsilon} \phi_{k}^{\varepsilon}\right), \bar{\psi}_{k}^{\varepsilon}=\left(\psi_{k}^{\varepsilon}, i \beta_{k}^{\varepsilon} \psi_{k}^{\varepsilon}\right)
$$

so that the vector valued unknown $U^{\varepsilon}=\left(u^{\varepsilon}, u_{t}^{\varepsilon}\right)$ can be written as follows

$$
U^{\varepsilon}(x, t)=\left(u^{\varepsilon}(x, t), u_{t}^{\varepsilon}(x, t)\right)=\sum_{k \in \mathbb{Z} \backslash\{0\}}\left(a_{k} e^{i \alpha_{k}^{\varepsilon} t} \bar{\phi}_{k}^{\varepsilon}+b_{k} e^{i \beta_{k}^{\varepsilon} t} \bar{\psi}_{k}^{\varepsilon}\right) .
$$

The second components of $\left\{\bar{\phi}_{k}^{\varepsilon}\right\} \cup\left\{\bar{\psi}_{k}^{\varepsilon}\right\}$ constitute an orthonormal basis of $L^{2}(-1,1)$ with the scalar product associated to $(1.2)$, i.e.

$$
|f|_{\varepsilon}=\left(\int_{-1}^{-\varepsilon}|f|^{2} d x+\frac{1}{2 \varepsilon} \int_{-\varepsilon}^{\varepsilon}|f|^{2} d x+\int_{\varepsilon}^{1}|f|^{2} d x\right)^{1 / 2} .
$$

In what concerns the corresponding fractional spaces, it is easy to see that $X_{0}^{\varepsilon} \equiv H_{0}^{1}(-1,1)$ with the usual norm and that $X_{-1 / 2}^{\varepsilon} \equiv\left(L^{2}(-1,1),|\cdot|_{\varepsilon}\right)$.

The conservation of the energy $E^{\varepsilon}$ in (1.5) corresponds to the fact that system (1.2) generates a group of isometries in $X_{0}^{\varepsilon} \times X_{-1 / 2}^{\varepsilon}$, i.e.

$$
\begin{aligned}
\left\|U^{\varepsilon}(t)\right\|_{X_{0}^{\varepsilon} \times X_{-1 / 2}^{\varepsilon}}^{2} & =\sum_{k \in \mathbb{Z} \backslash\{0\}}\left[\left|a_{k} e^{i \alpha_{k}^{\varepsilon} t}\right|^{2}+\left|b_{k} e^{i \beta_{k}^{\varepsilon} t}\right|^{2}\right] \\
& =\sum_{k \in \mathbb{Z} \backslash\{0\}}\left[\left|a_{k}\right|^{2}+\left|b_{k}\right|^{2}\right]=\left\|\left(u_{0}, u_{1}\right)\right\|_{X_{0}^{\varepsilon} \times X_{-1 / 2}^{\varepsilon}}^{2} .
\end{aligned}
$$

By analogy with the quantity $\delta_{k}$ measuring the distance between consecutive eigenvalues in the limit problem we introduce

$$
\delta_{k}^{\varepsilon}=\beta_{k+\sigma_{k}}^{\varepsilon}-\alpha_{k}^{\varepsilon} .
$$

As we have shown in Theorem 3.1,

$$
\inf _{k \in \mathbb{Z} \backslash\{0\}}\left|\delta_{k}^{\varepsilon}\right| \sim \sqrt{\varepsilon} .
$$


We now introduce the Hilbert space $H^{\varepsilon}=\left(X_{0}^{\varepsilon} \times X_{-1 / 2}^{\varepsilon},\|\cdot\|_{\varepsilon}\right)$ the norm $\|\cdot\|_{\varepsilon}$ being as follows: If $U=\sum_{k \in \mathbb{Z} \backslash\{0\}}\left(a_{k} \bar{\phi}_{k}^{\varepsilon}+b_{k} \bar{\psi}_{k}^{\varepsilon}\right)$ then

$$
\|U\|_{\varepsilon}^{2}=\left|b_{-1}\right|^{2}+\left|b_{1}\right|^{2}+\sum_{k \in \mathbb{Z} \backslash\{0\}}\left|a_{k}\right|^{2}+\sum_{k \in \mathbb{Z} \backslash\{0, \pm 1\}} \frac{\left|a_{k-\sigma_{k}}+b_{k}\right|^{2}}{\left(\delta_{k-\sigma_{k}}^{\varepsilon}\right)^{2}} .
$$

The algebraic structure of the norm $\|\cdot\|_{\varepsilon}$ is similar to the asymmetric norm $\|\cdot\|_{H}$ introduced in (5.9) for the limit problem. However, in view of (6.4) the norm $\|\cdot\|_{\varepsilon}$ is equivalent to the usual $H_{0}^{1}(-1,1) \times L^{2}(-1,1)$-norm.

The first result of this section is as follows:

Theorem 6.1. There exists $C>0$ independent of $0<\varepsilon<1$ such that the solutions $U^{\varepsilon}=\left(u^{\varepsilon}, u_{t}^{\varepsilon}\right)$ of (1.2) satisfy

$$
\left\|U^{\varepsilon}(t)\right\|_{\varepsilon}^{2} \leq C\left(1+t^{2}\right)\left\|U_{0}\right\|_{\varepsilon}, \forall t \geq 0
$$

for all $U_{0}=\left(u_{0}, u_{1}\right) \in H_{0}^{1}(-1,1) \times L^{2}(-1,1)$ and $0<\varepsilon<1$.

Proof. We have

$$
\begin{aligned}
& \left\|U^{\varepsilon}(t)\right\|_{\varepsilon}^{2}=\left|b_{-1} e^{i \beta_{-1}^{\epsilon} t}\right|^{2}+\left|b_{1} e^{i \beta_{1}^{\epsilon} t}\right|^{2}+\sum_{k \in \mathbb{Z} \backslash\{0\}}\left|a_{k} e^{i \alpha_{k}^{\varepsilon} t}\right|^{2} \\
& +\sum_{k \in \mathbb{Z} \backslash\{0, \pm 1\}} \frac{\left|a_{k-\sigma_{k}} e^{i \alpha_{k-\sigma_{k}}^{\epsilon} t}+b_{k} e^{i \beta_{k}^{\varepsilon} t}\right|^{2}}{\left(\delta_{k-\sigma_{k}}^{\varepsilon}\right)^{2}} \\
& \leq\left|b_{-1}\right|^{2}+\left|b_{1}\right|^{2}+\sum_{k \in \mathbb{Z} \backslash\{0\}}\left|a_{k}\right|^{2} \\
& \quad+2 \sum_{k \in \mathbb{Z} \backslash\{0, \pm 1\}}\left(\frac{\left|a_{k-\sigma_{k}}+b_{k}\right|^{2}}{\left(\delta_{k-\sigma_{k}}^{\varepsilon}\right)^{2}}+\left|b_{k}\right|^{2} \frac{\mid e^{i \alpha_{k-\sigma_{k}}^{\epsilon}-\left.e^{i \beta_{k}^{\varepsilon} t}\right|^{2}}}{\left(\delta_{k-\sigma_{k}}^{\varepsilon}\right)^{2}}\right) \\
& \leq C\left(1+t^{2}\right)\left\|U_{0}\right\|_{\varepsilon}^{2}
\end{aligned}
$$

since

$$
\frac{\left|e^{i \alpha_{k-\sigma_{k}}^{\varepsilon} t}-e^{i \beta_{k}^{\varepsilon} t}\right|^{2}}{\left(\delta_{k-\sigma_{k}}^{\varepsilon}\right)^{2}} \leq t^{2}
$$

and

$\left|b_{k}\right|^{2} \leq 2\left(\left|a_{k-\sigma_{k}}\right|^{2}+\left|a_{k-\sigma_{k}}+b_{k}\right|^{2}\right) \leq C\left(\left|a_{k-\sigma_{k}}\right|^{2}+\frac{\left|a_{k-\sigma_{k}}+b_{k}\right|^{2}}{\left(\delta_{k-\sigma_{k}}^{\varepsilon}\right)^{2}}\right)$.

REMARK 6.2. Theorem 6.1 is the analogue of Theorem 5.1 in the context of the approximate systems (1.2).

However it is not clear whether Theorem 5.1 is a consequence of passing to the limit as $\varepsilon \rightarrow 0$ in Theorem 6.1 . Indeed, given $U_{0}$ in the asymmetric space $H$ it is not clear if the norms of $U_{0}$ in $H_{\varepsilon}$ remain bounded or not.

Esaim: CoCv, July 1997, Vol.2, PP. 231-280 
In order to solve this problem we need some further results on how solutions of (1.2) approximate the solutions of the limit system.

Let us consider $U_{0}=\left(u_{0}, u_{1}\right) \in H_{0}^{1}(-1,1) \times L^{2}(-1,1)$ such that

$$
\begin{aligned}
& \frac{1}{2 \varepsilon} \int_{-\varepsilon}^{\varepsilon}\left|u_{1}(x)\right|^{2} d x \leq C, \forall 0<\varepsilon<1 \\
& \frac{1}{2 \varepsilon} \int_{-\varepsilon}^{\varepsilon} u_{1}(x) d x \rightarrow z^{1}, \text { as } \varepsilon \rightarrow 0 .
\end{aligned}
$$

Under these assumptions the weak convergence result of Theorem 2.1 applies. Now, we can develop $U_{0}$ in Fourier series both in the basis associated to the approximate and limit systems:

$$
\begin{aligned}
U_{0} & =\sum_{k \in \mathbb{Z} \backslash\{0\}}\left(a_{k}^{\varepsilon} \overline{\phi_{k}^{\varepsilon}}+b_{k}^{\varepsilon} \overline{\psi_{k}^{\varepsilon}}\right) \\
U_{0} & =\sum_{k \in \mathbb{Z} \backslash\{0\}}\left(a_{k} \overline{\phi_{k}}+b_{k} \overline{\psi_{k}}\right) .
\end{aligned}
$$

Note that in $(6.10), U_{0} \equiv\left(u_{0}, u_{1}, z_{1}\right) \in X_{0} \times X_{-1 / 2}$.

We also introduce

$$
c(\varepsilon)=\frac{1}{2 \varepsilon} \int_{-\varepsilon}^{\varepsilon} u^{1}(x) d x-z^{1} .
$$

We have the following result:

LEMMA 6.3. There exists $C>0$ such that

$$
\begin{aligned}
&\left|a_{k}^{\varepsilon}-a_{k}\right| \leq C(c(\varepsilon)+k \sqrt{\varepsilon}), \forall k:|k| \leq \delta \varepsilon^{-1 / 6} \\
&\left|b_{k}^{\varepsilon}-b_{k}\right| \leq C(c(\varepsilon)+k \sqrt{\varepsilon}), \forall k:|k| \leq \delta \varepsilon^{-1 / 6}
\end{aligned}
$$

with $\delta>0$ small enough independent of $0<\varepsilon<1$.

Proof. We focus on the second estimate, the first one being easier to obtain.

From Theorems 4.7 and 4.9 on the convergence of the eigenvalues and eigenfunctions we have:

$$
\begin{aligned}
& \left|b_{k}-b_{k}^{\varepsilon}\right|=\left|<U_{0}, \bar{\psi}_{k}>_{X_{0} \times X_{1 / 2}}-<U_{0}, \bar{\psi}_{k}^{\varepsilon}>_{X_{0}^{\varepsilon} \times X_{-1 / 2}^{\varepsilon}}\right| \\
& \leq\left|<u_{0}, \psi_{k}-\psi_{k}^{\varepsilon}>_{H_{0}^{1}(-1,1)}\right|+\mid \int_{-1}^{1} u_{1} \omega_{k} \psi_{k} d x+z_{1} \omega_{k} \psi_{k}(0) \\
& \quad-\int_{\varepsilon<|x|<1} u_{1} \beta_{k}^{\varepsilon} \psi_{k}^{\varepsilon}-\frac{1}{2 \varepsilon} \int_{-\varepsilon}^{\varepsilon} u_{1} \beta_{k}^{\varepsilon} \psi_{k}^{\varepsilon} \mid \\
& \leq\left\|u_{0}\right\|_{H_{0}^{1}(-1,1)}\left\|\psi_{k}-\psi_{k}^{\varepsilon}\right\|_{H_{0}^{1}(-1,1)}+\left\|u_{1}|| L_{L^{2}}\right\| \omega_{k} \psi_{k}-\beta_{k}^{\varepsilon} \psi_{k}^{\varepsilon} \|_{L^{2}} \\
& +\frac{1}{2 \varepsilon}\left|\int_{-\varepsilon}^{\varepsilon}\left(z_{1} \omega_{k} \psi_{k}(0)-u_{1} \beta_{k}^{\varepsilon} \psi_{k}^{\varepsilon}(x)\right)\right|+\left|\int_{|x|<\varepsilon} u_{1} \beta_{k}^{\varepsilon} \psi_{k}^{\varepsilon}\right| .
\end{aligned}
$$

By Theorems 4.7 and 4.9 the first two terms are (uniformly) of the order of $\sqrt{\varepsilon}$. The last term can be estimated easily as follows

$$
\begin{aligned}
\left|\int_{|x|<\varepsilon} u_{1} \beta_{k}^{\varepsilon} \psi_{k}^{\varepsilon}\right| & \leq\left\|u_{1}\right\|_{L^{2}(-1,1)} \sqrt{2 \varepsilon}\left(\frac{1}{2 \varepsilon} \int_{-\varepsilon}^{\varepsilon}\left|\beta_{k}^{\varepsilon} \psi_{k}^{\varepsilon}\right|^{2}\right)^{1 / 2} \\
& \leq \sqrt{2 \varepsilon}\left\|u_{1}\right\|_{L^{2}(-1,1)} .
\end{aligned}
$$


Let us analyze the third term. We have,

$$
\begin{aligned}
& \left|\frac{1}{2 \varepsilon} \int_{-\varepsilon}^{\varepsilon}\left(z_{1} \omega_{k} \psi_{k}(0)-u_{1} \beta_{k}^{\varepsilon} \psi_{k}^{\varepsilon}(x)\right)\right| \leq \frac{1}{2 \varepsilon}\left|\int_{-\varepsilon}^{\varepsilon}\left(u_{1}-z_{1}\right)\right| \omega_{k} \psi_{k}(0) \\
& \quad+\frac{1}{2 \varepsilon} \int_{-\varepsilon}^{\varepsilon}\left|u_{1} \psi_{k}(0)\left(\omega_{k}-\beta_{k}^{\varepsilon}\right)\right|+\frac{1}{2 \varepsilon} \int_{-\varepsilon}^{\varepsilon}\left|u_{1} \beta_{k}^{\varepsilon}\left(\psi_{k}(0)-\psi_{k}^{\varepsilon}(0)\right)\right| \\
& \quad+\frac{1}{2 \varepsilon} \int_{-\varepsilon}^{\varepsilon}\left|u_{1} \beta_{k}^{\varepsilon}\left(\psi_{k}^{\varepsilon}(0)-\psi_{k}^{\varepsilon}(x)\right)\right| \\
& \quad \leq c(\varepsilon)\left|\omega_{k} \psi_{k}(0)\right|+C\left|\psi_{k}(0)\right|\left|\omega_{k}-\beta_{k}^{\varepsilon}\right|+C\left|\beta_{k}^{\varepsilon}\right|\left|\psi_{k}(0)-\psi_{k}^{\varepsilon}(0)\right| \\
& \quad+\frac{1}{2 \varepsilon} \int_{-\varepsilon}^{\varepsilon}\left|u_{1} \beta_{k}^{\varepsilon}\left(\psi_{k}(0)-\psi_{k}^{\varepsilon}(x)\right)\right| .
\end{aligned}
$$

The first two terms can be bounded by $c(\varepsilon)+\sqrt{\varepsilon}$ while the third has to be majorized by $k \sqrt{\varepsilon}$. Finally, the last term can be estimated as follows:

$$
\begin{aligned}
& \frac{1}{2 \varepsilon} \int_{-\varepsilon}^{\varepsilon}\left|u_{1} \beta_{k}^{\varepsilon}\left(\psi_{k}(0)-\psi_{k}^{\varepsilon}(x)\right)\right| d x \\
& \quad \leq\left|\beta_{k}^{\varepsilon}\right|\left(\frac{1}{2 \varepsilon} \int_{-\varepsilon}^{\varepsilon}\left|u_{1}\right|^{2}\right)^{1 / 2}\left(\frac{1}{2 \varepsilon} \int_{-\varepsilon}^{\varepsilon}\left|\psi_{k}(x)-\psi_{k}(0)\right|^{2}\right)^{1 / 2} \\
& \quad \leq C\left|\beta_{k}^{\varepsilon}\right| \sqrt{\varepsilon}\left\|\psi_{k}\right\|_{H_{0}^{1}(-1,1)} \leq C \sqrt{\varepsilon k}
\end{aligned}
$$

since $\left|\beta_{k}^{\varepsilon}\right| \leq C k$. This concludes the proof of the Lemma.

Theorem 6.4. Let $U_{0} \in H$ be such that (6.7)-(6.8) hold. Define

$$
U_{0}^{\varepsilon}=\sum_{|k| \leq K(\varepsilon)}\left(a_{k}^{\varepsilon} \overline{\phi_{k}^{\varepsilon}}+b_{k}^{\varepsilon} \overline{\psi_{k}^{\varepsilon}}\right)
$$

with $K(\varepsilon)=\min \left(c(\varepsilon)^{-2 / 3+\gamma}, \delta \varepsilon^{-1 / 6}\right)$ for any $\gamma>0$ with $\delta>0$ small enough, where $\left\{a_{k}^{\epsilon}, b_{k}^{\epsilon}\right\}$ are the Fourier coefficients of $U_{0}$ in the basis of $\left\{\bar{\phi}_{k}^{\varepsilon}, \bar{\psi}_{k}^{\varepsilon}\right\}$. Then,

$$
\left\|U_{0}^{\varepsilon}\right\|_{\varepsilon} \rightarrow\left\|U_{0}\right\|_{H}, \text { as } \varepsilon \rightarrow 0
$$

REMARK 6.5. Theorem 6.4 states that the norm $\left\|U_{0}\right\|_{H}$ can be obtained as the limit of the norms $\left\|U_{0}^{\varepsilon}\right\|_{\varepsilon}$ provided $U_{0}^{\varepsilon}$ is a suitable truncation of the Fourier series associated to $U_{0}$ in the basis $\left\{\overline{\phi_{k}^{\varepsilon}}\right\} \cup\left\{\overline{\psi_{k}^{\varepsilon}}\right\}$. 
Proof. We have

$$
\begin{aligned}
& \left|\left\|U_{0}^{\varepsilon}\right\|_{\varepsilon}^{2}-\left\|U_{0}\right\|_{H}^{2}\right|=\left.|| b_{-1}^{\varepsilon}\right|^{2}-\left|b_{-1}\right|^{2}+\left|b_{1}^{\varepsilon}\right|^{2}-\left|b_{1}\right|^{2}+\sum_{|k| \leq K(\varepsilon)}\left|a_{k}^{\varepsilon}\right|^{2} \\
& \quad-\sum_{k \in \mathbb{Z} \backslash\{0\}}\left|a_{k}\right|^{2}+\sum_{\substack{|k| \leq K(\varepsilon) \\
k \neq \pm 1}} \frac{\left|a_{k-\sigma_{k}}^{\varepsilon}+b_{k}^{\varepsilon}\right|^{2}}{\left(\delta_{k-\sigma_{k}}^{\varepsilon}\right)^{2}}-\sum_{k \in \mathbb{Z} \backslash\{0, \pm 1\}} \frac{\left|a_{k-\sigma_{k}}+b_{k}\right|^{2}}{\delta_{k-\sigma_{k}}^{2}} \mid \\
& \leq C\left(\left\|U_{0}\right\| H+\left\|U_{0}^{\varepsilon}\right\|_{\varepsilon}\right) K(\varepsilon)(c(\varepsilon)+K(\varepsilon) \sqrt{\varepsilon})+\sum_{|k|>K(\varepsilon)}\left|a_{k}\right|^{2} \\
& \quad+\sum_{|k|>K(\varepsilon)} \frac{\left|a_{k-\sigma_{k}}+b_{k}\right|^{2}}{\left(\delta_{k-\sigma_{k}}\right)^{2}}+\mid \sum_{\substack{|k| \leq K(\varepsilon) \\
k \neq \pm 1}}\left[\frac{\left|a_{k-\sigma_{k}}^{\varepsilon}+b_{k}^{\varepsilon}\right|^{2}}{\left(\delta_{k-\sigma_{k}}^{\varepsilon}\right)^{2}}-\frac{\left|a_{k-\sigma_{k}}+b_{k}\right|^{2}}{\left(\delta_{k-\sigma_{k}}\right)^{2}}\right] .
\end{aligned}
$$

The coefficient $K(\varepsilon)(C(\varepsilon)+K(\varepsilon) \sqrt{\varepsilon})$ as well as the second and third terms of the right hand side of (6.16) converge to zero as $\varepsilon \rightarrow 0$.

Concerning the last term, we have

$$
\begin{aligned}
& \left|\sum_{\substack{|k| \leq K(\varepsilon) \\
k \neq \pm 1}}\left[\frac{\left|a_{k-\sigma_{k}}^{\varepsilon}+b_{k}^{\varepsilon}\right|^{2}}{\left(\delta_{k-\sigma_{k}}^{\varepsilon}\right)^{2}}-\frac{\left|a_{k-\sigma_{k}}+b_{k}\right|^{2}}{\delta_{k-\sigma_{k}}^{2}}\right]\right| \\
& \leq \sum_{\substack{|k| \leq K(\varepsilon) \\
k \neq \pm 1}} \frac{|| a_{k-\sigma_{k}}^{\varepsilon}+\left.b_{k}^{\varepsilon}\right|^{2}-\left|a_{k-\sigma_{k}}+b_{k}\right|^{2} \mid}{\left(\delta_{k-\sigma_{k}}^{\varepsilon}\right)^{2}} \\
& +\sum_{\substack{|k| \leq K(\varepsilon) \\
k \neq \pm 1}}\left|\frac{1}{\left(\delta_{k-\sigma_{k}}^{\varepsilon}\right)^{2}}-\frac{1}{\delta_{k-\sigma_{k}}^{2}}\right|\left|a_{k-\sigma_{k}}+b_{k}\right|^{2} .
\end{aligned}
$$

We have

$$
\left|\frac{1}{\left(\delta_{k-\sigma_{k}}^{\varepsilon}\right)^{2}}-\frac{1}{\delta_{k-\sigma_{k}}^{2}}\right|=\frac{\left|\delta_{k-\sigma_{k}}^{\varepsilon}+\delta_{k-\sigma_{k}}\right|}{\left(\delta_{k-\sigma_{k}}^{\varepsilon}\right)^{2} \delta_{k-\sigma_{k}}^{2}}\left|\delta_{k-\sigma_{k}}^{\varepsilon}-\delta_{k-\sigma_{k}}\right| \leq \frac{C \sqrt{\varepsilon} K^{2}(\varepsilon)}{\delta_{k-\sigma_{k}}^{2}}
$$

since, in view of (4.8)-(4.9), $\left|\delta_{k-\sigma_{k}}^{\varepsilon}-\delta_{k-\sigma_{k}}\right| \leq C \sqrt{\varepsilon}$ and

$$
\left(\frac{1}{\delta_{k-\sigma_{k}}^{\varepsilon}}\right)^{2} \leq C K^{2}(\varepsilon) \text {. }
$$

To check that (6.19) holds we observe that

$$
\delta_{k-\sigma_{k}}^{\varepsilon} \geq \delta_{k}-C \sqrt{\varepsilon} \geq \frac{C}{K(\varepsilon)}-C \sqrt{\varepsilon} \geq \frac{C}{2 K(\varepsilon)}
$$

since $K(\varepsilon)<<\varepsilon^{-1 / 2}$. 
From (6.18) we deduce that

$$
\begin{aligned}
& \sum_{\substack{|k| \leq K(\varepsilon) \\
k \neq \pm 1}}\left|\frac{1}{\left(\delta_{k-\sigma_{k}}^{\varepsilon}\right)^{2}}-\frac{1}{\delta_{k-\sigma_{k}}^{2}}\right|\left|a_{k-\sigma_{k}}-b_{k}\right|^{2} \\
& \leq C \sqrt{\varepsilon} K^{2}(\varepsilon) \sum_{\substack{|k| \leq K(\varepsilon) \\
k \neq \pm 1}} \frac{\left|a_{k-\sigma_{k}}+b_{k}\right|^{2}}{\delta_{k-\sigma_{k}}^{2}} \leq C \sqrt{\varepsilon} K^{2}(\varepsilon)\left\|U^{0}\right\|_{H}^{2}
\end{aligned}
$$

which tends to zero as $\varepsilon \rightarrow 0$.

Finally, in view of $(6.12),(6.13)$ and $(6.19)$ we have

$$
\begin{aligned}
& \sum_{\substack{|k| \leq K(\varepsilon) \\
k \neq \pm 1}} \frac{|| a_{k-\sigma_{k}}^{\varepsilon}+\left.b_{k}^{\varepsilon}\right|^{2}-\left|a_{k-\sigma_{k}}+b_{k}\right|^{2} \mid}{\left(\delta_{k-\sigma_{k}}^{\varepsilon}\right)^{2}} \\
& =\sum_{\substack{|k| \leq K(\varepsilon) \\
k \neq \pm 1}} \frac{|| a_{k-\sigma_{k}}^{\varepsilon}+b_{k}^{\varepsilon}|-| a_{k-\sigma_{k}}+b_{k}|||| a_{k-\sigma_{k}}^{\varepsilon}+b_{k}|+| a_{k-\sigma_{k}}^{\varepsilon}+b_{k}||}{\left(\delta_{k-\sigma_{k}}^{\varepsilon}\right)^{2}} \\
& \leq C(c(\varepsilon)+K(\varepsilon) \sqrt{\varepsilon}) \sum_{\substack{|k| \leq K(\varepsilon) \\
k \neq \pm 1}} \frac{\left(\left|a_{k-\sigma_{k}}^{\varepsilon}+b_{k}^{\varepsilon}\right|+\left|a_{k-\sigma_{k}}+b_{k}\right|\right)}{\left(\delta_{k-\sigma_{k}}^{\varepsilon}\right)^{2}} \\
& \leq C K(\varepsilon)(c(\varepsilon)+K(\varepsilon) \sqrt{\varepsilon}) \\
& \quad \times\left(\sum_{\substack{|k| \leq K(\varepsilon) \\
k \neq \pm 1}}\left(\frac{\left|a_{k-\sigma_{k}}^{\varepsilon}+b_{k}^{\varepsilon}\right|^{2}}{\left(\delta_{k-\sigma_{k}}^{\varepsilon}\right)^{2}}+\frac{\left|a_{k-\sigma_{k}}+b_{k}\right|^{2}}{\left(\delta_{k-\sigma_{k}}^{\varepsilon}\right)^{2}}\right)^{1 / 2}\right)^{1 / 2}(\varepsilon) \\
& \leq C K^{3 / 2}(\varepsilon)(c(\varepsilon)+K(\varepsilon) \sqrt{\varepsilon})\left(\left\|U_{0}^{\varepsilon}\right\|_{\varepsilon}^{2}+\left\|U_{0}\right\|_{H}^{2}\right)^{1 / 2} .
\end{aligned}
$$

Clearly, the coefficient $K^{3 / 2}(\varepsilon)(c(\varepsilon)+K(\varepsilon) \sqrt{\varepsilon})$ tends to zero as $\varepsilon \rightarrow 0$.

Combining $(6.16),(6.17),(6.20)$ and $(6.21)$ we have

$$
\left|\left\|U_{0}^{\varepsilon}\right\|_{\varepsilon}^{2}-\left\|U_{0}\right\|_{H}^{2}\right| \leq o(1)\left(1+\left\|U_{0}^{\varepsilon}\right\|_{\varepsilon}+\left\|U_{0}\right\|_{H}\right) \text { as } \varepsilon \rightarrow 0
$$

and this implies $(6.15)$.

From these results we can recover the well-posedness of the limit system in the asymmetric space $H$.

Corollary 6.6. As a consequence of Theorem 6.1 and the approximation results above, the limit system (1.1) is well-posed in the asymmetric space $H$.

Proof. We consider first the case of initial data $U_{0} \in H$ such that the conditions (6.7) and (6.8) hold, and obtain the stability result (5.13). The general case $U_{0} \in H$ can be then easily obtained by a density argument.

Given $U_{0} \in H$ satisfying (6.7) and (6.8) we define $U_{0}^{\varepsilon}$ as in (6.14). Let $U^{\varepsilon}(t)$ be the solution of $(1.2)$ with data $U_{0}^{\varepsilon}$. By theorem 6.1 we have

$$
\left\|U^{\varepsilon}(t)\right\|_{\varepsilon}^{2} \leq C\left(1+t^{2}\right)\left\|U_{0}^{\varepsilon}\right\|_{\varepsilon}^{2} .
$$


Taking in (6.22) the lim inf as $\varepsilon \rightarrow 0$, using Fatou's Lemma and Theorem 6.4 we deduce that the solution $U$ of (1.1) satisfies

$\|U(t)\|_{H}^{2} \leq \liminf _{\varepsilon \rightarrow 0}\left\|U^{\varepsilon}(t)\right\|_{\varepsilon}^{2} \leq C\left(1+t^{2}\right) \liminf _{\varepsilon \rightarrow 0}\left\|U_{0}^{\varepsilon}\right\|_{\varepsilon}^{2}=C\left(1+t^{2}\right)\left\|U_{0}\right\|_{H}^{2}$ and this concludes the proof of the corollary.

\section{Further CONVERGEnCE RESUlts}

The techniques of section 6 allow us to obtain the following result about the convergence of the solutions of (1.2) towards the solution of (1.1).

Consider $\left(u_{0}, u_{1}, z_{1}\right) \in H_{0}^{1}(-1,1) \times L^{2}(-1,1) \times \mathbb{R}$ such that

$$
\begin{gathered}
\frac{1}{2 \varepsilon} \int_{-\varepsilon}^{\varepsilon}\left|u_{1}\right|^{2} d x \leq C \\
\frac{1}{2 \varepsilon} \int_{-\varepsilon}^{\varepsilon} u_{1} \rightarrow z_{1} \text { as } \varepsilon \rightarrow 0 .
\end{gathered}
$$

Let $u$ be the solution of the limit problem (1.1) and $\left\{u^{\varepsilon}\right\}_{\varepsilon>0}$ the sequence of solutions of (1.2) with the same initial data. Let us also define the truncated family of solutions of (1.2):

$$
\widetilde{u}^{\varepsilon}=\sum_{|k| \leq K(\varepsilon)}\left(a_{k}^{\varepsilon} \phi_{k}^{\varepsilon} e^{i \alpha_{k}^{\varepsilon} t}+b_{k}^{\varepsilon} \psi_{k}^{\varepsilon} e^{i \beta_{k}^{\varepsilon} t}\right)
$$

with $K(\varepsilon)$ as in Theorem 6.4 .

We have the following

Theorem 7.1. Under the assumptions above, for any $T>0$ we have

$$
\left\{\begin{array}{l}
\widetilde{u}^{\varepsilon} \rightarrow u \text { in } C\left([0, T] ; H_{0}^{1}(-1,1)\right) \cap C^{1}\left([0, T] ; L^{2}(-1,1)\right) \\
\frac{1}{2 \varepsilon} \int_{-\varepsilon}^{\varepsilon} \widetilde{u}_{t}^{\varepsilon} d x \rightarrow u_{t}(0, t) \text { in } C([0, T]), \text { as } \varepsilon \rightarrow 0 .
\end{array}\right.
$$

If moreover,

$$
\frac{1}{2 \varepsilon} \int_{-\varepsilon}^{\varepsilon}\left|u_{1}\right|^{2} d x \rightarrow\left(z_{1}\right)^{2} \text { as } \varepsilon \rightarrow 0
$$

then

$$
\left\{\begin{array}{l}
u^{\varepsilon} \rightarrow u \text { in } C\left([0, T] ; H_{0}^{1}(-1,1)\right) \cap C^{1}\left([0, T] ; L^{2}(-1,1)\right) \\
\frac{1}{2 \varepsilon} \int_{-\varepsilon}^{\varepsilon} u_{t}^{\varepsilon} d x \rightarrow u_{t}(0, t) \text { in } C([0, T]), \text { as } \varepsilon \rightarrow 0
\end{array}\right.
$$

REMARK 7.2. The first part of Theorem 7.1 ensures that, under the conditions in which $u^{\varepsilon}$ converges weakly to $u$ in the energy space (see Theorem 2.1) the truncated sequence $\widetilde{u^{\varepsilon}}$ converges strongly.

The second part asserts, in the case in which the strong convergence holds (see Theorem 2.3), that the convergence holds uniformly in time and not only in $L^{2}$ with respect to time.

Proof. Let us proof the first statement. By $\widetilde{U}^{\varepsilon}$ we denote the vector-valued unknown

$$
\widetilde{U}^{\varepsilon}=\left(u^{\varepsilon}, u_{t}^{\varepsilon}, \frac{1}{2 \varepsilon} \int_{-\varepsilon}^{\varepsilon} u_{t}^{\varepsilon} d x\right)
$$

and $U(x, t)=\left(u(x, t), u_{t}(x, t), u_{t}(0, t)\right)$. 
To simplify notation we will denote the norm $\|\cdot\|_{H_{0}^{1}(-1,1) \times\left(L^{2}(-1,1) \times \mathbb{R}\right)}$ by $\|\cdot\|$ along the proof. We have

$$
\begin{aligned}
& \left\|U(t)-\tilde{U}^{\varepsilon}(t)\right\|_{H_{0}^{1}(-1,1) \times\left(L^{2}(-1,1) \times \mathbb{R}\right)} \\
& =\left\|\sum_{|k| \leq K(\varepsilon)}\left[\left(a_{k} \bar{\phi}_{k} e^{i k \pi t}+b_{k} \bar{\psi}_{k} e^{i \omega_{k} t}\right)-\left(a_{k}^{\varepsilon} \bar{\phi}_{k}^{\varepsilon} e^{i \alpha_{k}^{\varepsilon} t}+b_{k}^{\varepsilon} \bar{\psi}_{k}^{\varepsilon} e^{i \beta_{k}^{\varepsilon} t}\right)\right]\right\| \\
& \quad+\left\|\sum_{|k|>K(\varepsilon)}\left(a_{k} \bar{\phi}_{k} e^{i k \pi t}+b_{k} \bar{\psi}_{k} e^{i \omega_{k} t}\right)\right\|
\end{aligned}
$$

Clearly

$$
\left\|\sum_{|k|>K(\varepsilon)}\left(a_{k} \bar{\phi}_{k} e^{i k \pi t}+b_{k} \bar{\psi}_{k} e^{i \omega_{k} t}\right)\right\|=\left(\sum_{|k|>K(\varepsilon)}\left(\left|a_{k}\right|^{2}+\left|b_{k}\right|^{2}\right)\right)^{1 / 2}
$$

and this tends to zero as $\varepsilon \rightarrow 0$.

On the other hand,

$$
\begin{aligned}
& \left\|\sum_{|k|<K(\varepsilon)}\left(a_{k} \bar{\phi}_{k} e^{i k \pi t}-a_{k}^{\varepsilon} \bar{\phi}_{k}^{\varepsilon} e^{i \alpha_{k}^{\varepsilon} t}\right)\right\|^{2} \\
& \leq C \sum_{|k| \leq K(\varepsilon)}\left[\left|a_{k}-a_{k}^{\varepsilon}\right|^{2}+\left|a_{k}^{\varepsilon}\right|^{2}\left|e^{i k \pi t}-e^{i \alpha_{k}^{\varepsilon} t}\right|^{2}\right] \\
& \quad+C\left\|\sum_{|k| \leq K(\varepsilon)} a_{k}^{\varepsilon}\left(\bar{\phi}_{k}-\bar{\phi}_{k}^{\varepsilon}\right) e^{i k \pi t}\right\|^{2} .
\end{aligned}
$$

By Lemma 6.3 we have

$$
\sum_{|k| \leq K(\varepsilon)}\left|a_{k}-a_{k}^{\varepsilon}\right|^{2} \leq C K(\varepsilon)(c(\varepsilon)+K(\varepsilon) \sqrt{\varepsilon})^{2}
$$

and, in view of the choice of $K(\varepsilon)$, this converges to zero as $\varepsilon \rightarrow 0$.

By Theorem 4.1 we also have,

$$
\left|e^{i k \pi t}-e^{i \alpha_{k}^{\varepsilon} t}\right| \leq\left|k \pi-\alpha_{k}^{\varepsilon}\right| t \leq C \sqrt{\varepsilon} T, \forall|k| \leq K(\varepsilon), \forall t \in[0, T] .
$$

Therefore, the second term on the right hand side of (7.9) converges to zero too as $\varepsilon \rightarrow 0$.

To estimate the last term we observe that, in view of Theorem 4.9 ,

$$
\left\|\bar{\phi}_{k}-\bar{\phi}_{k}^{\varepsilon}\right\| \leq C \sqrt{\varepsilon}+\left|\alpha_{k}^{\varepsilon}\right| \int_{-\varepsilon}^{\varepsilon} \phi_{k}^{\varepsilon} d x \leq C \sqrt{\varepsilon}(1+K(\varepsilon)) .
$$


Thus

$$
\begin{aligned}
& \left\|\sum_{|k| \leq K(\varepsilon)} a_{k}^{\varepsilon}\left(\bar{\phi}_{k}-\bar{\phi}_{k}^{\varepsilon}\right) e^{i k \pi t}\right\| \leq C \sqrt{\varepsilon}(1+K(\varepsilon)) \sum_{|k| \leq K(\varepsilon)}\left|a_{k}^{\varepsilon}\right| \\
& \leq C \sqrt{\varepsilon}(1+K(\varepsilon)) K^{1 / 2}(\varepsilon)\left(\sum_{|k| \leq K(\varepsilon)}\left|a_{k}^{\varepsilon}\right|^{2}\right)^{1 / 2}
\end{aligned}
$$

which tends to zero too.

The term

$$
\left\|\sum_{|k| \leq K(\varepsilon)}\left(b_{k} \bar{\psi}_{k} e^{i \omega_{k} t}-b_{k}^{\varepsilon} e^{i \beta_{k}^{\varepsilon} t}\right)\right\|_{H_{0}^{1}(-1,1) \times\left(L^{2}(-1,1) \times \mathbb{R}\right)}
$$

can be estimated in a similar way.

Let us prove now the second part of the theorem. In view of the first one it is sufficient to check that

$$
\left\|\sum_{|k|>K(\varepsilon)} a_{k}^{\varepsilon} \bar{\phi}_{k}^{\varepsilon} e^{i \alpha_{k}^{\varepsilon} t}+b_{k}^{\varepsilon} \bar{\psi}_{k}^{\varepsilon} e^{i \beta_{k}^{\epsilon} t}\right\|_{H_{0}^{1}(-1,1) \times\left(L^{2}(-1,1) \times \mathbb{R}\right)}
$$

converges uniformly to zero as $\varepsilon \rightarrow 0$.

The norm of the first two components can be bounded above by the norms in $X_{0}^{\varepsilon} \times X_{-1 / 2}^{\varepsilon}$ which coincide with

$$
\left(\sum_{|k|>K(\varepsilon)}\left|a_{k}^{\varepsilon}\right|^{2}+\left|b_{k}^{\varepsilon}\right|^{2}\right)^{1 / 2}
$$

and this tends to zero as $\varepsilon \rightarrow 0$ since

$$
\sum_{k \in \mathbb{Z} \backslash\{0\}}\left|a_{k}^{\varepsilon}\right|^{2}+\left|b_{k}^{\varepsilon}\right|^{2}=2 E^{\varepsilon}(0) \rightarrow 2 E(0)=\sum_{k \in \mathbb{Z} \backslash\{0\}}\left(\left|a_{k}\right|^{2}+\left|b_{k}\right|^{2}\right)
$$

in view of the assumptions on the initial data which guarantee the convergence of the energies.

Concerning the third component we have

$$
\begin{gathered}
\left|\sum_{|k|>K(\varepsilon)} \frac{b_{k}^{\varepsilon} \beta_{k}^{\varepsilon}}{2 \varepsilon} \int_{-\varepsilon}^{\varepsilon} \psi_{k}^{\varepsilon} d x\right| \leq \frac{1}{\sqrt{2 \varepsilon}}\left(\int_{-\varepsilon}^{\varepsilon}\left(\sum_{|k|>K(\varepsilon)} b_{k}^{\varepsilon} \beta_{k}^{\varepsilon} \psi_{k}^{\varepsilon}\right)^{2}\right)^{1 / 2} \\
\leq\left\|\sum_{|k|>K(\varepsilon)} b_{k}^{\varepsilon} \beta_{k}^{\varepsilon} \psi_{k}^{\varepsilon}\right\|_{X_{-1 / 2}^{\varepsilon}}=\left(\sum_{|k|>K(\varepsilon)}\left|b_{k}^{\varepsilon} \beta_{k}^{\varepsilon}\right|^{2}\right)^{1 / 2}
\end{gathered}
$$

which tends to zero too.

This concludes the proof of Theorem 7.1. 


\section{Controllability}

Let us consider the problem of controlling the dynamics of the limit system by means of a control $v=v(t)$ acting on the extreme $x=1$.

The equations of motion read now as follows:

$$
\begin{cases}u_{t t}=u_{x x}, & \text { for }-1<x<0,0<t<T \\ u_{t t}=u_{x x}, & \text { for } 0<x<1,0<t<T \\ u\left(0^{+}, t\right)=u\left(0^{-}, t\right)=z(t), & \text { for } 0<t<T \\ M z_{t t}(t)=u_{x}\left(0^{+}, t\right)-u_{x}\left(0^{-}, t\right), & \text { for } 0<t<T \\ u(-1, t)=0, & \text { for } 0<t<T \\ u(1, t)=v(t), & \text { for } 0<t<T .\end{cases}
$$

The only difference between system (8.1) and the original uncontrolled system (1.1) is that we have replaced the homogeneous boundary condition $u(1, t)=0$ by $u(1, t)=v(t)$.

The control time $T>0$ is fixed a priori. Due to the finite speed of propagation it is natural to assume that $T>4$.

The control problem can be formulated as follows: we assume that the control $v$ belongs to $L^{2}(0, T)$. The question consists in characterizing the space of controllable initial data $\left(u_{0}, u_{1}, z_{1}\right)$ for which there exists a control $v \in L^{2}(0, T)$ such that the solution $u$ of $(8.1)$ taking the initial data

$$
\left\{\begin{array}{l}
u(x, 0)=u_{0}(x), u_{t}(x, 0)=u_{1}(x) \quad \text { in } \quad(-1,1) \\
u_{t}(0,0)=z_{1}=z_{t}(0)
\end{array}\right.
$$

is at rest at time $t=T$, i.e.

$$
\left\{\begin{array}{l}
u(x, T)=u_{t}(x, T)=0 \quad \text { in } \quad(-1,1) \\
z_{t}(T)=u_{t}(0, T)=0
\end{array}\right.
$$

The answer to this problem was obtained in [4]. It turns out that the sharp controllable space is an asymmetric space. We recall this result in section 8.1 below and give a complete explanation in terms of Fourier series. In section 8.2 we recover this result as the limit when $\varepsilon \rightarrow 0$ of uniform partial controllability results for the approximate systems (1.2).

\subsection{Controllability of the limit system IN THE ASYMMETRIC SPACE}

The following result was proved in [4]:

Theorem 8.1. For any $\left(u_{0}, u_{1}, z_{0}, z_{1}\right) \in L^{2}(-1,1) \times H^{-1}(-1,1) \times \mathbb{R} \times \mathbb{R}$ such that

$$
\left\{\begin{array}{l}
\left.u_{0}\right|_{(-1,0)} \in H^{1}(-1,0),\left.u_{1}\right|_{(-1,0)} \in L^{2}(-1,0) \\
u_{0}(-1)=0, u_{0}\left(0^{-}\right)=z_{0}
\end{array}\right.
$$

there exists a control $v \in L^{2}(0, T)$ such that the solution of (8.1) satisfying the initial conditions (8.2) and

$$
z(0)=z_{0}
$$

satisfies the control condition (8.3).

The map $\left(u_{0}, u_{1}, z_{0}, z_{1}\right) \rightarrow v$ is continuous in the corresponding topologies. Esaim: CoCV, July 1997, Vol.2, PP. 231-280 
Moreover, the solution $u$ of (8.1) belongs to the class

$$
\left\{\begin{array}{l}
u \in C\left([0, T] ; L^{2}(-1,1)\right) \cap C^{1}\left([0, T] ; H^{-1}(-1,1)\right) \\
z \in C^{1}([0, T]) \\
\left.u\right|_{(-1,0)} \in C\left([0, T] ; H^{1}(-1,0)\right) \cap C^{1}\left([0, T] ; L^{2}(-1,0)\right) .
\end{array}\right.
$$

Using Lions' HUM method (see [6]) it is easy to see that Theorem 8.1 can be reduced to the obtention of suitable observability inequalities for the uncontrolled system (1.1). As it was shown in [4], Theorem 8.1 is quivalent to the existence of positive constants $C_{i}>0, i=1,2$ such that

$$
\begin{aligned}
& C_{1}\left[\left\|u_{0}\right\|_{L^{2}(-1,0)}^{2}+\left\|u_{1}\right\|_{H^{-1}(-1,0)}^{2}+\left\|u_{0}\right\|_{H^{1}(0,1)}^{2}+\left\|u_{1}\right\|_{L^{2}(0,1)}^{2}+\left|z_{1}\right|^{2}\right] \\
& \leq \int_{0}^{T}\left|u_{x}(1, t)\right|^{2} d t \leq \\
& C_{2}\left[\left\|u_{0}\right\|_{L^{2}(-1,0)}^{2}+\left\|u_{1}\right\|_{H^{-1}(-1,0)}^{2}+\left\|u_{0}\right\|_{H^{1}(0,1)}^{2}+\left\|u_{1}\right\|_{L^{2}(0,1)}^{2}+\left|z_{1}\right|^{2}\right]
\end{aligned}
$$

holds for every solution of the uncontrolled system (1.1).

These inequalities assert that the quantity $\left(\int_{0}^{T}\left|u_{x}(1, t)\right|^{2} d t\right)^{1 / 2}$ defines a norm in the space of the initial data which is, roughly, equivalent to the natural norm of the asymmetric space

$$
\begin{gathered}
H_{-1}=\left\{\left(u_{0}, u_{1}, z_{1}\right) \in L^{2}(-1,1) \times H^{-1}(-1,1) \times \mathbb{R}:\left.u_{0}\right|_{(0,1)} \in H^{1}(0,1),\right. \\
\left.\left.u_{1}\right|_{(0,1)} \in L^{2}(0,1), u_{0}(1)=0\right\} .
\end{gathered}
$$

By means of the tools we have developed in section 5.3 it is easy to see that this space coincides algebraically and topologically with the following asymmetric space defined in terms of Fourier series:

$$
\begin{aligned}
H_{-1} & =\left\{U=\sum_{k \in \mathbb{Z} \backslash\{0\}}\left(a_{k} \bar{\phi}_{k}+b_{k} \bar{\psi}_{k}\right) \in X_{-1 / 2} \times X_{-1}:\|U\|_{-1}^{2}=\left|b_{-1}\right|^{2}\right. \\
& \left.+\left|b_{1}\right|^{2}+\sum_{k \in \mathbb{Z} \backslash\{0\}} \delta_{k}^{2}\left|a_{k}\right|^{2}+\sum_{k \in \mathbb{Z} \backslash\{0, \pm 1\}}\left|a_{k-\sigma_{k}}-b_{k}\right|^{2}<\infty\right\} .(8 .
\end{aligned}
$$

On the other hand, since

$$
u(x, t)=\sum_{k \in \mathbb{Z} \backslash\{0\}}\left(a_{k} e^{i k \pi t} \phi_{k}(x)+b_{k} e^{i \omega_{k} t} \psi_{k}(x)\right)
$$

we see that $(8.6)$ is equivalent to

$$
\begin{aligned}
& C_{1}\left[\left|b_{-1}\right|^{2}+\left|b_{1}\right|^{2}+\sum_{k \in \mathbb{Z} \backslash\{0\}} \delta_{k}^{2}\left|a_{k}\right|^{2}+\sum_{k \in \mathbb{Z} \backslash\{0, \pm 1\}}\left|a_{k-\sigma_{k}}-b_{k}\right|^{2}\right] \\
& \leq \int_{0}^{T}\left|\sum_{k \in \mathbb{Z} \backslash\{0\}}\left(a_{k} e^{i k \pi t} \phi_{k}^{\prime}(0)+b_{k} e^{i \omega_{k} t} \psi_{k}^{\prime}(0)\right)\right|^{2} d t \\
& \leq C_{2}\left[\left|b_{-1}\right|^{2}+\left|b_{1}\right|^{2}+\sum_{k \in \mathbb{Z} \backslash\{0\}} \delta_{k}^{2}\left|a_{k}\right|^{2}+\sum_{\substack{k \in \mathbb{Z} \backslash\{0, \pm 1\} \\
\text { Esaim: Cocv, July 1997, Vol.2, pr. 231-280 }}}\left|a_{k-\sigma_{k}}-b_{k}\right|^{2}\right] .
\end{aligned}
$$


As it was shown in [4], the fact that (8.9) holds is due precisely to the fact that the gap between consecutive eigenvalues is of the order of $\delta_{k}$ and that this quantity tends to zero as $k \rightarrow \infty$.

Indeed, it can be seen that (8.9) is a consequence of the following result by D. Ulrich [12]:

TheOrem 8.2. (D. Ulrich [12]) Let $\left\{\sigma_{n}\right\}_{n \in \mathbb{Z}}$ and $\left\{\zeta_{n}\right\}_{n \in \mathbb{Z}}$ be two sequences of distinct complex numbers such that $\sigma_{n} \neq \zeta_{n}$ for all $n$, and satisfying

$$
\left|\zeta_{n}-n\right| \rightarrow 0,\left|\sigma_{n}-n\right| \rightarrow 0 \text { as }|n| \rightarrow \infty .
$$

Then $\left\{e^{i \sigma_{n} t}\right\}_{n \in \mathbb{Z}}$ forms a Riesz basis in $L^{2}(0,2 \pi)$ and

$$
\left\{e^{i \sigma_{n} t}\right\}_{n \in \mathbb{Z}} \cup\left\{\frac{e^{i \sigma_{n} t}-e^{i \zeta_{n} t}}{\sigma_{n}-\zeta_{n}}\right\}_{n \in \mathbb{Z}}
$$

forms a Riesz basis in $L^{2}(0,4 \pi)$.

In order to obtain the controllability of the limit system as the limit when $\varepsilon \rightarrow 0$ of controllability results for the approximate systems we need a result on the theory of non-harmonic Fourier series showing, roughly, that Theorem 8.2 is stable under small perturbations of $\sigma_{n}$ and $\zeta_{n}$. This is the object of the next section.

\subsection{A NEW RESUlt in The TheOry of NOn-HARMonic Fourier series}

The main result of this section is as follows:

TheOREM 8.3. For any $0<\varepsilon<1$ we consider two sequences $\left\{\sigma_{n}^{\varepsilon}\right\}_{n \in \mathbb{Z}}$ and $\left\{\zeta_{n}^{\varepsilon}\right\}_{n \in \mathbb{Z}}$ of distinct real numbers. Then, there exists $\delta>0$ such that, if

$$
\max \left\{\left|n-\sigma_{n}^{\varepsilon}\right|,\left|n-\zeta_{n}^{\varepsilon}\right|\right\}<\delta \text { for all } n \in \mathbb{Z} \text { and } 0<\varepsilon<1,
$$

the following two properties hold:

(a) For any $0<\varepsilon<1$, the set $\left\{e^{i \sigma_{n}^{\varepsilon} t}\right\}_{n \in \mathbb{Z}} \cup\left\{\frac{e^{i \sigma_{n}^{\epsilon} t}-e^{i \zeta_{n}^{\varepsilon} t}}{\sigma_{n}^{\varepsilon}-\zeta_{n}^{\epsilon}}\right\}_{n \in \mathbb{Z}}$ constitutes a Riesz basis of $L^{2}(0,4 \pi)$.

(b) There exists a positive constant $C>0$ which is independent of $0<\varepsilon<1$, such that

$$
\begin{aligned}
\frac{1}{C} \sum_{n \in \mathbb{Z}}\left(\left|a_{n}\right|^{2}+\left|b_{n}\right|^{2}\right) & \leq \int_{0}^{4 \pi}\left|\sum_{n \in \mathbb{Z}}\left(a_{n} e^{i \sigma_{n}^{\varepsilon} t}+b_{n} \frac{\left(e^{i \sigma_{n}^{\varepsilon} t}-e^{i \zeta_{n}^{\varepsilon} t}\right)}{\sigma_{n}^{\varepsilon}-\zeta_{n}^{\varepsilon}}\right)\right|^{2} d t \\
& \leq C \sum_{n \in \mathbb{Z}}\left(\left|a_{n}\right|^{2}+\left|b_{n}\right|^{2}\right)
\end{aligned}
$$

for every $0<\varepsilon<1$ and $\left\{a_{n}\right\},\left\{b_{n}\right\} \in \ell^{2}$.

Proof. We write

$$
\sigma_{n}^{\varepsilon}=n+\lambda_{n, 0}^{\varepsilon}, \quad \zeta_{n}^{\varepsilon}=n+\lambda_{n, 1}^{\varepsilon} .
$$

In view of $(8.10)$ we know that

$$
\sup _{\substack{n \in \mathbb{Z} \\ j=0,1}}\left\{\left|\lambda_{n, j}^{\varepsilon}\right|\right\}<\delta \text { for all } 0<\varepsilon<1
$$

We need the following lemma due to D. Ulrich [12]:

Esaim: CoCV, JUly 1997, Vol.2, PP. 231-280 
LeMMA 8.4. ([12]): Let $\left\{\varphi_{n}\right\}_{n \in \mathbb{Z}}$ be a sequence of functions in $C^{2}([0,4 \pi])$ with norms less than $a>0$ for all $n \in \mathbb{Z}$. Then, for any $\left\{c_{n}\right\} \in \ell^{2}$ we have

$$
\int_{0}^{4 \pi}\left|\sum_{n \in \mathbb{Z}} c_{n} e^{i n t} \varphi_{n}(t)\right|^{2} d t \leq a^{2} M^{2} \sum_{n \in \mathbb{Z}}\left|c_{n}\right|^{2}
$$

where $M=6 \pi+M_{1} \pi^{2 / 3}, M_{1}=12 a^{2} M_{2}^{2}$ and $M_{2}=\|\psi\|_{C^{2}}$ where $\psi \epsilon$ $C^{2}(-\pi, 5 \pi)$ is a function of compact support and such that $\psi \equiv 1$ in $(0,4 \pi)$.

As a consequence of Lemma 8.4 we have:

$$
\begin{aligned}
\int_{0}^{4 \pi}\left|\sum_{n \in \mathbb{Z}} c_{n}\left(e^{i n t}-e^{i \sigma_{n}^{\epsilon} t}\right)\right|^{2} d t & =\int_{0}^{4 \pi}\left|\sum_{n \in \mathbb{Z}} c_{n} e^{i n t}\left(1-e^{i \lambda_{n, 0}^{\epsilon} t}\right)\right|^{2} \\
& \leq M^{2} a^{2} \sum_{n \in \mathbb{Z}}\left|c_{n}\right|^{2}
\end{aligned}
$$

and moreover

$$
\begin{aligned}
\int_{0}^{4 \pi} & \left|\sum_{n \in \mathbb{Z}} c_{n}\left(i t e^{i n t}-\frac{e^{i \sigma_{n}^{\varepsilon} t}-e^{i \zeta_{n}^{\varepsilon} t}}{\sigma_{n}^{\varepsilon}-\zeta_{n}^{\varepsilon}}\right)\right|^{2} d t \\
& =\int_{0}^{4}\left|\sum_{n \in \mathbb{Z}} c_{n} e^{i n t}\left(i t-\frac{e^{i \lambda_{n, 0}^{\varepsilon} t}-e^{i \lambda_{n, 1}^{\varepsilon} t}}{\lambda_{n, 0}^{\varepsilon}-\lambda_{n, 1}^{\varepsilon}}\right)\right|^{2} d t \leq M^{2} a^{2} \sum_{n \in \mathbb{Z}}\left|c_{n}\right|^{2}
\end{aligned}
$$

with $a>0$ depending on the constant $\delta>0$ of (8.10) and such that $a(\delta) \rightarrow$ 0 as $\delta \rightarrow 0$. On the other hand, the constant $M>0$ on (8.14) and (8.15) does not depend on $0<\varepsilon<1$ and $0<\delta<1$.

From (8.14) and (8.15) we deduce that

$$
\begin{aligned}
& \int_{0}^{4 \pi}\left|\sum_{n \in \mathbb{Z}} c_{n, 0}\left(e^{i n t}-e^{i \sigma_{n}^{\varepsilon} t}\right)+\sum_{n \in \mathbb{Z}} c_{n, 1}\left(i t e^{i n t}-\frac{e^{i \sigma_{n}^{\varepsilon} t}-e^{i \zeta_{n}^{\varepsilon} t}}{\sigma_{n}^{\varepsilon}-\zeta_{n}^{\varepsilon}}\right)\right|^{2} d t \\
& \quad \leq 2 M^{2} a^{2} \sum_{n \in \mathbb{Z}}\left(\left|c_{n, 0}\right|^{2}+\left|c_{n, 1}\right|^{2}\right) .
\end{aligned}
$$

We need now the following two results from D. Ulrich [12]:

LEMMA 8.5. The set $\left\{e^{i n t}, t e^{i n t}\right\}_{n \in \mathbb{Z}}$ forms a Riesz basis of the Hilbert space $L^{2}(0,4 \pi)$.

Lemma 8.6. Let $\left\{x_{n}\right\}$ be a Riesz basis of the Hilbert space $H$ such that

$$
A^{2} \sum_{n \in \mathbb{Z}}\left|a_{n}\right|^{2} \leq\left\|\sum_{n \in \mathbb{Z}} a_{n} x_{n}\right\|_{H}^{2} \leq B^{2} \sum_{n \in \mathbb{Z}}\left|a_{n}\right|^{2} .
$$

Assume that there exists $0<A_{1}<A$ and a set $\left\{y_{n}\right\} \subset H$ such that

$$
\left\|\sum_{n \in \mathbb{Z}} a_{n} y_{n}\right\|_{H}^{2} \leq A_{1}^{2} \sum_{n \in \mathbb{Z}}\left|a_{n}\right|^{2}
$$

for all $\left\{a_{n}\right\} \in \ell^{2}$. 
Then, $\left\{x_{n}+y_{n}\right\}$ forms a Riesz basis of $H$ and

$$
\left(A-A_{1}\right)^{2} \sum_{n \in \mathbb{Z}}\left|a_{n}\right|^{2} \leq\left\|\sum_{n \in \mathbb{Z}} a_{n}\left(x_{n}+y_{n}\right)\right\|_{H}^{2} \leq\left(B+A_{1}\right)^{2} \sum_{n \in \mathbb{Z}}\left|a_{n}\right|^{2} .
$$

In view of Lemma 8.5, $\left\{x_{n}\right\}=\left\{e^{i n t}, t e^{i n t}\right\}_{n \in \mathbb{Z}}$ form a Riesz basis of $L^{2}(0,4 \pi)$. Using (8.16), taking into account that $a(\delta) \rightarrow 0$ as $\delta \rightarrow 0$ and applying Lemma 8.6 with $\left\{x_{n}\right\}$ as above and $\left\{y_{n}\right\}=\left\{-e^{i \sigma_{n}^{\epsilon} t},-\left(\frac{e^{i \sigma_{n}^{\epsilon} t}-\epsilon^{i \zeta_{n}^{\epsilon} t}}{\sigma_{n}^{\epsilon}-\zeta_{n}^{\epsilon}}\right)\right\}$ we deduce that Theorem 8.3 holds provided $\delta>0$ is small enough.

For the applications we need a more general version of Theorem 8.3:

THEOREM 8.7. Let $\left\{\omega_{n}\right\}_{n \in \mathbb{Z}}$ be a sequence such that $\omega_{n} \neq n$ for all $n \in \mathbb{Z}$ and $\left|\omega_{n}-n\right| \rightarrow 0$ when $n \rightarrow \infty$. For any $0<\varepsilon<1$ we consider two sequences $\left\{\sigma_{n}^{\varepsilon}\right\}_{n \in \mathbb{Z}}$ and $\left\{\zeta_{n}^{\varepsilon}\right\}_{n \in \mathbb{Z}}$ of distinct real numbers such that $\sigma_{n}^{\varepsilon} \neq \zeta_{n}^{\varepsilon}$. Then, there exists $\delta>0$ such that, if

$$
\max \left\{\left|\omega_{n}-\sigma_{n}^{\varepsilon}\right|,\left|n-\zeta_{n}^{\varepsilon}\right|\right\}<\delta \text { for all } n \in \mathbb{Z} \text { and } 0<\varepsilon<1
$$

then the two conclusions of Theorem 8.3 hold.

Proof. Given $N \in \mathbb{N}$ large we replace $\left\{\sigma_{n}^{\varepsilon}\right\}$ by

$$
\tilde{\sigma}_{n}^{\varepsilon}= \begin{cases}n+\gamma_{n}^{\varepsilon} & |n| \leq N \\ \sigma_{n}^{\varepsilon} & |n|>N\end{cases}
$$

where $\left|\gamma_{n}^{\varepsilon}\right|<\delta, \tilde{\sigma}_{n}^{\varepsilon} \neq \zeta_{n}^{\varepsilon}$ and $\left\{\tilde{\sigma}_{n}^{\varepsilon}\right\}$ are all distinct.

If $\delta>0$ in (8.18) is small enough and $N$ is chosen large enough, then the sequences $\left\{\widetilde{\sigma}_{n}^{\varepsilon}\right\}$ and $\left\{\zeta_{n}^{\varepsilon}\right\}$ satisfy the hypotheses of Theorem 8.3.

Proceeding as in A. Haraux [5] it is easy to see that the terms corresponding to $|n| \leq N$ in the Riesz basis $\left\{e^{i \widetilde{\sigma}_{n}^{\varepsilon} t}\right\} \cup\left\{\frac{e^{i \tilde{\sigma}_{n}^{\epsilon} t}-e^{i \zeta_{n}^{\epsilon} t}}{\widetilde{\sigma}_{n}^{\varepsilon}-\zeta_{n}^{\epsilon}}\right\}$ can be substituted by $\left\{e^{i \sigma_{n}^{\epsilon} t}\right\} \cup\left\{\frac{e^{i \sigma_{n}^{\epsilon} t}-e^{i \epsilon} \zeta_{n}^{\epsilon}}{\sigma_{n}^{\epsilon}-\zeta_{n}^{\epsilon}}\right\}$ and still keep a Riesz basis with uniform constants in $(8.11)$.

\subsection{UNIFORM PARTial CONTROLLABILITY of THE APPROXimate SYSTEMS}

We consider now the approximate system (1.2) with a control $v=v^{\varepsilon}(t)$ acting on the extreme $x=1$ :

$$
\begin{cases}u_{t t}=u_{x x}, & \text { for }-1<x<-\varepsilon, t>0 \\ \frac{1}{2 \varepsilon} u_{t t}=u_{x x}, & \text { for }-\varepsilon<x<\varepsilon, t>0 \\ u_{t t}=u_{x x}, & \text { for } \varepsilon<x<1, t>0 \\ u\left( \pm \varepsilon^{-}, t\right)=u\left( \pm \varepsilon^{+}, t\right), & \text { for } t>0 \\ u_{x}\left( \pm \varepsilon^{-}, t\right)=u_{x}\left( \pm \varepsilon^{+}, t\right) & \text { for } t>0 \\ u(-1, t)=0, u(1, t)=v^{\varepsilon}(t), & \text { for } t>0 .\end{cases}
$$

Using HUM the controllability problem for (8.19) can be reduced to the obtention of suitable observability inequalities for the uncontrolled system (1.2). Since the density $\rho^{\varepsilon}(x)=1+\frac{1}{2 \varepsilon} \chi_{(-\varepsilon, \varepsilon)}(x)$ of system $(1.2)$ is in $B V$ it is easy to see that, for $T>4$, there exists a constant $C_{\varepsilon}>0$ such that

$$
E^{\varepsilon} \leq C_{\varepsilon} \int_{0}^{T}\left|u_{x}(1, t)\right|^{2} d t
$$


for any finite-energy solution of (1.2).

However, the constant $C_{\varepsilon} \rightarrow \infty$ as $\varepsilon \rightarrow 0$. This can be easily seen taking into account that the spectral gap vanishes as $\varepsilon \rightarrow 0$.

As a consequence of this, for any $T>4$ system (8.19) is exactly controllable in $L^{2}(-1,1) \times H^{-1}(-1,1)$ with $L^{2}(0, T)$-controls. However, there are not uniform bounds (with respect to $\varepsilon \rightarrow 0$ ) on the control $v^{\varepsilon}$ in $L^{2}(0, T)$ in terms of the norm of the controlled initial data.

The results of previous sections suggest that one can expect some uniform bounds in the observability inequalities if one introduces the finitedimensional asymmetric spaces.

Thus, let us consider initial data of the form

$$
U_{0}^{\varepsilon}=\sum_{|k| \leq K(\varepsilon)}\left(a_{k}^{\varepsilon} \bar{\phi}_{k}^{\varepsilon}+b_{k}^{\varepsilon} \bar{\psi}_{k}^{\varepsilon}\right)
$$

for the uncontrolled system (1.2) and let us denote by $H_{\varepsilon}$ the Hilbert space of the those initial data endowed with the norm

$$
\left\|U_{0}^{\varepsilon}\right\|_{\varepsilon}^{2}=\left|b_{-1}^{\varepsilon}\right|^{2}+\left|b_{1}^{\varepsilon}\right|^{2}+\sum_{|k| \leq K(\varepsilon)}\left(\delta_{k}^{\varepsilon}\right)^{2}\left|a_{k}^{\varepsilon}\right|^{2}+\sum_{\substack{|k| \leq K(\varepsilon) \\ k \neq \pm 1}}\left|a_{k-\sigma_{k}}^{\varepsilon}+b_{k}^{\varepsilon}\right|^{2},
$$

where, we recall, $K(\varepsilon) \sim \varepsilon^{-1 / 6}$.

Solutions $U^{\varepsilon}$ of (1.2) with initial data of the form (8.21) can be developed in Fourier series as follows:

$$
U^{\varepsilon}(t)=\sum_{|k| \leq K(\varepsilon)}\left(a_{k}^{\varepsilon} e^{i \alpha_{k}^{\varepsilon} t} \overline{\phi_{k}^{\varepsilon}}+b_{k}^{\varepsilon} e^{i \beta_{k}^{\varepsilon} t} \bar{\psi}_{k}^{\varepsilon}\right) .
$$

Clearly

$$
u_{x}^{\varepsilon}(1, t)=\sum_{|k| \leq K(\varepsilon)}\left(a_{k}^{\varepsilon} e^{i \alpha_{k}^{\varepsilon} t}\left(\phi_{k}^{\varepsilon}\right)^{\prime}(1)+b_{k}^{\varepsilon} e^{i \beta_{k}^{\varepsilon} t}\left(\psi_{k}^{\varepsilon}\right)^{\prime}(1)\right) .
$$

The following uniform observability result holds:

Theorem 8.8. Assume that $T>4$. Then, there exists $C>0$ such that

$$
\frac{1}{C} \int_{0}^{T}\left|u_{x}^{\varepsilon}(1, t)\right|^{2} d t \leq\left\|U_{0}^{\varepsilon}\right\|_{\varepsilon}^{2} \leq C \int_{0}^{T}\left|u_{x}^{\varepsilon}(1, t)\right|^{2} d t
$$

holds for any solution of (1.2) with initial data $U_{\varepsilon}^{0} \in H_{\varepsilon}$ and for every $0<\varepsilon<\varepsilon_{0}$ with $\varepsilon_{0}>0$ small enough.

REMARK 8.9. We do not know whether uniform estimates of the form (8.25) may be true for all initial data $U_{0}$ without the restriction on the number of non-zero Fourier coefficients of being less than $K(\varepsilon)$. The present state of the theory of non-harmonic Fourier series does not seem to allow to give an answer to this question. Notice that in the norm $\|\cdot\|_{\varepsilon}$ each Fourier coefficient has been weighted by the distance between the corresponding consecutive eigenvalues. This enables to find counterexamples for such an uniform estimate and the situation seems much more delicate than when analyzing the classical inequalities of the form (8.20). 
Proof. In view of (8.24) we can rewrite $u_{x}^{\varepsilon}(1, t)$ as follows:

$$
\begin{aligned}
u_{x}^{\varepsilon}(1, t)= & \sum_{|k| \leq K(\varepsilon)}\left[a_{k}^{\varepsilon}\left(\phi_{k}^{\varepsilon}\right)^{\prime}(1)\left(e^{i \alpha_{k}^{\varepsilon} t}-e^{i \beta_{k}^{\varepsilon} t}\right)\right. \\
& \left.+\left(a_{k}^{\varepsilon}\left(\phi_{k}^{\varepsilon}\right)^{\prime}(1)-b_{k}^{\varepsilon}\left(\psi_{k}^{\varepsilon}\right)^{\prime}(1)\right) e^{i \beta_{k}^{\varepsilon} t}\right] .
\end{aligned}
$$

Using the asymptotic estimates of Theorem 4.7 on the eigenvalues of system (1.2) and Theorem 8.7 we deduce that if $K(\varepsilon)=\delta \varepsilon^{-1 / 6}$ with $\delta>0$ small enough and $0<\varepsilon<\varepsilon_{0}$ with $\varepsilon_{0}>0$ sufficiently small, then

$$
\begin{aligned}
& \frac{1}{C} \sum_{|k| \leq K(\varepsilon)}\left[\left(\delta_{k}^{\varepsilon}\right)^{2}\left|a_{k}^{\varepsilon}\right|^{2}\left|\left(\phi_{k}^{\varepsilon}\right)^{\prime}(1)\right|^{2}+\left|a_{k}^{\varepsilon}\left(\phi_{k}^{\varepsilon}\right)^{\prime}(1)-b_{k}^{\varepsilon}\left(\psi_{k}^{\varepsilon}\right)^{\prime}(1)\right|^{2}\right] \\
& \quad \leq \int_{0}^{T}\left|u_{x}^{\varepsilon}(1, t)\right|^{2} d t \\
& \quad \leq C \sum_{|k| \leq K(\varepsilon)}\left[\left(\delta_{k}^{\varepsilon}\right)^{2}\left|a_{k}^{\varepsilon}\right|^{2}\left|\left(\phi_{k}^{\varepsilon}\right)^{\prime}(1)\right|^{2}+\left|a_{k}^{\varepsilon}\left(\phi_{k}^{\varepsilon}\right)^{\prime}(1)-b_{k}^{\varepsilon}\left(\psi_{k}^{\varepsilon}\right)^{\prime}(1)\right|^{2}\right] .
\end{aligned}
$$

On the other hand, it is easy to see that

$$
\left|\left(\phi_{k}^{\varepsilon}\right)^{\prime}(1)+1\right| \leq C \sqrt{\varepsilon} \quad, \quad \text { if }|k| \leq K(\varepsilon)
$$

and

$$
\left|\left(\phi_{k}^{\varepsilon}\right)^{\prime}(1)-\left(\psi_{k}^{\varepsilon}\right)^{\prime}(1)\right| \leq \frac{\delta_{k}^{\varepsilon}}{2} \quad, \quad \text { if } k_{0} \leq|k| \leq K(\varepsilon)
$$

for $k_{0}>0$ large enough independent of $\varepsilon$.

Therefore, (8.25) is an easy consequence of (8.27).

Let us consider now the controlled system (8.19) with initial data in $L^{2}(-1,1) \times H^{-1}(-1,1)$. For any $\varepsilon>0$ we define the projection $\pi_{\varepsilon}$ from this space into the finite-dimensional subspace of elements of the form (8.21).

As a consequence of the uniform observability result of Theorem 8.8 and applying HUM (see [7]) the following uniform partial controllability result holds:

Theorem 8.10. Assume that $T>4$ and $0<\varepsilon<\varepsilon_{0}$. For any $\left(u_{0}, u_{1}\right) \in$ $L^{2}(-1,1) \times H^{-1}(-1,1)$ there exists a control $v_{\varepsilon} \in L^{2}(0, T)$ such that the solution $u^{\varepsilon}$ of (8.19) taking the initial data

$$
u^{\varepsilon}(0)=u_{0}, \quad\left(u^{\varepsilon}\right)^{\prime}(0)=u_{1} \text { in }(-1,1)
$$

satisfies the final conditions

$$
\pi_{\varepsilon}\left(u^{\varepsilon}(T),\left(u^{\varepsilon}\right)^{\prime}(T)\right)=0 .
$$

Moreover, there exists a constant $C>0$ independent of $0<\varepsilon<\varepsilon_{0}$ such that

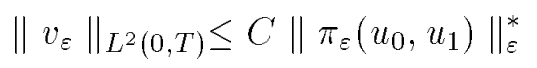

and

$$
\max _{0 \leq t \leq T}\left\|\pi_{\varepsilon}\left(u^{\varepsilon}(t),\left(u^{\varepsilon}\right)^{\prime}(t)\right)\right\|_{\varepsilon}^{*} \leq C\left\|v_{\varepsilon}\right\|_{L^{2}(0, T)}
$$

where $\|\cdot\|_{\varepsilon}^{*}$ is the dual norm with respect to $\|\cdot\|_{\varepsilon}$.

Esaim: CoCv, July 1997, Vol.2, PP. 231-280 
REMARK 8.11. If $U_{0}^{\varepsilon}$ is of the form (8.21) the norm $\|\cdot\|_{\varepsilon}^{*}$ is defined as follows:

$$
\left\|U_{0}^{\varepsilon}\right\|_{\varepsilon}^{*}=\left[\left|b_{-1}^{\varepsilon}\right|^{2}+\left|b_{1}^{\varepsilon}\right|^{2}+\sum_{|k| \leq K(\varepsilon)}\left(\delta_{k}^{\varepsilon}\right)^{2}\left|a_{k}\right|^{2}+\sum_{\substack{|k| \leq K(\varepsilon) \\ k \neq \pm 1}}\left|a_{k-\sigma_{k}}^{\varepsilon}+b_{k}^{\varepsilon}\right|^{2}\right]^{1 / 2} .
$$

Theorem 8.10 states that we can control uniformly the first $K(\varepsilon)$ frequencies of solutions of the approximated system (8.19). Uniformly means that, in particular, when the initial data are fixed the control is bounded in $L^{2}(0, T)$ as $\varepsilon \rightarrow 0$ and, on the other hand, that the projection of the solutions $u^{\varepsilon}$ into the finite-dimensional subspace of elements with $K(\varepsilon)$ noninitial Fourier coefficients remains bounded for all $0 \leq t \leq T$ as $\varepsilon \rightarrow 0$.

We do not give the details of the proof of this Theorem which is a straightforward consequence of Theorem 8.8. When passing to the limit in Theorem 8.12 we will briefly sketch the proof of Theorem 8.10 too.

\subsection{Passage to the limit}

We are now in conditions to obtain the controllability result of Theorem 8.1 as the limit when $\varepsilon \rightarrow 0$ of the uniform, partial controllability results of the section 8.3 above:

Theorem 8.12. Assume that $T>4$ and $0<\varepsilon<\varepsilon_{0}$. Let $\left(u_{0}, u_{1}, z_{0}, z_{1}\right)$ be as in (8.4) an element of the infinite-dimensional asymmetric space $H_{-1}$ in (8.7), with Fourier coefficients $\left\{a_{k}\right\} \cup\left\{b_{k}\right\}$. On the other hand, let $v \in$ $L^{2}(0, T)$ be the control obtained in Theorem 8.1 for the limit system (8.1) and $u$ the corresponding solution.

Now let $U_{0}^{\varepsilon}$ be the truncated data

$$
U_{0}^{\varepsilon}=\sum_{|k| \leq K(\varepsilon)}\left[a_{k} \bar{\phi}_{k}^{\varepsilon}+b_{k} \bar{\psi}_{k}^{\varepsilon}\right]
$$

and $v^{\varepsilon} \in L^{2}(0, T)$ the corresponding partial controls of Theorem 8.10.

Then,

$$
\left\{\begin{array}{l}
v^{\varepsilon} \rightarrow v \text { weakly in } L^{2}(0, T) \\
\pi_{\varepsilon}\left(u^{\varepsilon}(t), u_{t}^{\varepsilon}(t)\right) \rightarrow\left(u(t), u_{t}(t)\right) \text { weakly } * \text { in } L^{\infty}\left(0, T ; H_{-1}\right) .
\end{array}\right.
$$

REMARK 8.13. This result, roughly, establishes the continuity of the controls as $\varepsilon \rightarrow 0$. Of course, the controls $v^{\varepsilon}$ and $v$ are not unique and therefore, the meaning of (8.34) has to be made precise.

Even if the controls ar not unique, HUM provides a unique choice of them. In (8.34) we state the convergence of these controls obtained by HUM.

Convergences (8.34) can be improved. Indeed, it is not hard to see that the controls converge strongly in $L^{2}(0, T)$ and that the solutions are such that the second convergence of $(8.34)$ holds strongly in $L^{p}\left(0, T ; H_{-1}\right)$ for any $1 \leq p<\infty$.

Sketch of proof. We will not give all the details of the proof that follows by standard arguments (see for instance [7], [1] and [2] for similar convergence results in other contexts). 
The control $v^{\varepsilon}$ given by HUM for the approximate system can be characterized in the following way.

We solve the uncontrolled system

$$
\left\{\begin{array}{l}
\rho^{\varepsilon}(x) \varphi_{t t}^{\varepsilon}-\varphi_{x x}^{\varepsilon}=0, \quad-1<x<1, \quad 0<t<T \\
\varphi^{\varepsilon}(-1, t)=\varphi^{\varepsilon}(1, t)=0, \quad 0<t<T \\
\varphi^{\varepsilon}(x, 0)=\varphi_{0}^{\varepsilon}(x), \varphi_{t}^{\varepsilon}(x, 0)=\varphi_{1}^{\varepsilon}(x), \quad-1<x<1
\end{array}\right.
$$

with $\rho^{\varepsilon}(x)=1+\frac{1}{2 \varepsilon} \chi_{(-\varepsilon, \varepsilon)}(x)$ and initial data $\left(\varphi_{0}^{\varepsilon}, \varphi_{1}^{\varepsilon}\right)$ of the form

$$
\left(\varphi_{0}^{\varepsilon}, \varphi_{1}^{\varepsilon}\right)=\sum_{|k| \leq K(\varepsilon)}\left(c_{k}^{\varepsilon} \bar{\phi}_{k}^{\varepsilon}+d_{k}^{\varepsilon} \bar{\psi}_{k}^{\varepsilon}\right) .
$$

Given an initial data $U_{0}^{\varepsilon}$ of the form (8.33) we define the quadratic functional:

$$
\begin{aligned}
J_{\varepsilon}\left(\varphi_{0}^{\varepsilon}, \varphi_{1}^{\varepsilon}\right)= & \frac{1}{2} \int_{0}^{T}\left|\varphi_{x}^{\varepsilon}(1, t)\right|^{2} d t-b_{-1} \bar{d}_{-1}^{\varepsilon}-b_{1} \bar{d}_{1}^{\varepsilon} \\
& -\sum_{\substack{|k| \leq K(\varepsilon) \\
k \neq \pm 1}}\left[\delta_{k-\sigma_{k}}^{\varepsilon}\left(a_{k-\sigma_{k}}-b_{k}\right)\left(\bar{c}_{k-\sigma_{k}}^{\varepsilon}-\bar{d}_{k}^{\varepsilon}\right)\right. \\
& \left.+\delta_{k-\sigma_{k}}^{\varepsilon}\left(a_{k-\sigma_{k}}+b_{k}\right)\left(\bar{c}_{k-\sigma_{k}}^{\varepsilon}+\bar{d}_{k}^{\varepsilon}\right)\right] .
\end{aligned}
$$

Clearly $J_{\varepsilon}$ is continuous from the space $H_{\varepsilon}$ of initial data of the form (8.36) into $\mathbb{R}$. Notice that in (8.37), the last two terms denotes the duality between the data $\left(\varphi_{0}^{\varepsilon}, \varphi_{1}^{\varepsilon}\right)$ and the data $U_{0}^{\varepsilon}$ of the controlled system.

In view of Theorem 8.8 it is easy to see that $J_{\varepsilon}$ is uniformly coercive. More precisely, there exists $C>0$ such that

$$
J_{\varepsilon}\left(\varphi_{0}^{\varepsilon}, \varphi_{1}^{\varepsilon}\right) \geq C\left\|\left(\varphi_{0}^{\varepsilon}, \varphi_{1}^{\varepsilon}\right)\right\|_{\varepsilon}^{2}-\frac{1}{C}, \quad \forall 0<\varepsilon<\varepsilon_{0}, \quad \forall\left(\varphi_{0}^{\varepsilon}, \varphi_{1}^{\varepsilon}\right) \in H_{\varepsilon} .
$$

Therefore, the minimizers $\left(\varphi_{0}^{\varepsilon}, \varphi_{1}^{\varepsilon}\right)$ for $J_{\varepsilon}$ in $H_{\varepsilon}$ exist for each $0<\varepsilon<\varepsilon_{0}$, are unique and uniformly bounded in the sense that the quantity $\left\|\left(\varphi_{0}^{\varepsilon}, \varphi_{1}^{\varepsilon}\right)\right\|_{\varepsilon}$ remains bounded.

Note that, in order to obtain (8.38), we have used the fact that the data $\left(u_{0}, u_{1}, z_{0}, z_{1}\right)$ belong to $H_{-1}$ in an essential way.

The minimizer $\left(\varphi_{0}^{\varepsilon}, \varphi_{1}^{\varepsilon}\right)$ of $J_{\varepsilon}$ in $H_{\varepsilon}$ is characterized by

$$
\begin{gathered}
\int_{0}^{T} \varphi_{x}^{\varepsilon}(1, t) \psi_{x}^{\varepsilon}(1, t) d t=b_{-1} \overline{\eta_{-1}^{\varepsilon}}+b_{1} \overline{\eta_{1}^{\varepsilon}} \\
+\sum_{\substack{|k| \leq K(\varepsilon) \\
k \neq \pm 1}}\left[\delta_{k-\sigma_{k}}^{\varepsilon}\left(a_{k-\sigma_{k}}-b_{k}\right)\left(\overline{\xi_{k-\sigma_{k}}^{\varepsilon}}-\overline{\eta_{k}^{\varepsilon}}\right)\right. \\
\left.\quad+\delta_{k-\sigma_{k}}^{\varepsilon}\left(a_{k-\sigma_{k}}+b_{k}\right)\left(\overline{\bar{\xi}_{k-\sigma_{k}}^{\varepsilon}}+\overline{\eta_{k}^{\varepsilon}}\right)\right]
\end{gathered}
$$

for any solution $\psi^{\varepsilon}$ of (8.35) with initial data in $H_{\varepsilon}$ and Fourier coefficents $\left\{\xi_{k}^{\varepsilon}\right\} \cup\left\{\eta_{k}^{\varepsilon}\right\}$.

The control $v^{\varepsilon}$ for the approximate system is precisely

$$
v^{\varepsilon}=\varphi_{x}^{\varepsilon}(1, t)
$$

and it is uniformly bounded in $L^{2}(0, T)$ in view of Theorem 8.8 .

Esaim: CoCv, July 1997, Vol.2, PP. 231-280 
In what concerns the limit system (8.1), the control $v$ is also of the form

$$
v(t)=\varphi_{x}(1, t)
$$

where $\varphi$ is a solution of the adjoint system

$$
\left\{\begin{array}{lll}
\varphi_{t t}=\varphi_{x x}, & -1<x<0, & 0<t<T \\
\varphi_{t t}=\varphi_{x x}, & 0<x<1, & 0<t<T \\
\varphi\left(0^{+}, t\right)=\varphi\left(0^{-}, t\right)=\zeta(t), & & 0<t<T \\
M \zeta_{t t}(t)=\varphi_{x}\left(0^{+}, t\right)-\varphi_{x}\left(0^{-}, t\right), & 0<t<T \\
\varphi(-1, t)=\varphi(1, t)=0, & 0<t<T
\end{array}\right.
$$

with initial data $\phi_{0}$ of the form

$$
\phi_{0}=\sum_{k \in \mathbb{Z}}\left[a_{k} \bar{\phi}_{k}+d_{k} \bar{\psi}_{k}\right]
$$

belonging to the asymmetric space $H$ of (5.9).

The initial data corresponding to the solution $\varphi$ that determine the control $v$ by $(8.41)$ are obtained by minimizing the quadratic functional

$$
\begin{aligned}
& J\left(\phi_{0}\right)=\frac{1}{2} \int_{0}^{T}\left|\varphi_{x}(1, t)\right|^{2} d t-b_{-1} \overline{d_{-1}}-b_{1} \overline{d_{1}} \\
& -\sum_{k \in \mathbb{Z} \backslash\{0, \pm 1\}}\left[\delta_{k-\sigma_{k}}\left(a_{k-\sigma_{k}}-b_{k}\right)\left(\bar{c}_{k-\sigma_{k}}-\bar{d}_{k}\right)\right. \\
& \left.\quad+\delta_{k-\sigma_{k}}\left(a_{k-\sigma_{k}}+b_{k}\right)\left(\bar{c}_{k-\sigma_{k}}+\bar{d}_{k}\right)\right]
\end{aligned}
$$

over $H$. The minimum is characterized by

$$
\begin{aligned}
& \int_{0}^{T} \varphi_{x}(1, t) \psi_{x}(1, t) d t \\
& =b_{-1} \overline{\eta_{-1}}+b_{1} \overline{\eta_{1}}+\sum_{k \in \mathbb{Z} \backslash\{0, \pm 1\}}\left[\delta_{k-\sigma_{k}}\left(a_{k-\sigma_{k}}-b_{k}\right)\left(\overline{\xi_{k-\sigma_{k}}}-\overline{\eta_{k}}\right)\right. \\
& \left.\quad+\delta_{k-\sigma_{k}}\left(a_{k-\sigma_{k}}+b_{k}\right)\left(\overline{\xi_{k-\sigma_{k}}}+\overline{\eta_{k}}\right)\right]
\end{aligned}
$$

for all solution $\psi$ of $(8.42)$ with data in $H$ corresponding to Fourier coefficients $\left\{\xi_{k}\right\} \cup\left\{\eta_{k}\right\}$.

Using the characterizations (8.39) and (8.45) it is easy to see the weak limits of subsequences of $v_{\varepsilon}$ solve $(8.45)$. The solution of $(8.45)$ being unique this allows to conclude the first part of (8.34).

Applying the identities (8.39) and (8.45) with $\psi^{\varepsilon}=\varphi^{\varepsilon}$ and $\psi=\varphi$ respectively and using the convergence of the right hand side terms (they are linear on $\varphi^{\varepsilon}$ and $\varphi$ and therefore converge by continuity with respect to the weak topologies) one deduces that

$$
\int_{0}^{T}\left|\varphi_{x}^{\varepsilon}(1, t)\right|^{2} d t \rightarrow \int_{0}^{T}\left|\varphi_{x}(1, t)\right|^{2} d t
$$

and concludes the strong convergence of the controls.

Finally, using a transposition argument, it is easy to see that the solutions of the controlled systems converge in the sense of the second statement of (8.34). 


\section{RefERENCES}

[1] D. Cioranescu, P. Donato, E. Zuazua: Exact boundary controllability for the wave equation in domains with small holes, J. Math. Pures et Appl., 71, 1992, 343-377.

[2] D. Cioranescu, P. Donato, E. Zuazua: Strong convergence on the exact controllability of the wave equation in perforated domains, in Control of Distributed Parameter Systems, Desch, Kappel and Kunisch eds., Birkhaüser-Verlag, INMS Series, 100, 1991, 99-113.

[3] Yu. D. Galovaty, S. A. Nazarov, O. A. Oleinik, T. S. Soboleva: Eigenoscillations of a string with additional mass, Siberian Math. J., 29 (5), 1988, 744-760.

[4] S. Hansen, E. Zuazua: Exact controllability and stabilization of a vibrating string with an interior point mass, SIAM J. Cont. Optim., 33 (5), 1995, 1357-1391.

[5] A. Haraux: Séries lacunaires et contrôle semi-interne des vibrations d'une plaque rectangulaire, J. Math. Pures et Appl., 68, 1989, 457-465.

[6] J. L. Lions: Contrôlabilité exacte, stabilisation et perturbations de systèmes distribués. Tome 1. Contrôlabilité exacte, Masson, RMA8, 1988.

[7] J. L. Lions: Contrôlabilité exacte, stabilisation et perturbations de systèmes distribués. Tome 2, Perturbations, Masson, RMA9, 1988.

[8] A. McNabb, R. S. Anderssen, E. R. Lapwood: Asymptotic behavior of the eigenvalues of a Sturm-Liouville system with discontinuous coefficients, J. Math. Anal. Applications, 54, 1976, 741-751.

[9] O. A. Oleinick, A. S. Shamaev, B. A. Yosifian: Mathematical Problems in Elasticity and Homogenization, North-Holland, 1992.

[10] J. Poschel, E. Trubowitz: Inverse spectral theory, Academic Press, 1986.

[11] E. Sánchez-Palencia: Nonhomogeneous Media and Vibrating Theory, Lecture Notes in Physics 127, Springer-Verlag, 1980.

[12] D. Ulrich: Divided differences and systems of nonharmonic Fourier series, Proc. AMS, 80 (1), 1980, 47-57. 